Draft VERSiOn July 22, 2018

Preprint typeset using $\mathrm{LAT}_{\mathrm{E}} \mathrm{X}$ style emulateapj v. 08/22/09

\title{
XMM-NEWTON AND SUZAKU X-RAY SHADOWING MEASUREMENTS OF THE SOLAR WIND CHARGE EXCHANGE, LOCAL BUBBLE, AND GALACTIC HALO EMISSION
}

\author{
David B. Henley and Robin L. Shelton \\ Department of Physics and Astronomy, University of Georgia, Athens, GA 30602; dbh@physast.uga.edu, rls@physast.uga.edu \\ Draft version July 22, 2018
}

\begin{abstract}
We present results from a sample of XMM-Newton and Suzaku observations of interstellar clouds that cast shadows in the soft X-ray background (SXRB) - the first uniform analysis of such a sample from these missions. By fitting to the on- and off-shadow spectra, we separated the foreground and Galactic halo components of the SXRB. We tested different foreground models - two solar wind charge exchange (SWCX) models and a Local Bubble (LB) model. We also examined different abundance tables. We found that Anders \& Grevesse (1989) abundances, commonly used in previous SXRB studies, may result in overestimated foreground brightnesses and halo temperatures. We also found that assuming a single solar wind ionization temperature for a SWCX model can lead to unreliable results. We compared our measurements of the foreground emission with predictions of the SWCX emission from a smooth solar wind, finding only partial agreement. Using available observation-specific SWCX predictions and various plausible assumptions, we placed an upper limit on the LB's O VII intensity of $\sim 0.8$ photons $\mathrm{cm}^{-2} \mathrm{~s}^{-1} \mathrm{sr}^{-1}$ (90\% confidence). Comparing the halo results obtained with SWCX and LB foreground models implies that, if the foreground is dominated by SWCX and is brighter than $\sim 1.5 \times 10^{-12} \mathrm{erg} \mathrm{cm}^{-2} \mathrm{~s}^{-1} \mathrm{deg}^{-2}(0.4-1.0 \mathrm{keV})$, then using an LB foreground model may bias the halo temperature upward and the $0.5-2.0 \mathrm{keV}$ surface brightness downward by $\sim(0.2-0.3) \times$ $10^{6} \mathrm{~K}$ and $\sim(1-2) \times 10^{-12} \mathrm{erg} \mathrm{cm}^{-2} \mathrm{~s}^{-1} \mathrm{deg}^{-2}$, respectively. Similarly, comparing results from different observatories implies that there may be uncertainties in the halo temperature and surface brightness of up to $\sim 0.2 \times 10^{6} \mathrm{~K}$ and $\sim 25 \%$, respectively, in addition to the statistical uncertainties. These uncertainties or biases may limit the ability of X-ray measurements to discriminate between Galactic halo models.

Subject headings: Galaxy: halo - ISM: general - ISM: individual (Local Bubble) - solar wind - X-rays: diffuse background - X-rays: ISM
\end{abstract}

\section{INTRODUCTION}

Several emission components contribute to the diffuse soft X-ray background (SXRB) emission that is observed in all directions on the sky (McCammon \& Sanders 1990). The closest, and most recently discovered, source is solar wind charge exchange (SWCX) emission, which arises from charge exchange (CX) reactions between solar wind ions and neutral $\mathrm{H}$ and $\mathrm{He}$ in the heliosphere or the Earth's exosphere (Cravens 2000; Robertson \& Cravens 2003a, b; Koutroumpa et al. 2006). Beyond that, there may be emission from the Local Bubble (LB), a cavity in the local interstellar medium (ISM) thought to be filled with $\sim 1 \times 10^{6} \mathrm{~K}$ gas (Sanders et al. 1977; Snowden et al. 1990; although see also Welsh \& Shelton 2009). Further out still, beyond the main Galactic disk, there is emission from $\sim(1-3) \times 10^{6} \mathrm{~K}$ gas in the Galactic halo (Burrows \& Mendenhall 1991; Wang \& Yu 1995; Kuntz \& Snowden 2000; Yoshino et al. 2009, hereafter Yosh09; Henley \& Shelton 2013, hereafter HS13) - this gas is also observed via X-ray absorption lines (e.g., Nicastro et al. 2002; Rasmussen et al. 2003; Yao \& Wang 2007; Gupta et al. 2012; Miller \& Bregman 2013). The most distant contributor to the SXRB is the extragalactic background of unresolved active galactic nuclei (AGN; e.g., Brandt \& Hasinger 2005).

In order to test models for the local (SWCX and/or $\mathrm{LB}$ ) and halo contributions to the SXRB, it is necessary to separate their emission. ${ }^{1}$ To this end, one can use observations of interstellar clouds that cast shadows in the SXRB by partially absorbing the distant emission (Burrows \& Mendenhall 1991; Snowden et al. 1991). By comparing the X-ray emission observed toward and to the side of such a shadow, one can determine the contributions to the emission from the foreground and from the hot halo.

Such shadowing observations were first carried out with the low-spectral-resolution proportional counters on board ROSAT (Burrows \& Mendenhall 1991; Snowden et al. 1991, 1993; Wang \& Yu 1995; Kuntz et al. 1997; Snowden et al. 2000). More recently, the CCD spectrometers on board XMM-Newton and Suzaku have been used for such observations (Smith et al. 2007; Galeazzi et al. 2007; Henley et al. 2007; Henlev \& Shelton 2008; Gupta et al. 2009; Lei et al. 2009; Henlev et al. 2015a). These instruments have higher spectral resolution, allowing some line emission features (e.g., O VII and O VIII) to be resolved in the spectra. However, the CCD data are only usable above $\sim 0.3$ or $\sim 0.4 \mathrm{keV}$, whereas ROSAT could observe in the so-called $1 / 4 \mathrm{keV}$ band $(\sim 0.1-0.284 \mathrm{keV})$. This means fewer shadowing targets are available to $X M M$ -

\footnotetext{
${ }^{1}$ Dealing with the extragalactic background component of the SXRB is relatively simple, as its spectrum is well characterized as a power law (e.g., Chen et al. 1997), albeit with evidence of steepening below $\sim 1 \mathrm{keV}$ (Roberts \& Warwick 2001).
} 
Newton and Suzaku - as the photoelectric absorption cross section decreases with increasing photon energy, fewer clouds are sufficiently optically thick in the XMM-Newton/Suzaku band that the contrast between the on- and off-cloud spectra allows one to separate the foreground and halo emission.

The shadowing clouds that have been observed with $X M M-N e w t o n$ and/or Suzaku have typically yielded halo temperatures and emission measures of $\sim 2 \times 10^{6} \mathrm{~K}$ and a few times $10^{-3} \mathrm{~cm}^{-6} \mathrm{pc}$, respectively (Smith et al. 2007; Galeazzi et al. 2007; Gupta et al. 2009; Lei et al. 2009; Henley et al. 2015a). However, these observations were not analyzed in a homogeneous fashion. In addition, the sensitivity of the halo results to the assumed foreground model was not tested. Furthermore, some of these studies used older abundance tables (e.g., Anders \& Grevesse 1989), in which the carbon, nitrogen, and oxygen abundances are now known to be too high for the sun and the local interstellar medium Asplund et al. 2009; Wilms et al. 2000). Using these older abundances may bias the results (see Section 4.2).

Here, we analyze as large a set as possible of XMMNewton and Suzaku shadowing observations in a uniform fashion. We test different foreground models in order to examine the sensitivity of the halo results to the choice of foreground model. Recent studies have argued that a combination of SWCX and LB emission is needed to explain the foreground emission in the $1 / 4 \mathrm{keV}$ (Smith et al. 2014; Galeazzi et al. 2014) and XMM-Newton/Suzaku (Koutroumpa et al. 2011) bands. However, the relative contributions of the LB and of SWCX to the foreground in an arbitrary XMM-Newton or Suzaku observation are not known. Therefore, we follow Henley et al. (2015a), and consider limits in which either LB or SWCX emission dominate the foreground. (Note that in most previous shadowing studies, an LBlike spectral model was used for the foreground.) We also examine the sensitivity of our results to the choice of abundance table used in the spectral analysis.

The remainder of the paper is arranged as follows. In Section 2 we describe our observation selection and data reduction, and we describe our spectral model in Section 3. We present our results in Section 44-in particular, we examine the results obtained with different foreground models (Section 4.1) and with different abundance tables (Section 4.2). We also compare our results with those from previously published shadowing analyses (Section 4.3). We discuss our results in Section [5] We first discuss which of the foreground models we examined is our preferred model, and why (Section 5.1). We then compare our foreground measurements with heliospheric SWCX model predictions (Section 5.2). We also use our foreground measurements to place limits on the emission from the LB (Section 5.3). Finally, we discuss our halo measurements in Section 5.4, including discussing possible uncertainties or biases that may arise from uncertainties in the foreground emission and from other aspects of the spectral modeling (Section 5.4.2), and discussing the use of such halo measurements in testing Galactic halo models (Section 5.4.3). We conclude with a summary in Section 6.

\section{OBSERVATION SELECTION AND DATA REDUCTION}

Our sample includes, but is not limited to, all previously published XMM-Newton and Suzaku shadowing observations that consist of separate on- and off-shadow pointings. The targets of these observations are MBM 12 (observed with Suzaku; Smith et al. 2007), MBM 20 (observed with XMM-Newton and Suzaku; Galeazzi et al. 2007; Gupta et al. 2009), and an unnamed dusty filament in the southern Galactic hemisphere, hereafter dubbed "filament" (observed with XMM-Newton and Suzaku; Henley et al. 2007; Henley \& Shelton 2008; Lei et al. 2009). Although MBM 12 has also been observed with XMM-Newton (Koutroumpa et al. 2011), we did not include these data in our sample because there is not a corresponding blank-sky off-cloud pointing. Furthermore, we have previously found that the on-MBM 12 observation analyzed by Koutroumpa et al. (2011) was too badly contaminated by soft protons to be included in our $X M M-N e w t o n$ survey of the SXRB Henley \& Shelton 2012).

To the above set of observations, we added a pair of unpublished Suzaku observations of MBM 16 (preliminary results from these observations have been presented by Ursino et al. 2014). Our sample also includes previously unpublished observations of two clouds in the northern Galactic hemisphere, which we refer to as G048+37 (observed with XMM-Newton) and G236+38 (observed with Suzaku). These clouds were identified as viable shadowing targets from the DIRBE-corrected IRAS maps of the diffuse $100-\mu \mathrm{m}$ intensity, $I_{100}$ (Schlegel et al. 1998). In particular, we sought pairs of directions within $\approx 3^{\circ}$ of each other with absorbing column densities, $N_{\mathrm{H}}$, of $>10 \times 10^{20}$ and $\lesssim 2 \times 10^{20} \mathrm{~cm}^{-2}$ (from the Snowden et al. $2000 I_{100}$-to- $N_{\mathrm{H}}$ conversion relation). With such a column density contrast we expected to be able to adequately separate the foreground and halo emission with reasonable-length pointings. These observations are the first published CCD-resolution shadowing observations from the northern Galactic hemisphere.

Another potential target in the northern hemisphere is MBM $36((l, b)=(4.2,+35.8))$, for which a pair of on- and off-cloud observations is available in the Suzaku archive (ObsIDs 508079020 and 508074010, respectively). However, this cloud lies in the direction of Scorpius-Centaurus (Sco-Cen) superbubble (Egger \& Aschenbach 1995), the X-ray emission from which would complicate the analysis of the SXRB spectra, making it difficult to isolate the foreground and halo components. We therefore excluded MBM 36 from our sample.

The details of our XMM-Newton and Suzaku shadowing observations are shown in Table 1 Figure 1 shows the locations of these shadows on the sky. This figure also shows the location of shadow G225.60-66.40 (G225-66 hereafter), analyzed by Henley et al. (2015a). Unlike the shadows analyzed here, the on- and off-shadow spectra for G225-66 were extracted from a single XMM-Newton field. However, the subsequent analysis of the spectra was essentially the same as that employed here (Section 31). We will include the results from G225-66 in our discussion (Section 5). 100-micron maps of the shadowing clouds are shown in Figure 2, The following two subsections describe the data reduction for the $X M M$ - 
Table 1

Observation Details

\begin{tabular}{|c|c|c|c|c|c|c|c|c|c|}
\hline $\begin{array}{c}\text { Shadow } \\
\text { (1) }\end{array}$ & $\begin{array}{c}\text { Observatory } \\
(2)\end{array}$ & $\begin{array}{l}\text { On or } \\
\text { off } \\
(3)\end{array}$ & $\begin{array}{l}\text { ObsID } \\
\text { (4) }\end{array}$ & $\begin{array}{c}\begin{array}{c}\text { Start } \\
\text { date }\end{array} \\
(5)\end{array}$ & $\begin{array}{c}l \\
(\mathrm{deg}) \\
(6)\end{array}$ & $\begin{array}{c}b \\
(\mathrm{deg}) \\
(7)\end{array}$ & $\begin{array}{c}\text { Nominal } \\
\text { exposure } \\
(\mathrm{ks}) \\
(8)\end{array}$ & $\begin{array}{c}\text { Usable } \\
\text { exposure } \\
(\mathrm{ks}) \\
(9)\end{array}$ & $\begin{array}{c}N_{\mathrm{H}} \\
\left(10^{20} \mathrm{~cm}^{-2}\right) \\
(10)\end{array}$ \\
\hline \multirow[t]{2}{*}{$\mathrm{G} 048+37$} & \multirow[t]{2}{*}{ XMM-Newton } & On & 0670900501 & 2012-02-01 & 47.74 & +37.28 & 33.0 & $19.9,21.6,12.8$ & 9.3 \\
\hline & & Off & 0670900201 & 2012-01-30 & 48.97 & +40.38 & 33.0 & $19.1,19.4,10.8$ & 2.2 \\
\hline \multirow{2}{*}{ MBM 12} & \multirow[t]{2}{*}{ Suzaku } & On & 500015010 & 2006-02-03 & 159.21 & -34.47 & 102.9 & 68.6 & 32.2 \\
\hline & & Off & 501104010 & $2006-02-06$ & 157.36 & -36.82 & 75.3 & 51.1 & 3.8 \\
\hline \multirow{2}{*}{ MBM 16} & \multirow{2}{*}{ Suzaku } & On & 508078010 & 2013-08-07 & 170.58 & -37.28 & 82.3 & 49.5 & 24.1 \\
\hline & & Off & 508073010 & 2013-08-09 & 165.84 & -38.39 & 83.1 & 50.6 & 6.3 \\
\hline \multirow[t]{2}{*}{ MBM 20} & \multirow[t]{2}{*}{ XMM-Newton } & On & 0203900201 & $2004-08-23$ & 211.37 & -36.57 & 101.1 & $54.1,59.7,29.3$ & 17.6 \\
\hline & & Off & 0203900101 & 2004-08-09 & 213.40 & -39.11 & 109.4 & $72.8,74.3,62.8$ & 2.3 \\
\hline \multirow[t]{2}{*}{ MBM 20} & \multirow[t]{2}{*}{ Suzaku } & On & 502075010 & $2008-02-11$ & 211.41 & -36.56 & 107.1 & 71.8 & 19.1 \\
\hline & & Off & 502076010 & $2007-07-30$ & 213.42 & -39.10 & 103.8 & 83.6 & 2.2 \\
\hline \multirow[t]{2}{*}{$\mathrm{G} 236+38$} & \multirow[t]{2}{*}{ Suzaku } & On & 506055010 & 2011-06-01 & 235.95 & $\begin{array}{r}38.21 \\
+38.21\end{array}$ & 69.8 & 41.8 & 12.2 \\
\hline & & Off & 506056010 & 2011-06-07 & 237.09 & +41.11 & 70.8 & 41.6 & 1.4 \\
\hline \multirow[t]{2}{*}{ Filament } & \multirow[t]{2}{*}{ XMM-Newton } & On & 0084960201 & 2002-05-03 & 278.67 & -45.32 & 10.0 & $11.8,11.8,7.9$ & 7.6 \\
\hline & & Off & 0084960101 & 2002-05-03 & 278.73 & -47.09 & 25.1 & $4.5,4.1,0.9$ & 2.0 \\
\hline \multirow[t]{2}{*}{ Filament } & \multirow[t]{2}{*}{ Suzaku } & On & 501002010 & 2006-03-03 & 278.64 & -45.30 & 101.5 & 72.4 & 8.3 \\
\hline & & Off & 501001010 & 2006-03-01 & 278.69 & -47.07 & 80.1 & 60.9 & 2.0 \\
\hline
\end{tabular}

Note. - The shadows are tabulated in order of increasing Galactic longitude. Columns 6 and 7 contain the observation pointing direction in Galactic coordinates. The nominal exposure (column 8) is the exposure time taken from the header of the unfiltered events list. The usable exposure (column 9) is the exposure time taken from the header of the spectrum file, extracted after the data have been filtered (Sections 2.1 and 2.2). For the XMM-Newton observations, the listed values are the usable MOS1, MOS2, and pn exposures, respectively. The absorbing column density, $N_{\mathrm{H}}$ (column 10), was calculated from the average 100-micron intensity within the field of view (see Section 3 ).
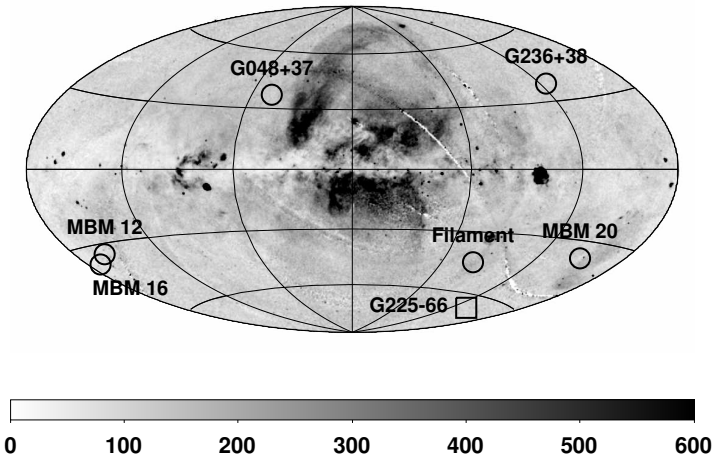

Figure 1. ROSAT All-Sky Survey map of the $3 / 4 \mathrm{keV}$ SXRB (units: $10^{-6}$ counts s$^{-1}$ arcmin $^{-2}$; [Snowden et al. [1997), centered on the Galactic Center, showing the locations of the shadows analyzed in this paper (circles), and of shadow G225-66 (square; Henley et al. 2015a).

Newton and Suzaku observations, respectively.

\subsection{XMM-Newton Data Reduction}

The XMM-Newton data reduction and spectral extraction, which we carried out with the XMM-Newton Extended Source Analysis Software ${ }^{2}$ (XMM-ESAS; Snowden \& Kuntz 2013) as distributed with version 13.5.0 of the Science Analysis System ${ }^{3}$ (SAS), was generally similar to that described in Henley \& Shelton (2012). However, note that here we used data from the pn camera (Strüder et al. 2001), in addition to data from the MOS cameras (Turner et al. 2001). For each observation, we first used the standard SAS emchain and epchain scripts to produce calibrated events lists from each camera, and then used the $X M M$-ESAS mos-filter and pn-filter

\footnotetext{
2 http://heasarc.gsfc.nasa.gov/docs/xmm/xmmhp_xmmesas.html
}

3 http://xmm.esac.esa.int/sas/ scripts to remove from the data periods of elevated count rate, due to soft-proton flaring. The amounts of good time remaining after this filtering are shown in column 9 of Table 1. Note that the resulting pn exposures are shorter than the corresponding MOS exposures. As discussed in Henley et al. (2014), this is likely due to the greater sensitivity of the pn detector, meaning that relatively smaller departures from the mean count rate are flagged as soft proton flares.

For most observations we used data from the Second $X M M-N e w t o n$ Serendipitous Source Catalog $\left(2 \mathrm{XMM}^{4}\right.$; Watson et al. 2009) to identify point sources with 0.5 $2.0 \mathrm{keV}$ fluxes exceeding $1 \times 10^{-14} \mathrm{erg} \mathrm{cm}^{-2} \mathrm{~s}^{-1}$. This is a lower source removal threshold than that used in Henley \& Shelton (2012), but it is the threshold used in our XMM-Newton survey of the halo emission (HS13). However, the observations of G048+37 are not included in the 2XMM catalog, and so for these observations we ran the source detection ourselves, using the SAS edetect_chain script. In all cases, the detected sources were excised from the data using circles of radius $50^{\prime \prime}$. A visual inspection indicated that there were no bright or extended sources in the fields that would have needed larger source removal regions.

We identified and excluded MOS CCDs that exhibited the anomalous state identified by Kuntz \& Snowden (2008). Also, we found that, for the on-MBM 20 observation (0203900201), the exposure time for the first (upper left, in detector coordinates) quadrant of the pn detector is $\sim 40 \%$ of those for the other three quadrants. It is unclear why this is the case. We decided to exclude this quadrant of the pn detector from the subsequent processing and analysis of this observation.

Having excluded point sources and anomalous CCDs, we used the $X M M$-ESAS mos-spectra and pn-spectra

${ }^{4}$ Specifically, we used data from the 2XMMi DR3 data release; http://xmmssc-www.star.le.ac.uk/Catalogue/2XMMi-DR3/ 


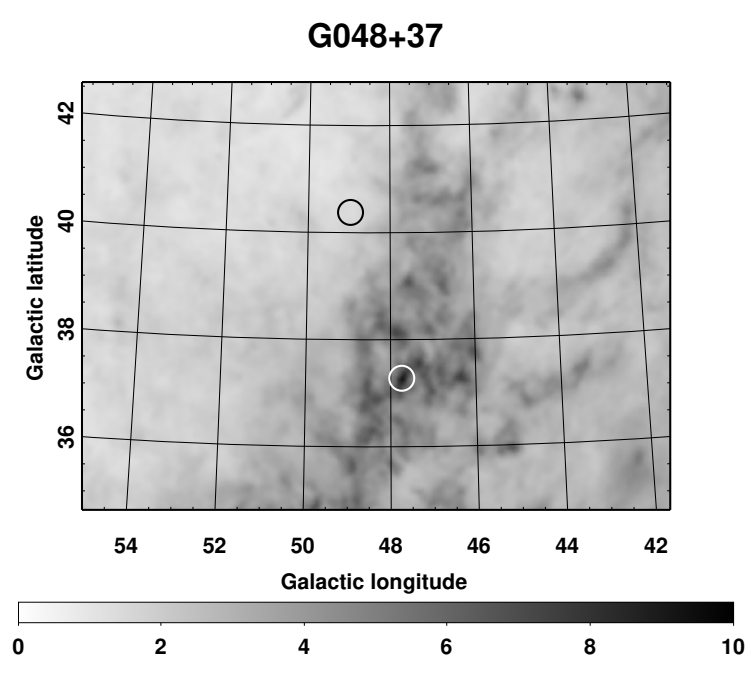

MBM 16

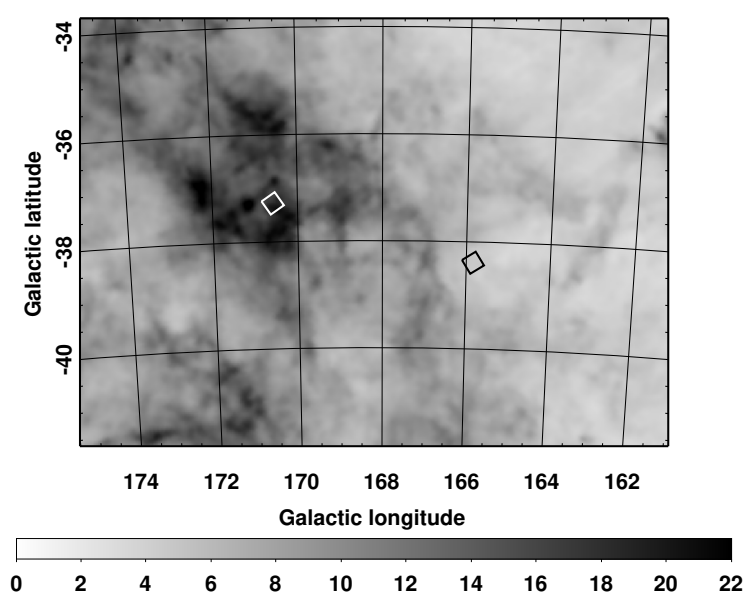

G236+38

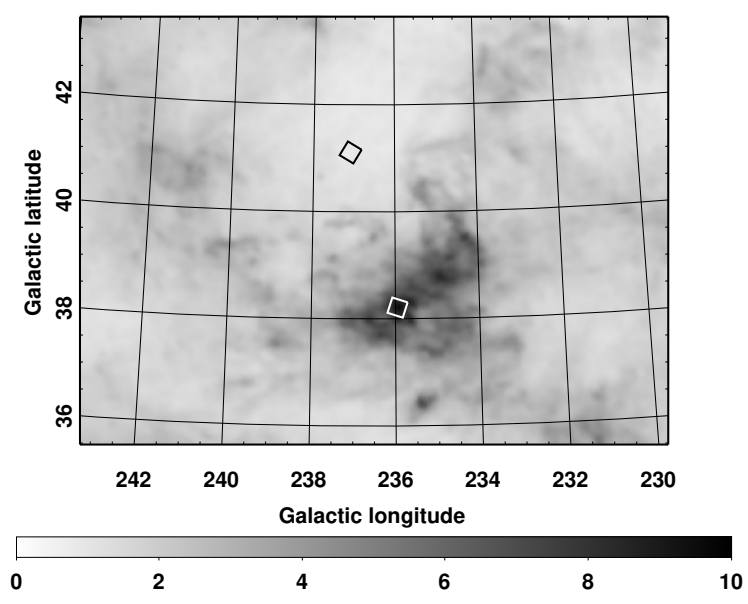

MBM 12

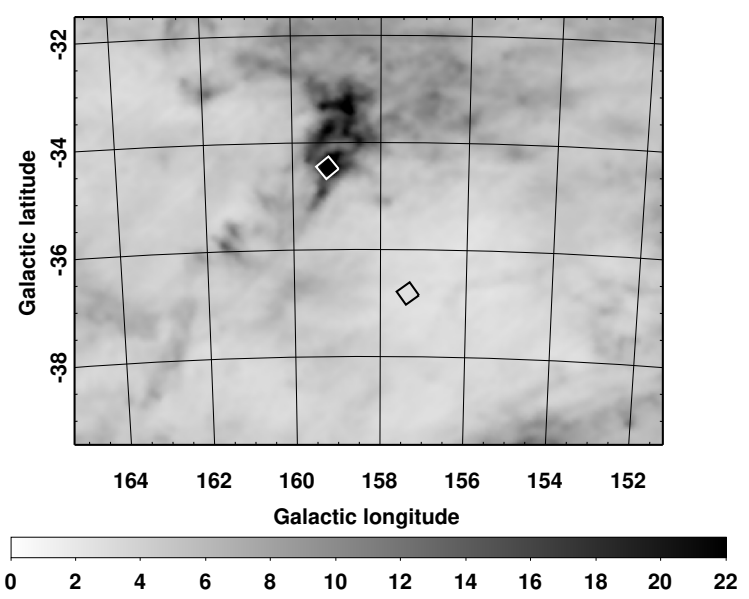

MBM 20

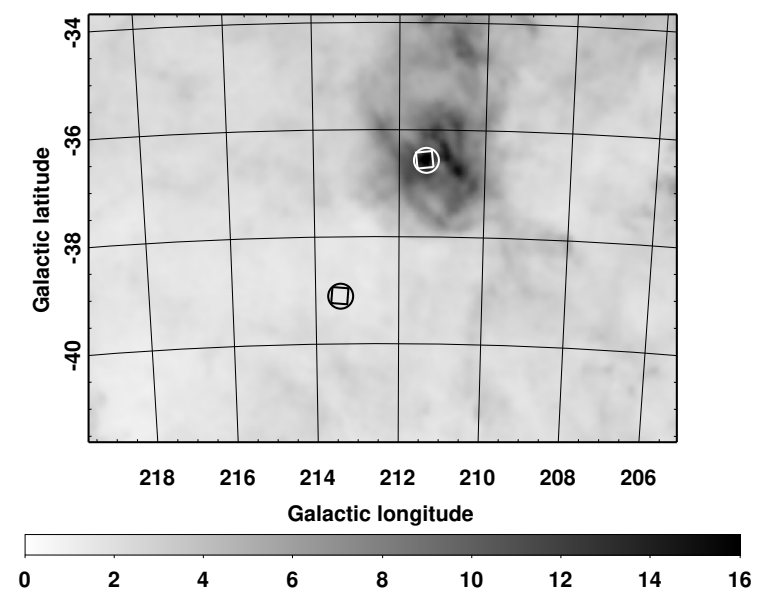

Filament

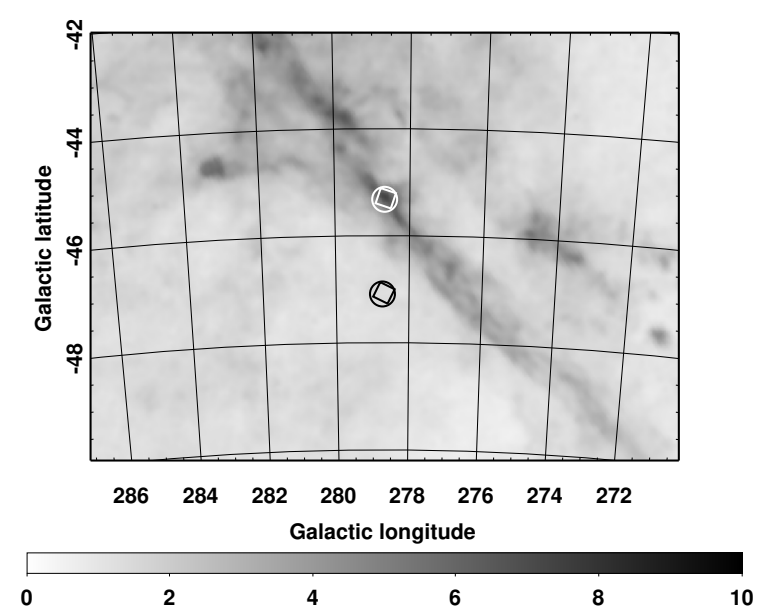

Figure 2. DIRBE-corrected IRAS 100-micron maps of the shadowing clouds studied here (Schlegel et al. 1998). The grayscales are in $\mathrm{MJy} \mathrm{sr}^{-1}$. The circles (radius $=14^{\prime}$ ) and squares $\left(18^{\prime} \times 18^{\prime}\right)$ indicate the approximate XMM-Newton and Suzaku fields of view, respectively. 
scripts to extract SXRB spectra from the full remaining fields of view of the MOS and pn cameras, respectively, and used the mos_back and pn_back programs to calculate corresponding quiescent particle background (QPB) spectra. The QPB spectra were calculated from a database of filter-wheel-closed data, scaled to our observations using data from the unexposed regions of the cameras (Kuntz \& Snowden 2008). The spectral extraction scripts also calculated the response files - the redistribution matrix file (RMF) and ancillary response file (ARF) - needed for each spectrum, using the SAS rmfgen and arfgen programs, respectively. Before carrying out our spectral analysis, we grouped each SXRB spectrum such that there were at least 50 counts per bin, and then subtracted the corresponding QPB spectrum.

\subsection{Suzaku Data Reduction}

For the Suzaku observations, we used only the data from the back-illuminated (BI) XIS1 camera, which is more sensitive to soft X-rays than the front-illuminated (FI) XIS cameras (Koyama et al. 2007). We processed the Suzaku data using HEASoft ${ }^{5}$ version 6.15.1. For each observation, we first ran the aepipeline script to produce a calibrated and screened XIS1 events list. We then combined the data taken in the $3 \times 3$ and $5 \times 5$ modes, and applied further screening to the data, in addition to the standard screening described in the Suzaku ABC Guide. ${ }^{6}$ In particular, in order to reduce the non-X-ray background (NXB), we kept only times when the geomagnetic cut-off rigidity (COR) exceeded $8 \mathrm{GV}$, and in order to reduce contamination from solar X-rays scattered off the Earth's atmosphere, we kept only times when the angle between Suzaku's sight line and the limb of the Earth, ELV, exceeded $10^{\circ}$ (cf. the standard screening criterion is ELV $>5^{\circ}$ ). For most observations, we applied the standard screening for the angle between Suzaku's sight line and the limb of the sunlit Earth, DYE_ELV, and kept only times when DYE_ELV $>20^{\circ}$. However, Sekiya et al. (2014) showed that, with this DYE_ELV threshold, $\mathrm{O}$ I emission at $E=0.525 \mathrm{keV}$ from the fluorescence of atmospheric oxygen can significantly contaminate the astrophysical O VII $\mathrm{K} \alpha$ emission at $E \approx 0.57 \mathrm{keV}$ in Suzaku spectra taken after 2011 . This contamination can be removed by increasing the DYE_ELV threshold from $20^{\circ}$ to $60^{\circ}$. As our G236+38 observations were taken in mid-2011, near the time of the increased atmospheric O I contamination reported by Sekiva et al. (2014), we decided to err on the side of caution and applied this stricter DYE_ELV screening criterion to the G236+38 observations. We also applied this stricter screening to the MBM 16 observations, which were taken in 2013. For both sets of observations, this stricter screening reduced the usable exposure time by about one quarter. The usable exposures remaining after the screening of the Suzaku data are shown in column 9 of Table 1 .

We have previously found that automated source detection software does not work well on Suzaku images, presumably because of Suzaku's broad point-spread function (Henley \& Shelton 2008). We therefore excluded sources from the Suzaku data by hand. For the

\footnotetext{
5 http://heasarc.gsfc.nasa.gov/lheasoft/

6 http://heasarc.gsfc.nasa.gov/docs/suzaku/analysis/abc/abc.html
}

MBM 12 observations, we used the same source exclusion regions described in Smith et al. (2007). For the filament and MBM 20 observations, we used a similar procedure to that described in Henley \& Shelton (2008), though here we used more stringent source exclusion thresholds. We used data from the 2XMM source catalog (Watson et al. 2009) ${ }^{7}$ to identify sources in the Suzaku fields with $0.5-2.0 \mathrm{keV}$ fluxes exceeding $2 \times 10^{-14} \mathrm{erg} \mathrm{cm} \mathrm{cm}^{-2} \mathrm{~s}^{-1}$. Sources with fluxes of $(2-10) \times 10^{-14}$ and $>1 \times 10^{-13} \mathrm{erg} \mathrm{cm}^{-2} \mathrm{~s}^{-1}$ were excised from the data using circles of radius 1.5 and 2.5 , respectively. The MBM 16 and G236+38 observations are not covered by the 2XMM catalog. We identified by eye one bright source each in the on- and off-MBM 16 observations, at $(\alpha, \delta)=\left(03^{\mathrm{h}} 19^{\mathrm{m}} 09^{\mathrm{s}},+11^{\circ} 43^{\prime} 15^{\prime \prime}\right)$ and $\left(03^{\mathrm{h}} 04^{\mathrm{m}} 12^{\mathrm{s}},+13^{\circ} 07^{\prime} 42^{\prime \prime}\right)$, respectively. We excluded these sources with $2^{\prime}$ circles. We identified by eye two bright sources in the on-G236+38 observation, at $(\alpha, \delta)=\left(09^{\mathrm{h}} 46^{\mathrm{m}} 02^{\mathrm{s}},+00^{\circ} 35^{\prime} 25^{\prime \prime}\right)$ and $\left(09^{\mathrm{h}} 46^{\mathrm{m}} 34^{\mathrm{s}},+00^{\circ} 28^{\prime} 07^{\prime \prime}\right)$. We excluded these sources with $4^{\prime}$ circles (for both MBM 16 and G236+38, the source exclusion radii were chosen by eye). No bright point sources were apparent in the off-G236+38 observation.

Although we identified the sources to be excluded from our Suzaku observations using sky (J2000) coordinates, we carried out the subsequent spectral extraction, NXB calculation, and ARF calculation (see below) using XIS detector coordinates. Therefore, we converted the sources' coordinates to detector coordinates using aecoordcalc. The conversion from sky to detector coordinates varies as a function of time, depending on the spacecraft's attitude. We carried out the conversion using the mid-time of each observation. For a few sources, the resulting source exclusion region in detector coordinates was not perfectly aligned with the image of the source. In such cases, we adjusted the location of the exclusion region by hand - these adjustments were typically $\lesssim 20^{\prime \prime}$.

We extracted SXRB spectra from the full field of view of the XIS1 camera, minus the excluded sources described above. We did not exclude the regions of the detector illuminated by the Fe-55 calibration sources, as the manganese $\mathrm{K}$ lines produced by these sources lie above the energy band we are interested in. ${ }^{8}$ As with the XMM-Newton spectra, we grouped each Suzaku SXRB spectrum such that were at least 50 counts per bin.

From each SXRB spectrum we subtracted a corresponding NXB spectrum, calculated using xisnxbgen (Tawa et al. 2008). The NXB spectra were constructed from night-Earth observations with the same COR distributions as the SXRB observations. We also calculated the RMF and ARF needed for each spectrum, using xisrmfgen and xissimarfgen (Ishisaki et al. 2007), respectively. The latter tool takes into account the contamination on the XIS1 detector's optical blocking filter, which reduces the detector's low-energy sensitivity

7 See footnote 4

8 Manganese also produces L-shell lines at $\sim 640 \mathrm{eV}$ (Kortright \& Thompson 2009), within our energy band of interest. However, while the Suzaku NXB spectra clearly exhibit the manganese $\mathrm{K}$ lines, the $\mathrm{L}$ lines are not apparent, implying that contamination from these lines is not significant. 
(Koyama et al. 2007). For the ARF calculation, we assumed a uniform source of radius $20^{\prime}$.

\section{SPECTRAL MODEL DESCRIPTION}

In order to separate the foreground and halo emission, we used XSPEC version 12.8.11 (Arnaud 1996) to fit an SXRB spectral model to the on- and off-shadow spectra from each pair of observations in Table 1 . Our spectral model is essentially the same as that used by Henley et al. (2015a), and consists of components representing (1) the foreground emission, (2) the Galactic halo emission, (3) the extragalactic background emission, and (4) (for XMM-Newton spectra only) components of the particle background not removed by the filtering and QPB subtraction described in Section 2.1. In most regards, the on- and off-shadow models for each pair of observations were the same, apart from the absorbing column used to attenuate the halo and extragalactic components - this difference between the on- and offshadow models is the key to separating the foreground and halo emission.

We briefly describe the model components below, referring the reader to Henley et al. (2015a) for more details. We also describe a modification to our basic spectral model that was necessary for the on-MBM 12 Suzaku spectrum.

(1) We examined three different foreground models, representing limits in which SWCX emission or LB emission dominate the foreground. For our reference spectral model, we used the C14-SWCX model from Henley et al. (2015a), which is based on CX line ratio data from Cumbee et al. (2014). This model consisted of $\mathrm{K} \alpha-\delta$ lines from $\mathrm{C} \mathrm{V}^{9}$ and $\mathrm{K} \alpha-\epsilon$ lines from $\mathrm{C}$ VI, O VII, and $\mathrm{O}$ VIII. For each ion, the lines' intensities were tied to those of a reference line using the Cumbee et al. (2014) line intensity ratios. The overall normalization for each ion's line emission was a free parameter. Note that, because of the relatively poor spectral resolution of the XMM-Newton and Suzaku CCD spectrometers at low energies, we did not include nitrogen lines in this foreground model (the N VI and N VII K $\alpha$ lines lie between those of C VI and O VII).

We also investigated a second SWCX foreground model, based on the AtomDB Charge Exchange code (ACX; Smith et al. 2014), which uses analytical expressions to calculate the distributions of principal quantum number, $n$, and orbital angular momentum, $l$, for the electron that transfers from the donor atom to the receiving ion (the user has a number of such expressions to choose from). Following Henley et al. (2015a), we refer to this model as the ACX-SWCX foreground model. The relative strengths of lines from different ions are controlled via an ionization temperature parameter, assuming collisional ionization equilibrium (CIE). This temperature was a free parameter in the fitting. We used the same settings for the ACX model component as Henley et al. (2015a), i.e., swcx $=1$ (suitable for studying SWCX emission) and model $=8$ (meaning that captured electrons are distributed between the two nearest $n$ shells to the most-probable shell, and that the Sep-

\footnotetext{
${ }^{9}$ Henley et al. (2015a) did not include these lines in their version of the model-they cut off their spectra at $0.4 \mathrm{keV}$, whereas these lines are at $0.299-0.379 \mathrm{keV}$.
}

arable distribution is used for the $l$ levels; Smith et al. 2014; Smith \& Foster 2014). These are also the settings favored by Smith et al. (2014) for modeling SWCX emission. However, it should be noted that the expressions used by the ACX model cannot be expected to accurately model the $n$ and $l$ distributions for all relevant $\mathrm{CX}$ reactions. We will discuss the reliability of the ACX model in Section 5.1

The third and final foreground model assumed that the emission was from hot plasma in the LB, rather than from SWCX. While such a model may not be realistic, as SWCX emission likely dominates the foreground emission in the XMM-Newton/Suzaku band (Koutroumpa et al. 2007, 2009, 2011), it does allow our results to be compared with previous shadowing studies that have used such a model Galeazzi et al. 2007; Lei et al. 2009; Gupta et al. 2009). For this LB model, we modeled the foreground emission with a singletemperature (1T) APEC thermal plasma model, assuming CIE (Smith et al. 2001; Foster et al. 2012). The temperature and normalization of this model were free parameters.

Note that our spectral modeling assumes that the foreground emission is identical in the on- and off-shadow spectra. For most of our shadowing clouds, the on- and off-shadow pointings took place within a few days of each other (Table 1). Assuming that the foreground emission is approximately constant over such a time scale is not unreasonable (although it should be noted that variations in the SWCX emission have been observed over shorter times; Snowden et al.|2004; Fujimoto et al.|2007; Carter \& Sembav 2008; Carter et al. 2010; Ezoe et al. 2010; Wargelin et al. 2014). For the XMM-Newton and Suzaku observations of MBM 20, the two pointings were two weeks and $\sim$ six months apart, respectively (Table1). Over such periods of time, the solar wind conditions and/or the viewing geometry through the heliosphere could change significantly, resulting in large changes in the SWCX emission, which would adversely affect the spectral analysis. We will discuss this in Section 5.4.2.

(2) We modeled the halo emission using a $1 T$ APEC model, assuming CIE. In general, the temperature and normalization of this component were free parameters. However, Ursino et al. (2014) reported the non-detection of halo emission in the direction of MBM 16, with an upper limit on the emission measure of $\sim 10^{-3} \mathrm{~cm}^{-6} \mathrm{pc}$. In our survey of the halo emission with $X M M-N e w t o n$ (HS13), we did not detect halo emission on $\sim 1 / 5$ of our sight lines; for these sight lines, the lower and upper quartiles of the emission measure upper limits were $0.8 \times 10^{-3}$ and $1.4 \times 10^{-3} \mathrm{~cm}^{-6} \mathrm{pc}$, respectively. Here, we found that, if the halo temperature was a free parameter in the MBM 16 fits, we typically could not constrain this parameter. Therefore, for MBM 16 only, we fixed the halo temperature at $2.1 \times 10^{6} \mathrm{~K}$ (a typical value; HS13).

The halo component was subject to absorption, using the XSPEC phabs model (Bałucińska-Church \& McCammon 1992; Yan et al. 1998). For each observation, we calculated the absorbing column density, $N_{\mathrm{H}}$, by first calculating the average value of $I_{100}$ in the field view (Schlegel et al. 1998), and then converting this value to $N_{\mathrm{H}}$ using the conversion relations from Snowden et al. (2000). The resulting column densities are shown in column 10 of Table 1 . 
The advantage of the $I_{100}$ data over $N(\mathrm{H} \mathrm{I})$ data (e.g., Kalberla et al. 2005) is that the former have higher spatial resolution, allowing us to account for variations in the amount of absorbing material over the X-ray fields of view. Using the HEASARC $N_{\mathrm{H}}$ tool $^{10}$ results in on-cloud column densities that are typically $\sim 2-4$ times smaller than those tabulated in Table 1, due to its averaging over an area larger than that covered by the X-ray detectors. Note that the column densities for the filament observations are different from those used in Henlev et al. (2007), Henlev \& Shelton (2008), and Lei et al. (2009), as in those studies we did not average $I_{100}$ over the fields of view.

(3) We modeled the extragalactic emission using a double broken power-law (Smith et al. 2007). For the XMMNewton spectra, the presence of soft proton contamination (see (4), below) meant that we could not independently constrain the normalization of this model, and so it was necessary to fix this normalization. We rescaled Smith et al.'s (2007) nominal extragalactic model so that its $0.5-2.0 \mathrm{keV}$ surface brightness matched that expected from sources below the source removal flux threshold (HS13). For the Suzaku spectra, which do not suffer from soft proton contamination, we allowed the overall normalization of this model to be a free parameter. For a given shadow, the normalizations for the on- and offshadow directions were independent. The extragalactic model was attenuated by the same absorbing columns as the halo component (see above).

(4) For the XMM-Newton spectra only, we added Gaussians at $\sim 1.49$ (pn and MOS spectra) and $\sim 1.75 \mathrm{keV}$ (MOS spectra only) representing the $\mathrm{Al}$ and $\mathrm{Si}$ instrumental lines, which were not removed by the QPB subtraction (Kuntz \& Snowden 2008). As the filtering described in Section 2.1 does not remove all the soft proton contamination from the XMM-Newton spectra, we also added a power-law that was not folded through the instrumental response to model any residual contamination (Kuntz \& Snowden 2008; Snowden \& Kuntz 2013). The parameters of these additional model components were independent for each individual exposure that was analyzed.

The on-MBM 12 Suzaku field includes the intermediate polar XY Ari, which we excised from the data using a circle of radius $2^{\prime}$ (following Smith et al. 2007). However, Smith et al. (2007) found that this source is so bright that X-rays from the wings of the point spread function significantly contaminate the SXRB spectrum above $\sim 1 \mathrm{keV}$. Like Smith et al., we modeled this contamination by adding an absorbed bremmstrahlung component to the on-MBM 12 spectral model. This component typically had a best-fit temperature of $k T=200 \mathrm{keV}$ (this is the default upper limit of the temperature in the XSPEC bremss model), and a hydrogen column density of $\sim(5-7) \times 10^{22} \mathrm{~cm}^{-2}$ (similar to the values found by Smith et al. 2007). As the XY Ari contamination component contributes very little emission below $1 \mathrm{keV}$, the details of this model should not significantly affect our measurements of the foreground and halo SXRB emission.

Many previous XMM-Newton or Suzaku studies of the SXRB emission have assumed abundances (e.g.,

10 http://heasarc.gsfc.nasa.gov/cgi-bin/Tools/w3nh/w3nh.pl
Anders \& Grevesse 1989, hereafter AG89) in which the oxygen abundance relative to hydrogen is $\sim 8 \times 10^{-4}$ (Smith et al. 2007; Galeazzi et al. 2007; Gupta et al. 2009; Yosh09; Henlev et al. 2010, 2015a; HS13). This choice was often in part to allow easier comparisons with preceding studies. However, recent measurements of the solar oxygen abundance yield a value about 0.2 dex lower: $4.90 \times 10^{-4}$ (Asplund et al. 2009, hereafter Aspl09). Since oxygen emission dominates the halo emission in the XMM-Newton/Suzaku band, the assumed oxygen abundance will affect the halo emission measures inferred from the spectral analysis. Here, we decided to use the Aspl09 solar abundances as our reference abundances. However, to allow our results to be compared with earlier studies, we also carried out fits assuming AG89 solar abundances. Note that, because of its higher carbon, nitrogen, and oxygen abundances, using the AG89 abundance table not only results in more soft X-ray emission per unit emission measure from the hot gas, but also in greater photoelectric absorption cross-sections in the cold gas. In addition, to further test the sensitivity of our results to the assumed abundances, we carried out fits using the Wilms et al. (2000, hereafter Wilm00) interstellar abundances. (Note that the Wilm00 oxygen abundance is the same as the Aspl09 value. However, the carbon and nitrogen abundances, which are also relevant to emission in the XMM-Newton / Suzaku band, are 0.05 dex lower and 0.05 dex higher in Wilm00 than in Aspl09, respectively.)

For each pair of XMM-Newton observations, we fitted the above-described model simultaneously to the 0.3 $5.0 \mathrm{keV}$ MOS and $0.4-5.0 \mathrm{keV}$ pn spectra from the on and off-shadow directions (the pn data are generally not usable below $0.4 \mathrm{keV}$; Snowden \& Kuntz 2013). In general, for each pair of Suzaku observations, we fitted the model simultaneously to the on- and off-shadow $0.3-5.0 \mathrm{keV}$ XIS1 spectra. The exceptions are the G236+38 and MBM 16 observations. In the G236+38 XIS1 spectra, we found that the count rates increased unexpectedly below $\sim 0.35 \mathrm{keV}$, resulting in poor fits. This increase is unexpected because the XIS1 camera's sensitivity is small below $\sim 0.35 \mathrm{keV}$-because of the build up of contamination on the camera's optical blocking filter, the XIS1 low-energy sensitivity is smaller for the G236+38 observations than for observations taken earlier in the mission. It is possible that the NXB is inaccurately modeled in the G236+38 spectra at low energies. Therefore, for G236+38, we cut off the XIS1 spectra at $0.35 \mathrm{keV}$, rather than $0.3 \mathrm{keV}$. Similarly, we found that the MBM 16 XIS1 count rates increased unexpectedly below $\sim 0.4 \mathrm{keV}$, and so for these spectra we placed the low-energy cut-off at $0.4 \mathrm{keV}$. Because of this higher cut-off, we did not include the C V lines in the C14-SWCX model when fitting to the MBM 16 spectra.

\section{SPECTRAL ANALYSIS RESULTS}

Our spectral fit results are presented in Table 2 The headings within the body of the table indicate the foreground model and abundances used for each set of results. Note in particular that the results from our reference model are in Section (a) of the table. For the C14-SWCX model, the foreground $\mathrm{K} \alpha$ intensities for $\mathrm{C} \mathrm{V}$ (if included), C VI, O VII, and O VIII are shown in Columns 4-7, respectively, in line units (L.U. = photons $\left.\mathrm{cm}^{-2} \mathrm{~s}^{-1} \mathrm{sr}^{-1}\right)$. For the ACX-SWCX and LB 
Table 3

Foreground and Halo Surface Brightnesses

\begin{tabular}{|c|c|c|c|c|c|c|c|c|c|c|c|c|}
\hline \multirow[b]{2}{*}{$\begin{array}{c}\text { Shadow }{ }^{\mathrm{a}} \\
\text { (1) }\end{array}$} & \multicolumn{4}{|c|}{ C14-SWCX model } & \multicolumn{4}{|c|}{ ACX-SWCX model } & \multicolumn{4}{|c|}{ LB model } \\
\hline & \multicolumn{2}{|r|}{$\begin{array}{l}S_{\mathrm{fg}}^{0.4-1.0} \\
(2)\end{array}$} & \multicolumn{2}{|c|}{$\begin{array}{c}S_{\mathrm{h}}^{0.5-2.0} \\
(3)\end{array}$} & \multicolumn{2}{|c|}{$\begin{array}{l}S_{\mathrm{fg}}^{0.4-1.0} \\
\quad(4)\end{array}$} & \multicolumn{2}{|c|}{$\begin{array}{c}S_{\mathrm{h}}^{0.5-2.0} \\
(5)\end{array}$} & \multicolumn{2}{|c|}{$\begin{array}{l}S_{\mathrm{fg}}^{\mathrm{o.4}}(6)-1.0 \\
(6)\end{array}$} & \multicolumn{2}{|c|}{$\begin{array}{c}S_{\mathrm{h}}^{0.5-2.0} \\
(7)\end{array}$} \\
\hline \multicolumn{13}{|c|}{ Asplund et al. (2009) abundances } \\
\hline $\mathrm{G} 048+37(\mathrm{X})$ & 1.25 & $(1.14,1.65)$ & 6.28 & $(5.83,6.84)$ & 2.18 & $(1.89,2.82)$ & 5.03 & $(4.64,5.58)$ & 2.08 & $(1.75,2.54)$ & 5.50 & $(4.90,5.78)$ \\
\hline MBM $12(\mathrm{~S})$ & 1.09 & $(0.90,1.25)$ & 1.04 & $(0.85,1.65)$ & 1.26 & $(1.07,1.43)$ & 1.26 & $(0.96,1.83)$ & 1.31 & $(1.13,1.48)$ & 0.94 & $(0.53,1.45)$ \\
\hline MBM $16(\mathrm{~S})$ & 3.11 & $(2.60,3.51)$ & 0.04 & $(0.00,0.93)$ & 3.29 & $(2.89,3.58)$ & 0.00 & $(0.00,0.62)$ & 2.66 & $(2.27,3.05)$ & 1.15 & $(0.48,1.81)$ \\
\hline MBM $20(\mathrm{X})$ & 0.10 & $(0.09,0.13)$ & 2.98 & $(2.89,3.02)$ & 0.21 & $(0.18,0.25)$ & 3.02 & $(2.94,3.19)$ & 0.16 & $(0.14,0.20)$ & 2.88 & $(2.82,2.94)$ \\
\hline MBM $20(\mathrm{~S})$ & 0.52 & $(0.11,0.78)$ & 2.19 & $(1.69,3.49)$ & 0.34 & $(0.19,0.49)$ & 2.51 & $(2.05,3.00)$ & 0.64 & $(0.45,0.83)$ & 2.25 & $(1.90,2.59)$ \\
\hline $\mathrm{G} 236+38(\mathrm{~S})$ & 2.21 & $(1.05,3.38)$ & 4.63 & $(2.84,6.54)$ & 1.44 & $(1.02,1.86)$ & 5.70 & $(4.84,6.56)$ & 3.79 & $(2.24,4.91)$ & 3.43 & $(1.96,6.61)$ \\
\hline Filament (X) & 1.79 & $(1.30,2.14)$ & 7.37 & $(6.57,7.66)$ & 1.72 & $(1.19,2.45)$ & 7.60 & $(6.78,8.64)$ & 3.78 & $(2.90,4.31)$ & 5.21 & $(4.80,6.93)$ \\
\hline Filament (S) & 0.13 & $(0.04,0.48)$ & 5.80 & $(5.15,6.03)$ & 0.22 & $(0.09,0.35)$ & 5.76 & $(5.48,6.04)$ & 0.39 & $(0.19,0.93)$ & 5.56 & $(4.72,5.99)$ \\
\hline \multicolumn{13}{|c|}{ Anders \& Grevesse (1989) abundances } \\
\hline $\mathrm{G} 048+37(\mathrm{X})$ & 2.28 & $(2.05,2.59)$ & 5.78 & $(5.29,6.34)$ & 3.48 & $(3.10,3.76)$ & 4.17 & $(3.88,4.68)$ & 3.30 & $(2.97,3.68)$ & 4.80 & $(4.42,5.63)$ \\
\hline MBM $12(\mathrm{~S})$ & 1.17 & $(1.02,1.32)$ & 1.26 & $(0.78,1.86)$ & 1.47 & $(1.30,1.64)$ & 1.27 & $(0.94,2.11)$ & 1.40 & $(1.24,1.55)$ & 1.17 & $(0.65,1.74)$ \\
\hline MBM $16(\mathrm{~S})$ & 3.28 & $(2.84,3.62)$ & 0.25 & $(0.00,1.16)$ & 3.53 & $(3.19,3.82)$ & 0.00 & $(0.00,0.60)$ & 2.74 & $(2.38,3.10)$ & 1.75 & $(1.03,2.52)$ \\
\hline MBM $20(\mathrm{X})$ & 0.49 & $(0.45,0.53)$ & 2.98 & $(2.94,3.09)$ & 0.28 & $(0.24,0.33)$ & 3.24 & $(3.13,3.28)$ & 0.62 & $(0.58,0.67)$ & 2.85 & $(2.79,2.93)$ \\
\hline MBM $20(\mathrm{~S})$ & 0.86 & $(0.60,1.10)$ & 2.22 & $(1.94,2.49)$ & 0.49 & $(0.34,0.65)$ & 2.88 & $(2.58,3.18)$ & 0.92 & $(0.72,1.28)$ & 2.43 & $(2.02,2.85)$ \\
\hline $\mathrm{G} 236+38(\mathrm{~S})$ & 3.41 & $(2.43,4.48)$ & 3.96 & $(2.31,6.53)$ & 1.83 & $(1.40,2.27)$ & 6.12 & $(5.11,7.13)$ & 4.67 & $(3.58,5.63)$ & 3.13 & $(2.38,5.14)$ \\
\hline Filament (X) & 1.70 & $(1.35,1.90)$ & 8.76 & $(8.23,9.51)$ & 3.16 & $(2.48,3.70)$ & 6.77 & $(6.03,7.70)$ & 3.82 & $(3.48,4.17)$ & 6.18 & $(5.08,6.98)$ \\
\hline Filament (S) & 0.58 & $(0.18,0.99)$ & 6.03 & $(5.73,6.26)$ & 0.38 & $(0.25,0.51)$ & 6.41 & $(6.09,6.72)$ & 1.16 & $(0.64,1.67)$ & 5.46 & $(4.52,6.46)$ \\
\hline \multicolumn{13}{|c|}{ Wilms et al. (2000) abundances } \\
\hline $\mathrm{G} 048+37(\mathrm{X})$ & 1.48 & $(1.36,1.73)$ & 6.14 & $(5.47,6.50)$ & 2.48 & $(2.17,2.89)$ & 4.75 & $(4.36,5.20)$ & 2.39 & $(2.01,2.77)$ & 5.28 & $(4.89,5.95)$ \\
\hline MBM $12(\mathrm{~S})$ & 1.08 & $(0.92,1.23)$ & 1.10 & $(0.73,1.66)$ & 1.30 & $(1.12,1.49)$ & 1.24 & $(0.93,1.92)$ & 1.34 & $(1.16,1.51)$ & 0.97 & $(0.57,1.50)$ \\
\hline MBM 16 (S) & 3.15 & $(2.65,3.43)$ & 0.02 & $(0.00,0.90)$ & 3.33 & $(2.94,3.61)$ & 0.00 & $(0.00,0.60)$ & 2.73 & $(2.33,3.12)$ & 1.14 & $(0.47,1.80)$ \\
\hline MBM $20(\mathrm{X})$ & 0.11 & $(0.10,0.14)$ & 3.04 & $(2.95,3.11)$ & 0.18 & $(0.15,0.21)$ & 2.92 & $(2.87,2.99)$ & 0.26 & $(0.23,0.30)$ & 2.94 & $(2.84,2.98)$ \\
\hline MBM $20(\mathrm{~S})$ & 0.55 & $(0.25,0.83)$ & 2.18 & $(1.68,3.51)$ & 0.37 & $(0.22,0.53)$ & 2.52 & $(2.06,3.01)$ & 0.71 & $(0.52,0.91)$ & 2.24 & $(1.88,2.59)$ \\
\hline $\mathrm{G} 236+38(\mathrm{~S})$ & 2.30 & $(1.47,3.45)$ & 4.59 & $(2.79,6.52)$ & 1.54 & $(1.11,1.97)$ & 5.70 & $(4.82,6.58)$ & 3.74 & $(2.49,5.07)$ & 3.67 & $(1.90,6.32)$ \\
\hline Filament (X) & 1.33 & $(1.07,1.67)$ & 8.06 & $(7.21,8.66)$ & 2.41 & $(1.83,2.97)$ & 6.79 & $(6.01,7.32)$ & 3.76 & $(3.39,4.39)$ & 5.53 & $(4.58,5.98)$ \\
\hline Filament (S) & 0.15 & $(0.10,0.29)$ & 5.82 & $(5.43,6.04)$ & 0.27 & $(0.14,0.40)$ & 5.76 & $(5.47,6.04)$ & 0.52 & $(0.30,1.08)$ & 5.49 & $(4.62,5.94)$ \\
\hline
\end{tabular}

Note. - Surface brightness units: $10^{-12} \mathrm{erg} \mathrm{cm}^{-2} \mathrm{~s}^{-1} \mathrm{deg}^{-2}$. The values in parentheses are the $90 \%$ confidence intervals.

${ }^{a}$ The $\mathrm{X}$ or $\mathrm{S}$ in parentheses indicates whether the shadow was observed with XMM-Newton or Suzaku, respectively.

models, the temperature and normalization of the foreground component are shown in Columns 2 and 3, respectively. In all cases, the halo temperature, $T_{\mathrm{h}}$, and emission measure, $\mathcal{E}_{\mathrm{h}}$, are shown in Columns 8 and 9 , respectively. Column 10 contains $\chi^{2}$ and the number of degrees of freedom (dof) for each fit. The intrinsic surface brightnesses of the foreground $(0.4-1.0 \mathrm{keV})$ and halo $(0.5-2.0 \mathrm{keV})$ components of our models, $S_{\mathrm{fg}}^{0.4-1.0}$ and $S_{\mathrm{h}}^{0.5-2.0}$, respectively, are shown in Table 3 . Note that, because the foreground components are generally softer than the halo components, the foreground surface brightnesses are for a lower energy band than the halo surface brightnesses.

Figures 3 [5 show the observed on- and off-shadow spectra along with the best fit spectral models obtained with the C14-SWCX, ACX-SWCX, and LB spectral models, respectively (assuming Aspl09 abundances). In general the fits are good, typically with $\chi_{\nu}^{2} \approx 1.0-1.4$, where $\chi_{\nu}^{2}$ is the reduced $\chi^{2}$.

The foreground results are plotted in Figure 6. For each foreground model, we show a measure of the foreground hardness (the O VII/C VI ratio for C14-SWCX, the temperature for ACX-SWCX and LB) against the $0.4-1.0 \mathrm{keV}$ surface brightness. The foreground surface brightnesses span more than a order of magnitude, from $\sim 10^{-13}$ to $\sim 4 \times 10^{-12} \mathrm{erg} \mathrm{cm}^{-2} \mathrm{~s}^{-1} \mathrm{deg}^{-2}$. There is a slight tendency for the brighter foreground models to be harder (although this correlation is not statistically significant). This is almost entirely due to the
O VII K $\alpha$ emission - a harder foreground model means brighter O VII emission, which means a higher total surface brightness. The various foreground models do not always yield consistent results - we will examine this further in Section 4.1

Figure 7 shows the halo results obtained using our reference model. In addition, we show results for G225-66 (Henlev et al. 2015a). ${ }^{11}$ The measured temperatures range from $1.6 \times 10^{6}$ to $2.3 \times 10^{6} \mathrm{~K}$, with detected emission measures in the range $(5-17) \times 10^{-3} \mathrm{~cm}^{-6} \mathrm{pc}$ and intrinsic $0.5-2.0 \mathrm{keV}$ surface brightness in the range $(1-7) \times$ $10^{-12} \mathrm{erg} \mathrm{cm}^{-2} \mathrm{~s}^{-1} \mathrm{deg}^{-2}$. With this model, we do not detect halo emission in the direction of MBM 16, consistent with the result previously reported by Ursino et al. (2014).

Our sample includes two shadows - the filament and MBM 20 - that have been observed more than once, albeit with different observatories. For each shadow, while the foreground emission may be different in the two observations, ${ }^{12}$ due to changes in the SWCX emission, we

11 Henley et al. (2015a) used AG89 abundances in their analysis of G225-66. As they discussed, because the halo emission in the XMM-Newton/Suzaku band is dominated by oxygen K $\alpha$ emission, the best fit halo emission measure is approximately inversely proportional to the assumed oxygen abundance. We must allow for this if we wish to fairly compare the emission measures obtained with different abundance tables. Therefore, in Figure 7 we have multiplied the emission measures obtained using AG89 abundances by the ratio of the AG89 and Aspl09 oxygen abundances, $\left(8.51 \times 10^{-4}\right) /\left(4.90 \times 10^{-4}\right)=1.74$.

12 For brevity, from here on we will use the word "observation" 

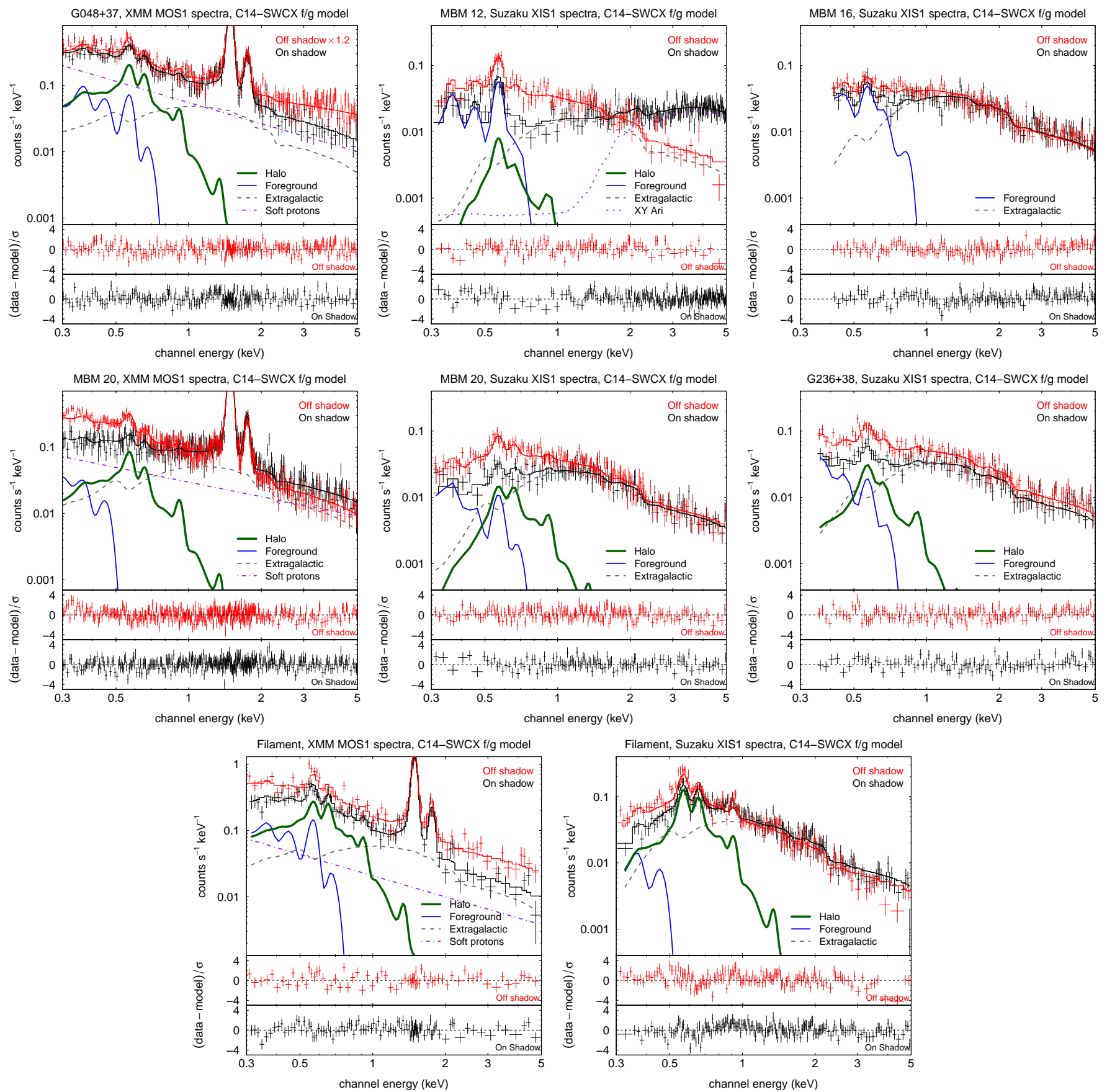

Figure 3. Observed on- and off-shadow spectra for each of our shadows, with the best-fit models obtained using the C14-SWCX foreground model, assuming Aspl09 abundances. For the XMM-Newton observations we show the MOS1 spectra, while for the Suzaku observations we show the XIS1 spectra. For plotting purposes only, the data have been regrouped such that each bin has a signal-to-noise ratio of at least 3. Note that the off-shadow G048+37 data points have been scaled up by $20 \%$ because one of the CCDs was in an anomalous state (Kuntz \& Snowden 2008) and its data could not be used, which also resulted in a relatively brighter soft proton component at low energies than in the on-shadow observation. For the on-shadow spectra, we also show the individual model components, as indicated in the key. Note that, for the XMM-Newton spectra, we show the soft proton component, but not the components representing the instrumental Al and Si lines. For the on-MBM 12 XIS1 spectrum, we show the component representing the residual emission from XY Ari (see Section 3 ). The smaller panels beneath the main panel show the residuals. 

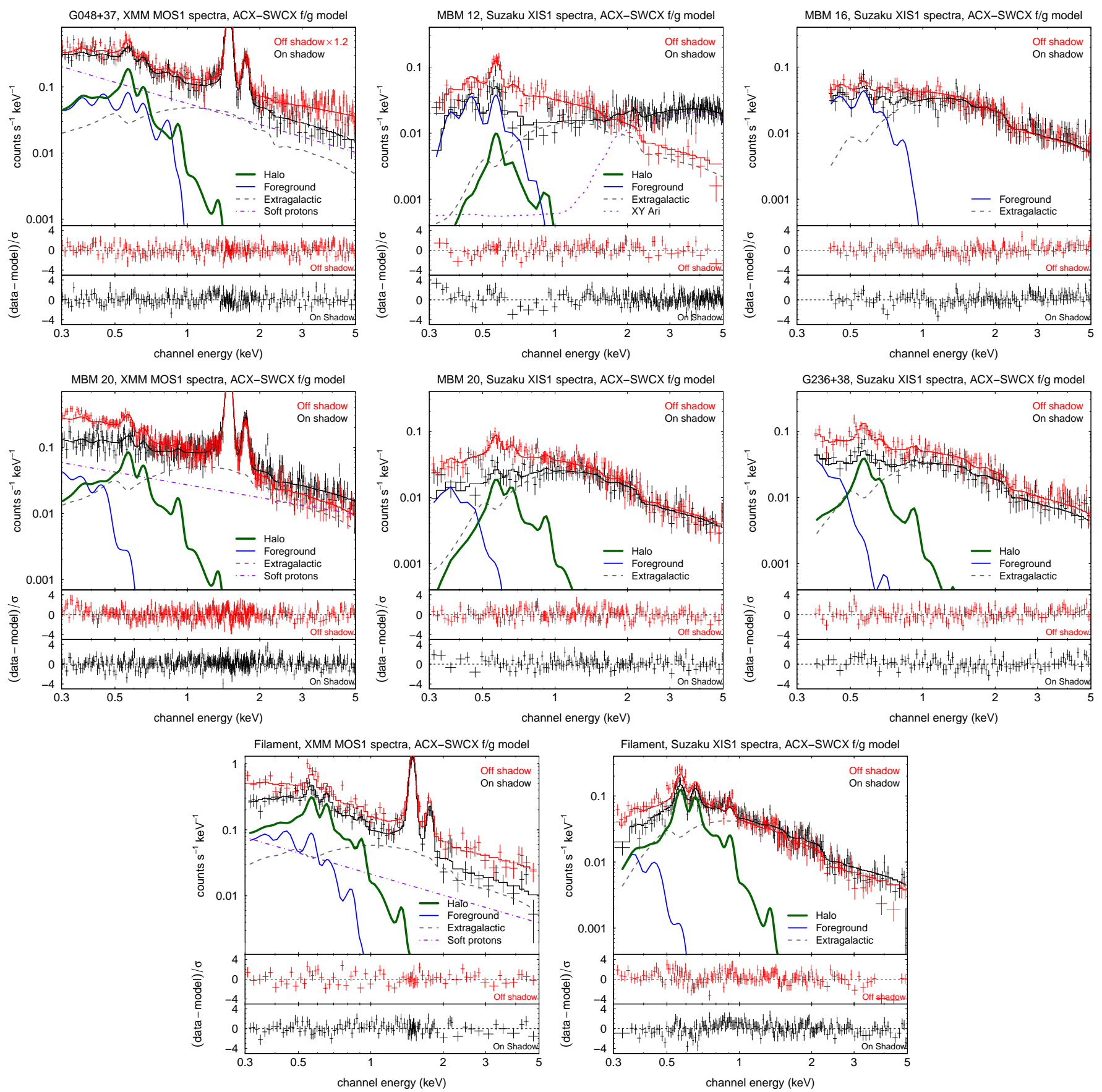

Figure 4. Same as Figure 3 but for the ACX-SWCX foreground model.

should obtain consistent halo measurements from the two observations. With our reference model, we obtain consistent halo temperatures and surface brightnesses from the two MBM 20 observations (green squares), in that the error bars overlap. The errors on the emission measures from these two observations do not quite overlap. For the two filament observations (purple triangles) we obtain consistent halo emission measures, but not temperatures or surface brightnesses.

Our other spectral models yield broadly similar results for the halo-temperatures around $2 \times 10^{6} \mathrm{~K}$, and emis- sion measures and surface brightnesses generally in the above-quoted ranges (after allowing for different oxygen abundances if necessary; see Section 4.2). However, there are differences in the details among some of the models. In the following sections, we will examine the foreground and halo results obtained using different foreground models (Section 4.1) and different abundances (Section 4.2). Finally in this section, we will compare our results with those from previous studies of the same shadows, where available (Section 4.3). 

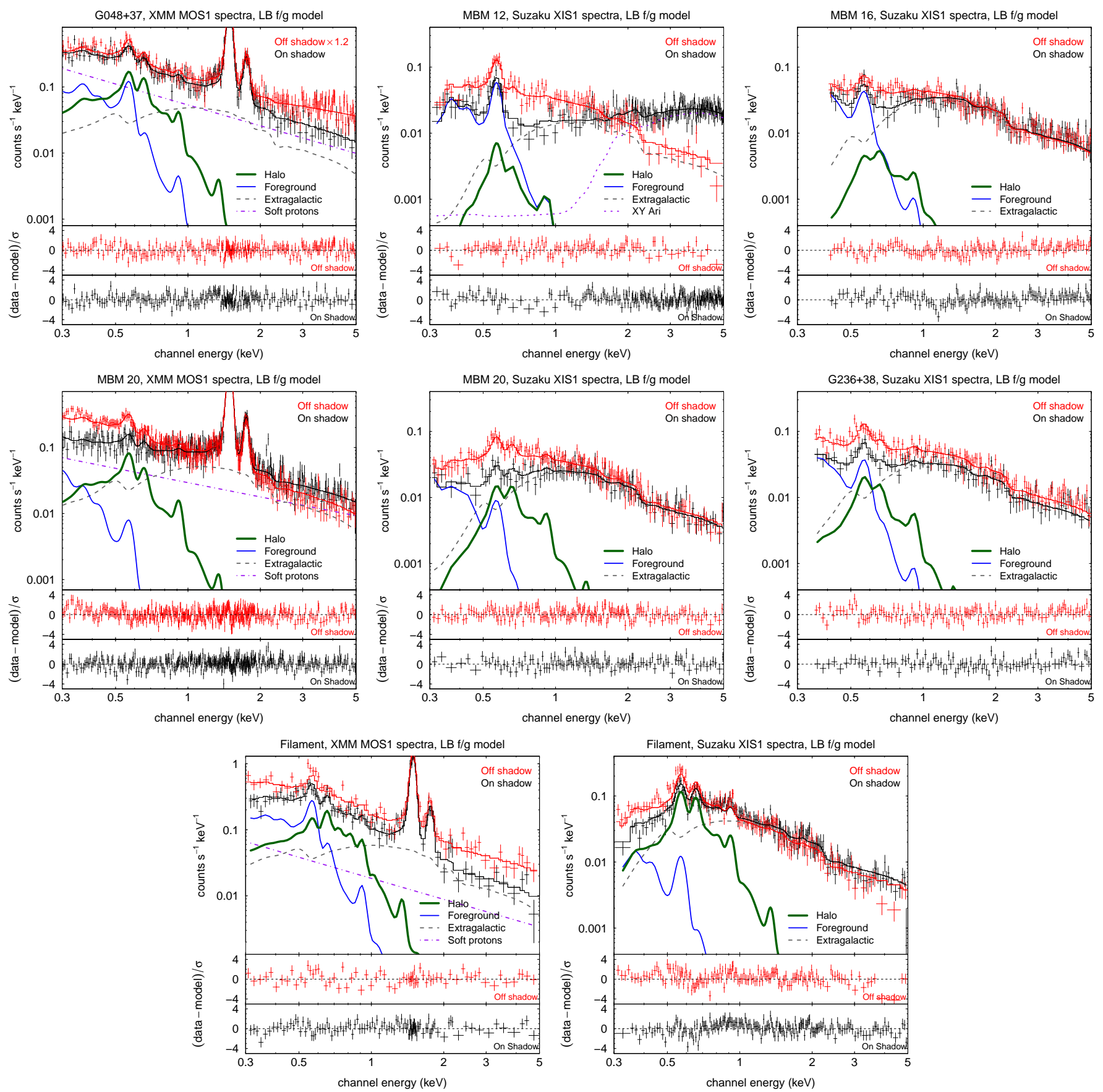

Figure 5. Same as Figure 3 but for the LB foreground model.

\subsection{Results Obtained with Different Foreground Models}

Figure 8 compares the foreground and halo results obtained using our different foreground models. In general, the various foreground models yield consistent foreground surface brightnesses (Figure 8(a)). However, in a couple of cases, the ACX-SWCX model yields a lower best-fit foreground surface brightness than the C14-SWCX model (specifically G236+38 and the Suzaku observation of MBM 20, although it should be noted that the differences are not statistically significant). If we compare the relevant panels in Figure 3 (C14-SWCX) and Figure 4 (ACX-SWCX), we see that these lower
ACX-SWCX surface brightnesses are due to the absence of foreground $\mathrm{O}$ VII $\mathrm{K} \alpha$ emission at $\sim 0.57 \mathrm{keV}$ in the ACX-SWCX models. The foreground O VII $\mathrm{K} \alpha$ emission is also fainter with the ACX-SWCX model than with the C14-SWCX model for MBM 12 and the $X M M-N e w t o n$ observation of the filament. In contrast, for G048+37, the ACX-SWCX model and C14-SWCX models attribute similar amounts of O VII emission to the foreground, but the foreground O VIII emission is brighter in the ACX-SWCX model.

If using the ACX-SWCX model results in less O VII emission being attributed to the foreground, then naturally more O VII emission is attributed to the halo. 


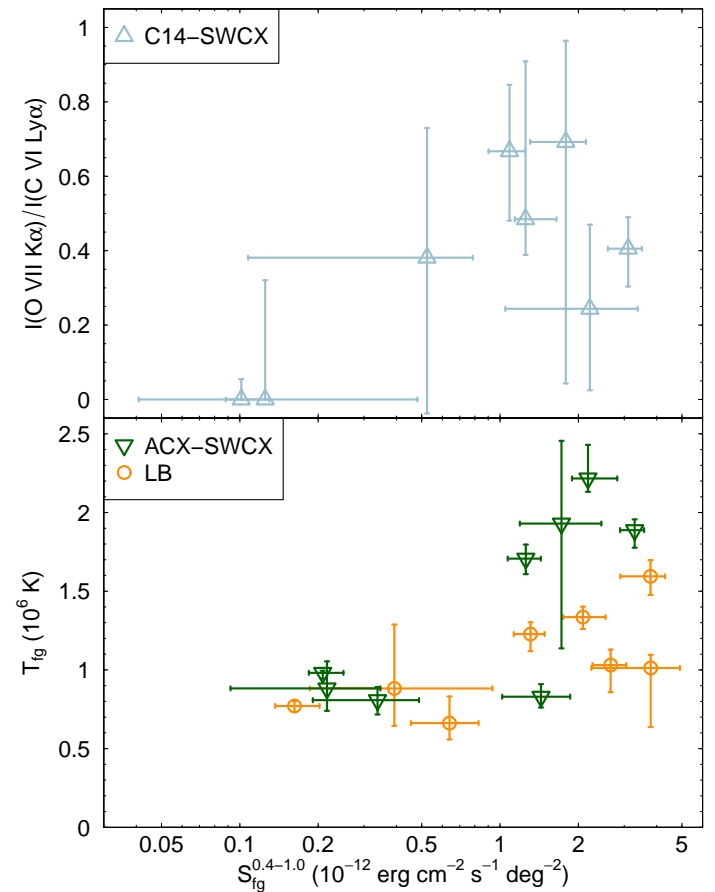

Figure 6. Measures of the foreground hardness against the $0.4-$ $1.0 \mathrm{keV}$ foreground surface brightness. The upper panel shows results for the C14-SWCX model, for which we use the O VII/C VI $\mathrm{K} \alpha$ intensity ratio as our measure of the foreground hardness. The lower panel shows results for the ACX-SWCX (green triangles) and LB (orange circles) models, for which we use the temperature as our measure of the foreground hardness. All results were obtained using Aspl09 abundances.

The amount of O VIII emission attributed to the halo, meanwhile, is typically unaffected, and so the halo $\mathrm{O} \mathrm{VII} / \mathrm{O} \mathrm{VIII}$ ratio tends to increase. In the case of G048+37, attributing more O VIII to the foreground results in less being attributed to the halo, which also increases the halo O VII/O VIII ratio. Thus, in some cases, we find that the ACX-SWCX foreground model yields lower halo temperatures than the C14-SWCX model (Figure 8 (b)). Correspondingly, there is a slight tendency for the ACX-SWCX model to yield higher halo emission measures (Figure 8(c)). This is because the $\mathrm{O}$ VIII emissivity is an increasing function of temperature around $T=2 \times 10^{6} \mathrm{~K}$. If the halo O VIII emission is unaffected by a change in the foreground model, a decrease in the best-fit halo temperature must be compensated by an increase in the halo emission measure.

In general the ACX-SWCX foreground model yields similar halo surface brightnesses to the C14-SWCX model (Figure 8 $(\mathrm{d})$ ), because any changes in the foreground surface brightness are typically small compared with the halo surface brightness. However, for G048+37 $(\mathrm{G} 236+38)$ the ACX-SWCX foreground surface bright-

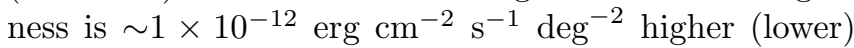
than that obtained with the C14-SWCX model, which is compensated for by a corresponding decrease (increase) in the halo surface brightness.

For seven out of eight sight lines, using a LB foreground model resulted in a larger foreground surface brightness than did using a C14-SWCX foreground model. For six of these seven sight lines the LB model also yielded a lower halo surface brightness. These differences, how-

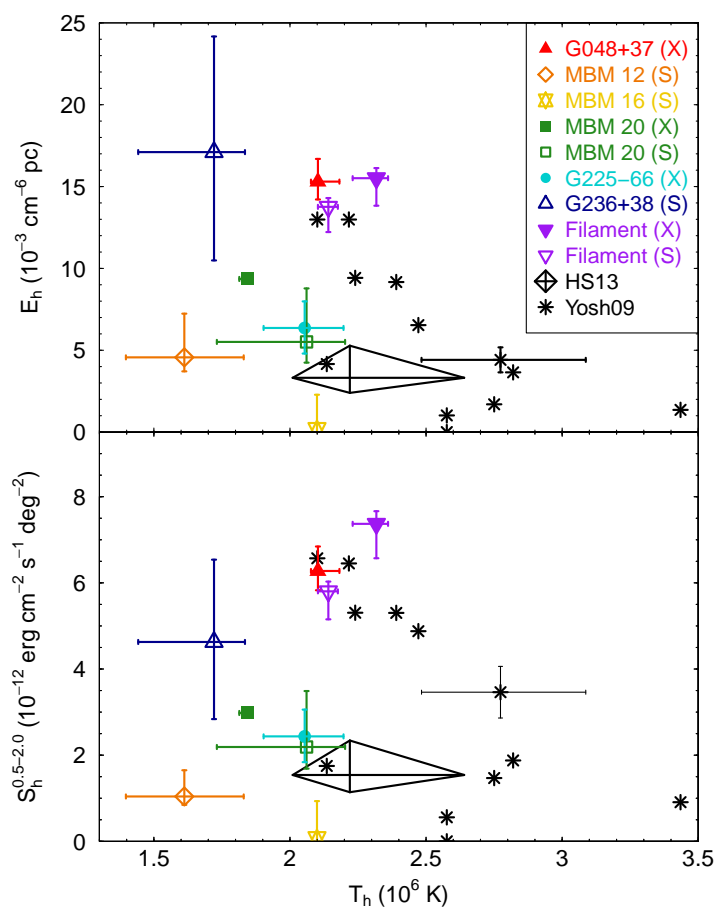

Figure 7. Halo emission measure, $\mathcal{E}_{\mathrm{h}}$, and intrinsic $0.5-2.0 \mathrm{keV}$ surface brightness, $S_{\mathrm{h}}^{0.5-2.0}$, against halo temperature, $T_{\mathrm{h}}$, obtained with our reference model (C14-SWCX foreground model with Aspl09 abundances). Each observation is plotted with its own symbol-see key for details (note that XMM-Newton (X) and Suzaku (S) results are plotted with filled and open symbols, respectively, and that different observations of the same shadow are plotted in the same color). In addition, we show results for G225-66 obtained using the C14-SWCX model (Henley et al. 2015a; note that we have rescaled their emission measure, as they assumed AG89 abundances; see footnote 11). The large black crossed diamonds indicate the median values and the lower and upper quartiles from HS13's XMM-Newton survey of the halo emission, for sight lines on which such emission was detected (first row of their Table 2). The black stars indicate results from the Yosh09 Suzaku study of the halo (from their Table 6; we calculated the surface brightnesses using their best-fit temperature and emission measures, taking into account non-solar abundance ratios if necessary). To reduce clutter, we only show errors for a single, typical Yosh09 observation. As with G225-66, we have rescaled the HS13 and Yosh09 emission measures, because these results were also obtained using AG89 abundances. We will compare our results with those from these other studies in Section 5.4 .1

ever, are generally small (typically $\lesssim 0.2$ dex for the foreground surface brightness) and not statistically significant considering the sizes of the error bars. In general, the LB foreground models are similar in overall spectral shape to the C14-SWCX models. As a result, the LB and C14-SWCX models generally yield similar halo results. However, there are exceptions to this general rule, which occur when the foreground surface brightness is relatively bright. These exceptions include G236+38 and the XMM-Newton observation of the filament. The LB model attributes more O VII emission to the foreground than the C14-SWCX model for these observations (compare the relevant panels in Figures 3 and 5 ). As a result, for these sight lines the LB model yields a higher foreground surface brightness (by a factor of $\sim 2$ ), a higher halo temperature (because less O VII emission is attributed to the halo), a lower halo emission measure, and a lower halo surface brightness (although for G236+38 the differences are not statistically significant). 


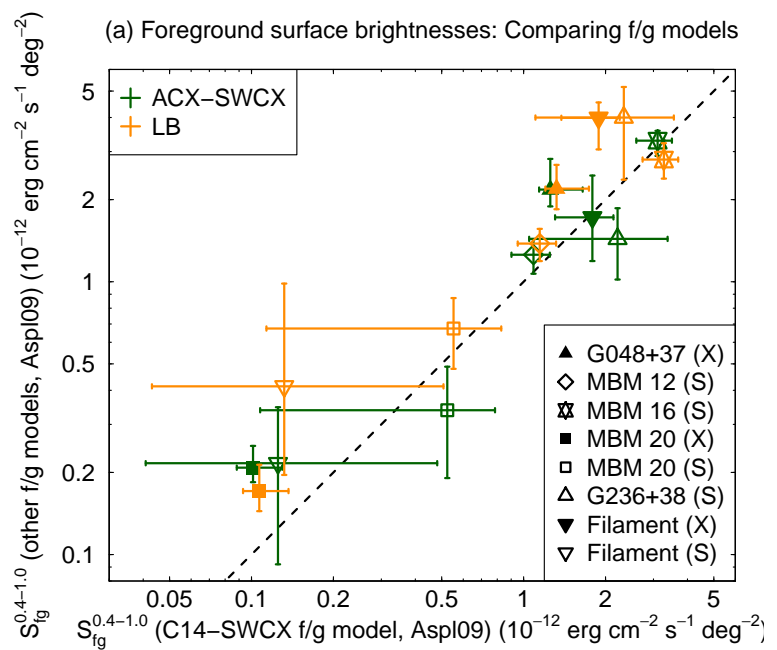

(c) Halo emission measures: Comparing f/g models

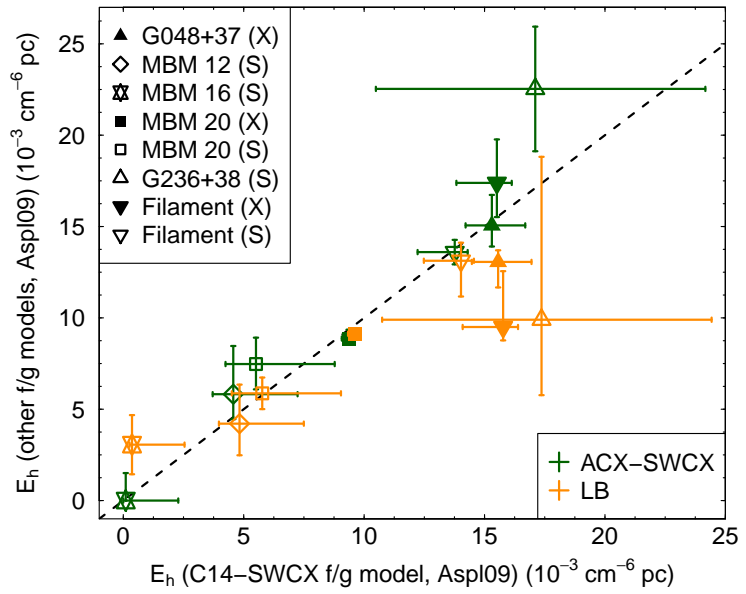

(b) Halo temperatures: Comparing f/g models
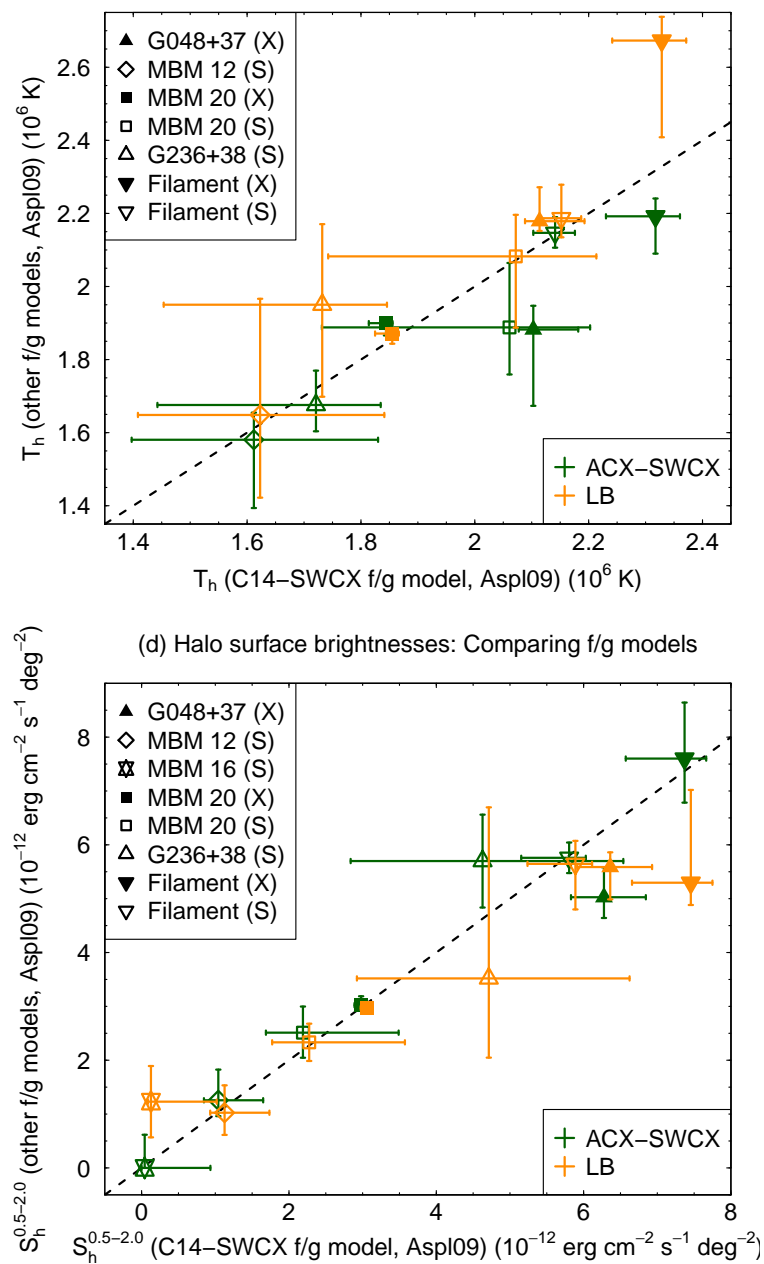

Figure 8. Comparison of (a) the foreground 0.4-1.0 keV surface brightnesses, (b) the halo temperatures, (c) the halo emission measures, and $(\mathrm{d})$ the halo intrinsic $0.5-2.0 \mathrm{keV}$ surface brightnesses obtained using different foreground models. Each panel compares the values obtained using the ACX-SWCX and LB foreground models (green and orange symbols, respectively) with those obtained using our reference model (C14-SWCX foreground model). The various shadows' results are plotted with different symbols, using the same symbols (but not colors) used in Figure 7 All results were obtained using Aspl09 abundances. To avoid overlapping error bars, we have shifted the points comparing the LB results with the C14-SWCX results upward and to the right (by $1 \%$ of the range of each horizontal axis). Note that panel (a) has logarithmic axes, while the other panels have linear axes. Note also that the halo temperature plot (panel (b)) does not include MBM 16, as the halo temperature was not a free parameter for this shadow (see Section 3 ). In all panels, the dashed line indicates equality.

Another exception is MBM 16-with the LB model we detect halo emission, which we do not with the C14SWCX model. This is likely because, in order to fit the O VII emission and the softer emission, the LB component's temperature is such that there is little emission attributed to the foreground above $\sim 0.6 \mathrm{keV}$. Therefore, non-zero halo emission is needed to adequately fit the spectra in this energy range. In contrast, the C14SWCX model can fit the emission above $\sim 0.6 \mathrm{keV}$ with the foreground component, leading to a better fit (note that MBM 16 is the only observation for which we detect foreground O VIII emission with the C14-SWCX model).

In summary, for most sight lines the different foreground models yield consistent results, within the error bars. However, the ACX-SWCX model has a tendency to attribute less $\mathrm{O}$ VII emission to the foreground than the other foreground models. We will discuss why this is the case in Section 5.1, but we will note here that it is likely an artifact of the assumption that the solar wind ion distribution is described by a single temperature. Since this is not a good description of the true solar wind ion distribution (von Steiger et al. 2000), the ACX-SWCX results may be unreliable. In addition, for a few sight lines on which the foreground surface brightness is relatively bright the LB and C14-SWCX foreground models yield discrepant results. We will discuss the implications of this for the halo results in Section 5.4.2.

\subsection{Results Obtained with Different Abundance Tables}

Figure 9 compares the foreground and halo results obtained using Aspl09 and AG89 abundances. The AG89 abundances result in systematically larger foreground surface brightnesses than the Aspl09 abundances (Figure 9(a)). This difference can be understood, at least qualitatively, from the fact that the AG89 carbon, nitrogen, and oxygen abundances are higher than the Aspl09 abundances (by $0.13,0.22$, and 0.24 dex, respectively), resulting in larger photoelectric absorption cross-sections 
(a) Foreground surface brightnesses: AG89 vs. Aspl09

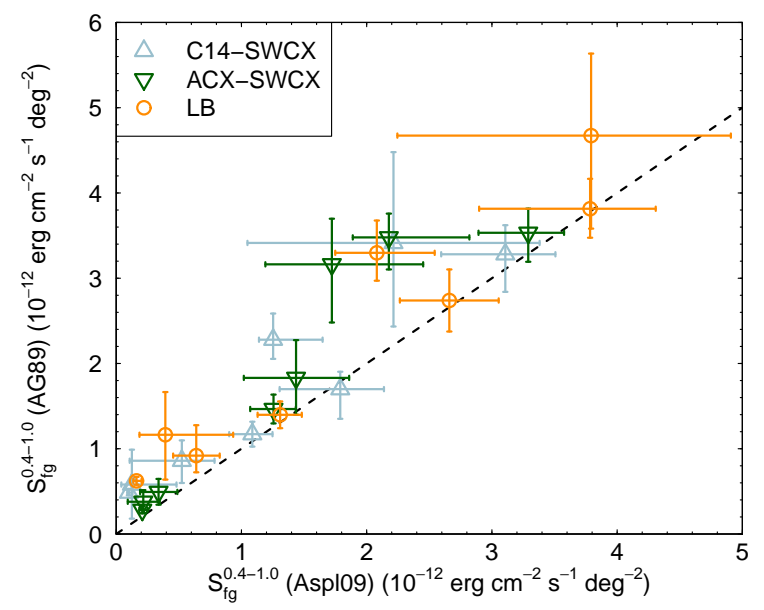

(c) Halo emission measures: AG89 vs. Aspl09

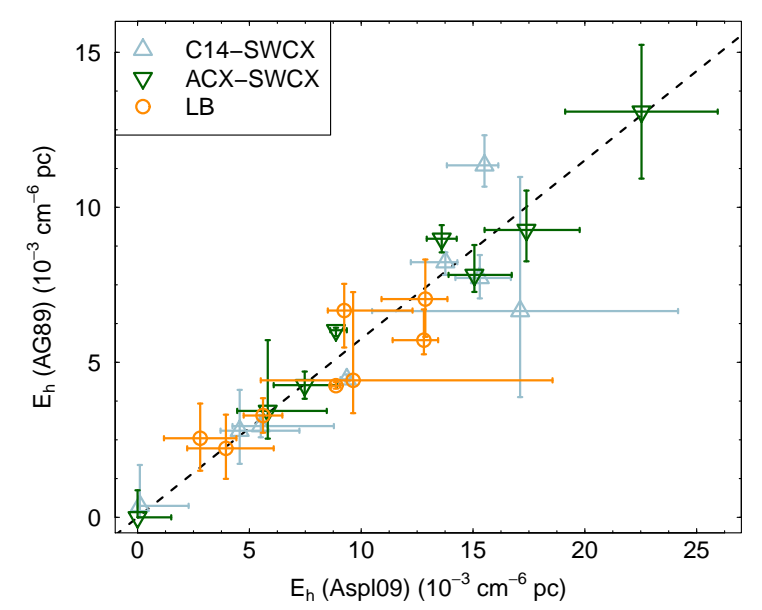

(b) Halo temperatures: AG89 vs. Aspl09

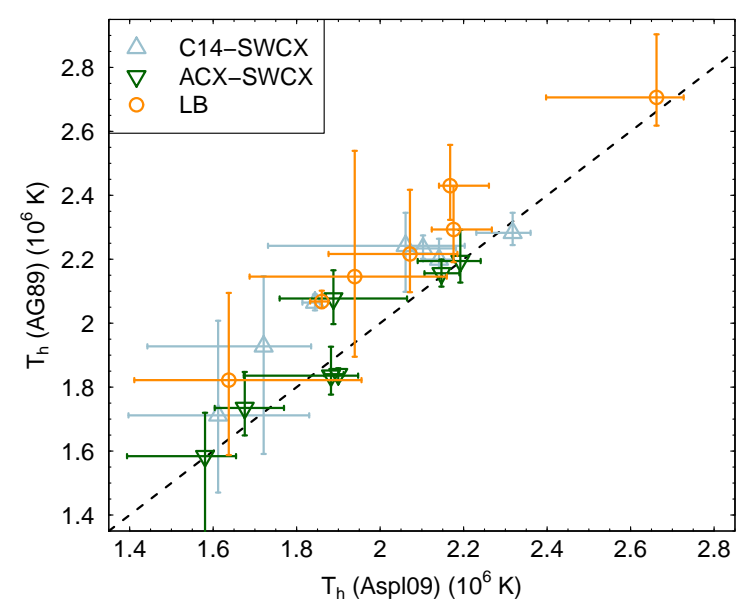

(d) Halo surface brightnesses: AG89 vs. Aspl09

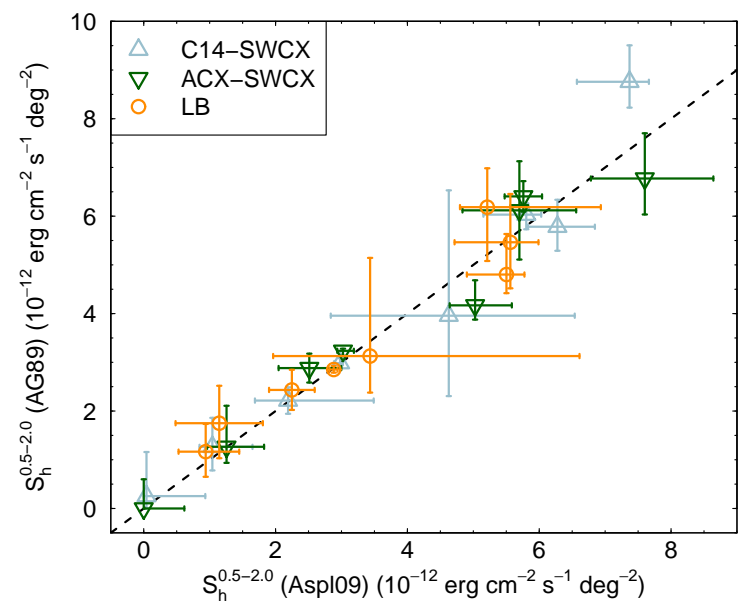

Figure 9. Comparison of (a) the foreground 0.4-1.0 keV surface brightnesses, (b) the halo temperatures, (c) the halo emission measures, and $(\mathrm{d})$ the halo intrinsic $0.5-2.0 \mathrm{keV}$ surface brightnesses obtained using AG89 (ordinates) and Aspl09 (abscissae) abundances, for each of our three foreground models (see key). Note that the halo temperature plot (panel (b)) does not include MBM 16, as the halo temperature was not a free parameter for this shadow (see Section 3). In general, the dashed lines indicate equality. However, in the halo emission measure plot (panel (c)), the gradient of the dashed line is equal to the ratio of the Aspl09 and AG89 oxygen abundances (0.576), rather than 1 (see footnote 15).

at the emission energies being considered. Increasing the absorption cross-section increases the foreground surface brightness inferred from the observations, as we shall now demonstrate.

If, for a given energy band, the foreground, halo, and extragalactic surface brightnesses are $S_{\mathrm{fg}}, S_{\mathrm{h}}$, and $S_{\mathrm{eg}}$, respectively, then the observed on- and off-shadow surface brightnesses are

$$
S_{\mathrm{on}}=S_{\mathrm{fg}}+S_{\mathrm{h}} e^{-\sigma N_{\mathrm{H}, \text { on }}}+S_{\mathrm{eg}} e^{-\sigma N_{\mathrm{H}, \mathrm{on}}}
$$

and

$$
S_{\text {off }}=S_{\mathrm{fg}}+S_{\mathrm{h}} e^{-\sigma N_{\mathrm{H}, \mathrm{off}}}+S_{\mathrm{eg}} e^{-\sigma N_{\mathrm{H}, \mathrm{off}}},
$$

respectively, where $\sigma$ is the band-averaged absorption cross-section, and $N_{\mathrm{H} \text {,on }}$ and $N_{\mathrm{H} \text {,off }}$ are the on- and offshadow hydrogen column densities, respectively. ${ }^{13}$ Solv-

13 Note that, because we are not considering absorption at a single energy, $\sigma$ will be a function of $N_{\mathrm{H}}$ and of the assumed background spectrum (Snowden et al. 1994). However, Figure 4(b) in Snowden et al. (1994) shows that, for a halo temperature of $\gtrsim 2 \times 10^{6} \mathrm{~K}$, the band-averaged absorption cross-section for the ing the above equations for $S_{\mathrm{fg}}$, we obtain

$$
S_{\mathrm{fg}}=\frac{S_{\mathrm{on}} e^{\sigma N_{\mathrm{H}, \text { on }}}-S_{\mathrm{off}} e^{\sigma N_{\mathrm{H}, \text { off }}}}{e^{\sigma N_{\mathrm{H}, \text { on }}}-e^{\sigma N_{\mathrm{H}, \text { off }}}}
$$

the derivative of which with respect to $\sigma$ is

$$
\frac{d S_{\mathrm{fg}}}{d \sigma}=\frac{\left(S_{\mathrm{off}}-S_{\mathrm{on}}\right)\left(N_{\mathrm{H}, \text { on }}-N_{\mathrm{H}, \text { off }}\right) e^{\sigma\left(N_{\mathrm{H}, \text { on }}+N_{\mathrm{H}, \text { off }}\right)}}{\left(e^{\sigma N_{\mathrm{H}, \text { on }}}-e^{\sigma N_{\mathrm{H}, \text { off }}}\right)^{2}} .
$$

Because $S_{\text {off }}>S_{\text {on }}$ and $N_{\mathrm{H}, \text { on }}>N_{\mathrm{H}, \mathrm{off}}, d S_{\mathrm{fg}} / d \sigma>$ 0 . Hence, we expect the larger photoelectric absorption cross-sections resulting from the AG89 abundances to lead to larger foreground surface brightnesses being inferred from the observed surface brightnesses $S_{\text {on }}$ and $S_{\text {off }}$. Note, however, that the above analysis doesn't take into account changes in the shapes

ROSAT $3 / 4 \mathrm{keV}$ band does not vary strongly with $N_{\mathrm{H}}$ for the range of values considered here (i.e., few $\times 10^{20}$ to few $\times 10^{21} \mathrm{~cm}^{-2}$ ). Therefore, we do not expect $\sigma$ for the $0.4-1.0 \mathrm{keV}$ band to vary strongly over this $N_{\mathrm{H}}$ range. 
(a) Foreground surface brightnesses: Wilm00 vs. Aspl09

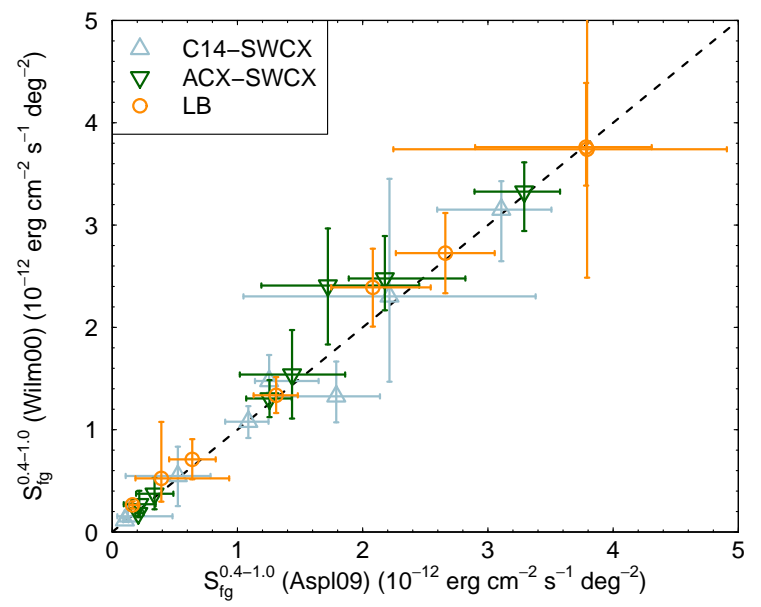

(c) Halo emission measures: Wilm00 vs. Aspl09

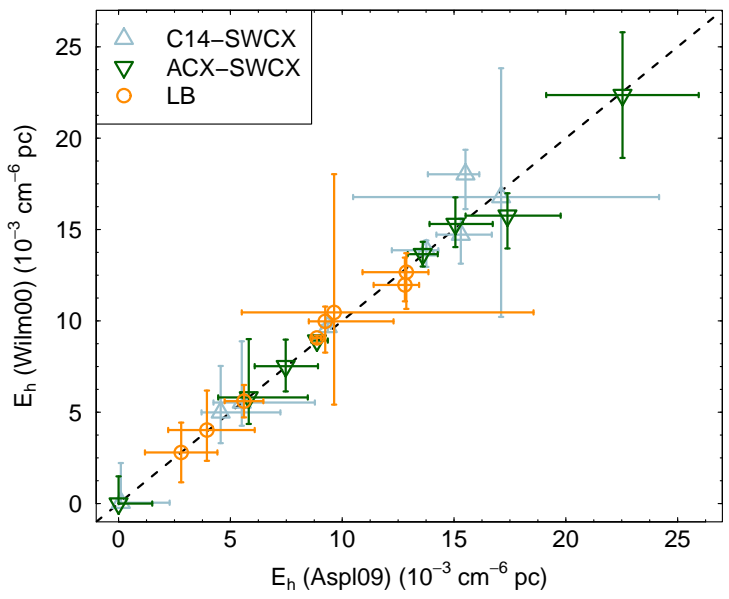

(b) Halo temperatures: Wilm00 vs. Aspl09

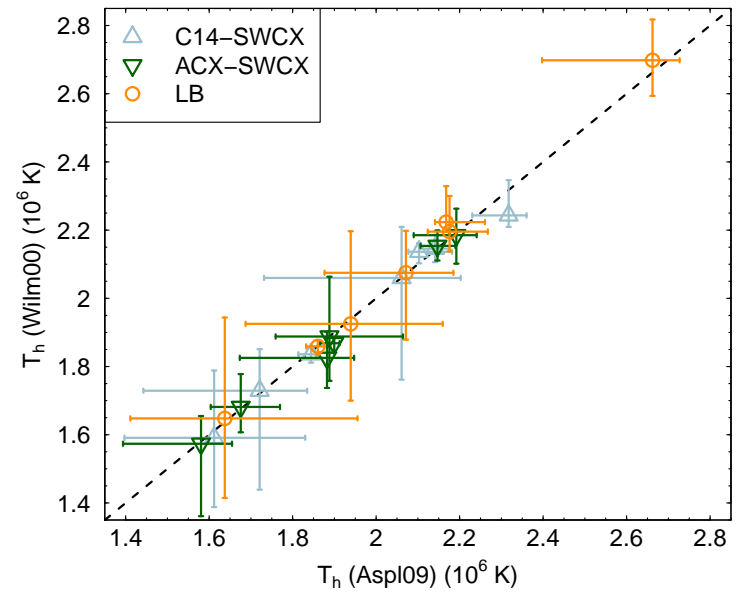

(d) Halo surface brightnesses: Wilm00 vs. Aspl09

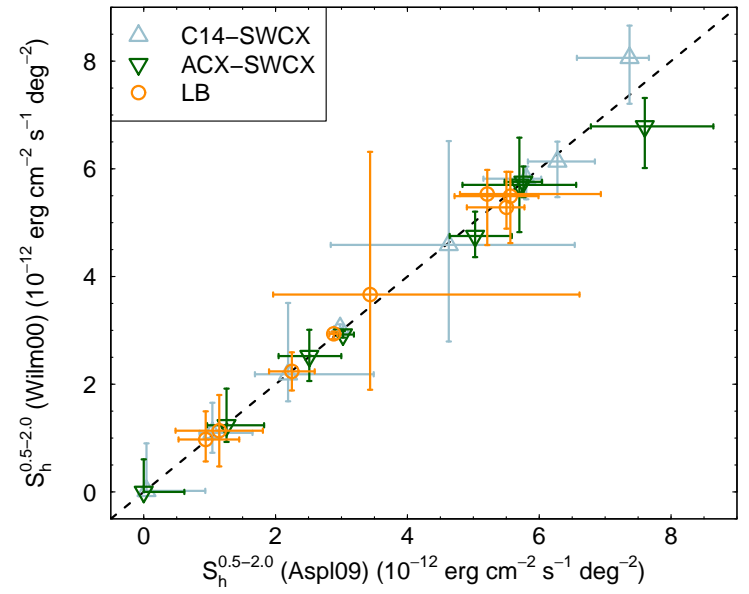

Figure 10. Same as Figure 9, but comparing results obtained with Wilm00 (ordinates) and Aspl09 (abscissae) abundances. However, unlike Figure 9 the gradient of the dashed line in panel (c) is 1, as the oxygen abundance is the same in the Wilm00 and Aspl09 abundance tables.

of the spectral components that will also occur when the abundances are changed. In practice, the differences between the foreground $0.4-1.0 \mathrm{keV}$ surface brightnesses measured using AG89 and Aspl09 abundances (Table 3) imply values of $d S_{\mathrm{fg}} / d \sigma$ that are typically $\sim 2-3$ times smaller than the values predicted by Equation (4) (the predicted values are typically $\left.\sim 10^{-12} \mathrm{erg} \mathrm{cm}^{-2} \mathrm{~s}^{-1} \mathrm{deg}^{-2}\left(10^{-22} \mathrm{~cm}^{2}\right)^{-1}\right){ }^{14}$

As the foreground emission is generally softer than the halo emission, increasing the foreground surface brightness decreases the softer halo emission relative to the harder halo emission. As a result, the AG89 abundances generally yield higher halo temperatures than the Aspl09 abundances (Figure 9(b)), particularly for the C14-SWCX and LB foreground models. Associated with these higher halo temperatures are lower halo emission measures (lower than can be accounted for by the difference in oxygen abundance ${ }^{15}$ Figure $\left.9(\mathrm{c})\right)$. Despite these

14 For these calculations we used cross sections of $5.15 \times 10^{-22}$ and $6.75 \times 10^{-22} \mathrm{~cm}^{2}$ for Aspl09 and AG89 abundances, respectively, calculated for a $2.1 \times 10^{6} \mathrm{~K}$ plasma observed through an absorbing column density of $10^{21} \mathrm{~cm}^{-2}$.

${ }^{15}$ As noted in footnote 11 we must account for differences in the differences, AG89 and Aspl09 abundances generally yield similar halo surface brightnesses (Figure 9(d)). The reason is likely the same as that mentioned in Section 4.1 the changes in the foreground surface brightness resulting from changing the assumed abundances are generally small relative to the total halo surface brightness.

Figure [10 is similar to Figure 9] but compares the Wilm00 results with the Aspl09 results. In general, the two sets of results are in good agreement, which is likely mainly due to the similarity of the carbon, nitrogen, and oxygen abundances, and hence of the photoelectric absorption cross-sections.

In summary, while the Wilm00 and Aspl09 abundance tables generally yield very similar results, there are systematic differences between these results and those obtained using AG89 abundances. These differences result, at least in part, from differences in the photoelectric ab-

oxygen abundance when comparing emission measures obtained using different abundance tables. Therefore, in the halo emission measure plot (Figure 9] c)), the gradient of the dashed line is equal to the ratio of the Aspl09 and AG89 oxygen abundances (0.576). Setting the line gradient to this value helps us identify differences in the halo emission measures that are not simply due to the difference in the assumed oxygen abundance. 

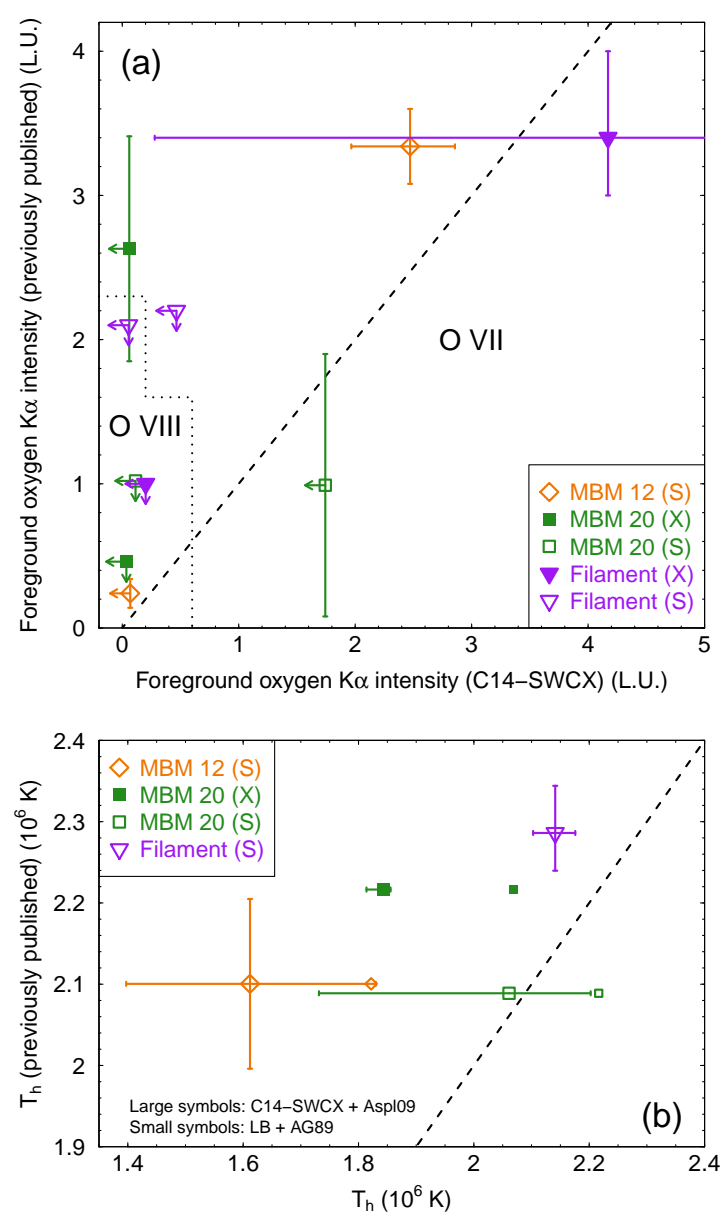

Figure 11. Comparison of our measurements of (a) the foreground $\mathrm{O}$ VII and $\mathrm{O}$ VIII $\mathrm{K} \alpha$ intensities and (b) the halo temperature with measurements from previous shadowing studies. In panel (a), the dotted line separates the O VIII results (lower left region) from the O VII results. The symbols and colors for the various shadows match those used in Figure 7 The published results for MBM 12 and the XMM-Newton and Suzaku observations of MBM 20 are from Smith et al. (2007), Galeazzi et al. (2007), and Gupta et al. (2009), respectively. The published oxygen results for the XMM-Newton observation of the filament are from Henley et al. (2007, note that they did not examine a $1 T$ halo model), and the published results for the Suzaku observation of the filament are from Henlev \& Shelton (2008, oxygen intensities) and Lei et al. (2009, halo temperature). Note that the published oxygen measurements are summarized in Table 4 of Gupta et al. (2009). Our results are primarily from our reference model (C14SWCX foreground and Aspl09 abundances). However, for shadows previously analyzed with AG89 abundances (MBM 12 and MBM 20), we also show in panel (b) the temperatures we obtained using the LB foreground model with AG89 abundances, using smaller symbols without error bars. The dashed lines indicate equality.

sorption cross-sections. Since the AG89 carbon, nitrogen, and oxygen abundances are likely too high for the sun and for the interstellar medium, the results obtained with this abundance table are likely unreliable.

\subsection{Comparison with Previous Shadowing Studies}

A number of the shadows in our sample have previously been analyzed. Here, we compare the results from these earlier studies with our results.

Figure 11(a) compares the foreground O VII and $\mathrm{O}$ VIII $\mathrm{K} \alpha$ intensities from our reference model with the previously published values. In general the results are consistent, considering the large scatter and error bars. However, we typically obtain tighter upper limits on the foreground O VIII Ly $\alpha$ intensity. This is likely because our results were obtained directly from the spectral fitting, whereas most of the previously published results were obtained by measuring the total O VIII intensities in the on- and off-shadow spectra independently, and then using an equation similar to Equation (3) to calculate the foreground intensity. (This also accounts for our tighter upper limit on the foreground O VII intensity from the Suzaku observation of the filament.) In addition, our C14-SWCX foreground model includes emission from $\mathrm{O}$ VII $\mathrm{K} \beta$ (at $0.666 \mathrm{keV}$, with an intensity $\sim 7 \%$ of that from $\mathrm{K} \alpha$ ), which would tend to reduce the intensity attributed to the foreground O VIII (at $0.653 \mathrm{keV}$ ).

For MBM 12 we obtain a lower foreground O VII $\mathrm{K} \alpha$ than Smith et al. (2007). This is due, at least in part, to our using a lower on-cloud column density $\left(3.22 \times 10^{21}\right.$ versus $\left.4 \times 10^{21} \mathrm{~cm}^{-2}\right)$ and to our assuming Aspl09 instead of AG89 abundances, lowering the absorption cross-section at $0.57 \mathrm{keV}\left(6.8 \times 10^{-22}\right.$ versus $\left.9.4 \times 10^{-22} \mathrm{~cm}^{2}\right)$. Both of these changes increase the transmissivity of the cloud to background O VII emission, from $2 \%$ to $11 \%$. Smith et al. (2007) assumed that the contribution of the halo emission to the on-cloud O VII emission was negligible, whereas in our model $\sim 10 \%$ of the on-cloud O VII emission is due to halo emission leaking through the cloud (see the MBM 12 plot in Figure 3).

For the MBM 20 observations, we obtain upper limits on the foreground $\mathrm{O}$ VII $\mathrm{K} \alpha$ intensity, whereas Galeazzi et al. (2007) and Gupta et al. (2009) quote detections. However, the reported detection from the Suzaku observation (Gupta et al. 2009) is borderlineif the quoted error is $1 \sigma$, then at the $90 \%$ level which we use for our errors, the line was not detected. Note also that Gupta et al. (2009) and we obtained almost identical best-fit foreground $\mathrm{O}$ VII $\mathrm{K} \alpha$ intensities from this observation (0.99 and 0.97 L.U., respectively).

For the XMM-Newton observation of MBM 20, we obtain a very tight upper limit on the foreground O VII intensity (0.06 L.U. at the $90 \%$ level). This is likely because the halo model is very tightly constrained (note from the solid green squares in Figure 7 that this observation has the smallest uncertainties on the halo measurements) and can adequately explain the observed O VII emission alone, leaving little room for any foreground O VII emission. We checked the possibility that this tight upper limit on the foreground O VII intensity is an artifact of our assuming a $1 T$ halo model, meaning that the halo spectrum may be artificially tightly constrained. However, when we added a second thermal plasma component to our halo model, we obtained an upper limit on the foreground O VII intensity of 0.13 L.U.. While this is not as tight a constraint as with our original model, the foreground O VII intensity is still more tightly constrained for this observation than for any other. Therefore, the tight upper limit on the foreground O VII intensity for the XMM-Newton observation of MBM 20 is not an artifact of our assuming a $1 T$ halo model.

It is unclear exactly why we and Galeazzi et al. (2007) obtained different results from the XMM-Newton observation of MBM 20. However, the difference appears not 
to be due to the fact that Galeazzi et al. (2007) analyzed only the pn data, assumed AG89 abundances, and used lower column densities than us $\left(15.9 \times 10^{20}\right.$ and $0.86 \times 10^{20}$ versus $17.6 \times 10^{20}$ and $2.3 \times 10^{20} \mathrm{~cm}^{-2}$ (Table(1)). If we refit the C14-SWCX model to the pn data only, assuming AG89 abundances and Galeazzi et al.'s column densities, we obtain a foreground O VII intensity of $0.85_{-0.19}^{+0.26}$ L.U. (this is similar to the value in Section (d) of Table2, obtained using the pn and MOS data and the column densities from Table 1). While this is larger than the value obtained with our reference model (shown in Figure 11(a)), it is still smaller than the foreground O VII intensity reported by Galeazzi et al. (2007), $2.63 \pm 0.78$ L.U.. It is possible that the difference arises from the fact that Galeazzi et al. (2007) used a different method from us to calculate the particle background, which may have affected the background-subtracted SXRB spectra, and thus the fit results.

In general, our halo temperatures are in reasonable agreement with (although systematically lower than) the previously published values. The agreement is better if we use a model more similar to those used in most previous studies (an LB foreground model with AG89 abundances, shown by the smaller symbols in Figure 11(b)). The largest temperature discrepancy is for MBM 12 . This is likely due to the higher transmissivity of the cloud to background O VII in our model (see above), resulting in more O VII emission being attributed to the halo, and hence a lower halo O VIII/O VII ratio and a lower halo temperature. For the Suzaku observation of the filament, the $7 \%$ difference between our and Lei et al.'s (2009) halo temperatures is not due to our assuming different column densities (see Section 3) - using Lei et al.'s column densities with our reference model shifts the best-fit temperature by only $1 \%$. Instead, the discrepancy may be due to Lei et al. fixing the foreground model when they fitted a $1 T$ halo model to the Suzaku spectra (see their Table 1). Our halo emission measures (not plotted) are also in reasonable agreement with the previously published values, after accounting for differences in the assumed oxygen abundance (although for three of the four shadowing observations considered here, our best-fit emission measures are larger than the previously published values).

\section{DISCUSSION}

In our spectral analysis, we examined three different foreground models. For some sight lines, these models yielded different results (Section 4.1). Therefore, we begin our discussion by examining the differences among the foreground models, how they are related to the physics of the models, and how we come to favor one foreground model (C14-SWCX) over the others (Section 5.1).

We then discuss our foreground measurements from the C14-SWCX model. Such measurements may be used to test theoretical models of SWCX emission. We therefore compare our measurements with general predictions from heliospheric SWCX emission models (Section [5.2). We also use our measurements to place limits on the emission from the LB (Section 5.3).

Finally, we discuss our halo emission results. In Section 5.4.1 we compare our halo measurements with those from non-shadowing studies of the halo, using XMMNewton (HS13) and Suzaku (Yosh09). In Section 5.4.2.

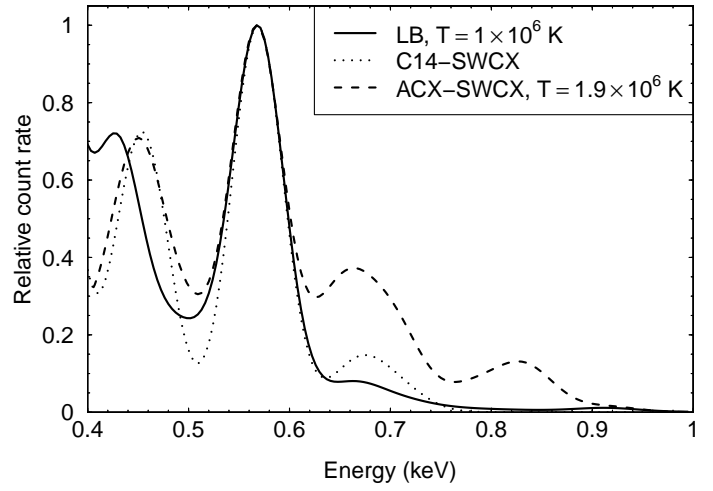

Figure 12. Example model foreground spectra from the LB (solid), C14-SWCX (dotted), and ACX-SWCX (dashed) models, folded through the Suzaku response and normalized to the peak of the O VII emission. The LB model's temperature was fixed at $T=1 \times 10^{6} \mathrm{~K}$, and the other models' parameters were adjusted so that the lower-energy emission $(E \lesssim 0.5 \mathrm{keV})$ was of similar brightness in all three models (see text for details).

we discuss possible uncertainties or biases in our halo measurements in addition to the statistical uncertainties. Finally, we discuss the use of these (and similar) halo measurements in testing models of the Galactic halo emission (Section 5.4.3).

\subsection{Our Preferred Foreground Model}

Here we discuss the differences among the foreground models and how they are related to the physics of the models. This discussion will lead us to pick one foreground model, C14-SWCX, as our preferred model.

We noted in Section 4.1 that, for some sight lines, the ACX-SWCX model attributes less O VII emission to the foreground than the C14-SWCX and LB models, resulting in a softer and fainter foreground. Henlev et al. (2015a) also found this be true for G225-66 (see their Figure 3). Attributing less of this emission to the foreground can affect the measured halo properties.

The above difference between the models arises because the ACX-SWCX foreground model produces more emission above $\sim 0.6 \mathrm{keV}$ relative to the O VII emission than the other foreground models, as shown in Figure 12 . This plot shows model spectra from our three foreground models. We used an LB model with a temperature of $1 \times 10^{6} \mathrm{~K}$ (a typical temperature for such a model; solid line) as a reference. For the C14-SWCX model (dotted line), we disabled the $\mathrm{C} \mathrm{V}$ lines (which lie below the energy range plotted) and the O VIII lines (because the reference LB model in Figure 12 produces negligible O VIII emission). We then adjusted the C VI intensity so that the emission below $\sim 0.5 \mathrm{keV}$ was similar in brightness (relative to $\mathrm{O}$ VII) to that from the LB model. ${ }^{16}$ We adjusted the temperature of the ACX-SWCX model (dashed line) so that its emission below $\sim 0.5 \mathrm{keV}$ was also of similar brightness relative to the O VII emissionthis was achieved at $T=1.9 \times 10^{6} \mathrm{~K}$. Having thus adjusted the model spectra, we can immediately see that the emission above $\sim 0.6 \mathrm{keV}$ is much brighter from the

16 Note that the emission below $\sim 0.45 \mathrm{keV}$ is at different energies in the two models. The emission at $\sim 0.43 \mathrm{keV}$ in the LB model is from N VI K $\alpha$, which is not included in the C14-SWCX model. The emission at $\sim 0.45 \mathrm{keV}$ in the C14-SWCX model is from C VI $\mathrm{Ly} \beta$ and $\mathrm{Ly} \gamma$. 
ACX-SWCX model than from the other two models. The broad feature at $\sim 0.66 \mathrm{keV}$ is mainly due to O VIII Ly $\alpha$, with contributions from $\mathrm{O}$ VII $\mathrm{K} \beta-\mathrm{K} \delta$, while the feature at $\sim 0.83 \mathrm{keV}$ is due to $\mathrm{O}$ VIII Ly $\beta-\mathrm{Ly} \delta$. This difference explains why the ACX-SWCX foreground models that best fit the observations are sometimes softer than the other foreground models: the fitting will not allow the ACX-SWCX models to produce as much foreground $\mathrm{O}$ VII as the other models, as doing so would result in too much higher-energy foreground emission, worsening the fit.

Physically, this difference arises because different ions, with different temperature dependencies, are responsible for the O VII and O VIII emission in the LB and ACX-SWCX models (note that in the C14-SWCX model, the normalizations of the various ions' emission are completely independent, rather than being governed by a single temperature parameter). In the LB model, this emission is due to collisional excitation of $\mathrm{O}^{6+}$ and $\mathrm{O}^{7+}$ ions, respectively, whereas in the ACX-SWCX model, it arises from $\mathrm{O}^{7+}$ and $\mathrm{O}^{8+}$ ions, respectively, undergoing CX reactions with hydrogen and helium. In a CIE plasma at $T=1 \times 10^{6} \mathrm{~K}$ (the temperature of the LB model plotted in Figure 12), $\mathrm{O}^{7+}$ is two orders of magnitude less abundant than $\mathrm{O}^{6+}$ (ATOMDB v2.0.2; Foster et al. 2012), and so there is virtually no $\mathrm{O}$ VIII emission from the LB model. In contrast, at $T=1.9 \times 10^{6} \mathrm{~K}$ (the temperature of the ACX-SWCX model plotted in Figure [12), $\mathrm{O}^{8+}$ is one-ninth as abundant as $\mathrm{O}^{7+}$ (ATOMDB), and so the $\mathrm{O}$ VIII CX emission is not negligible.

In general, when the foreground O VII emission is independent of the foreground emission from other ions (as with the C14-SWCX model), more O VII emission is attributed to the foreground than with the ACX-SWCX model. This suggests that the ACX-SWCX model may not accurately model the foreground emission - the ACX-SWCX models may, in some cases, be artificially soft, which in turn results in underestimated halo temperatures. The inaccuracy of the ACX-SWCX model in this context is likely due to the fact that the ion populations in this model are governed by a single temperature, which is not a good description of the true solar wind ion populations (von Steiger et al.|2000). This shortcoming could in principle be overcome by using multiple independent ACX components to model the foreground. We briefly experimented with a two-component ACX foreground model - one component representing the foreground carbon and nitrogen emission, and one the foreground emission from oxygen and higher- $Z$ elements. We found that the results varied from shadow to shadow. In some cases the ACX-SWCX foreground model was brought more into line with the C14-SWCX model, while in other cases the discrepancies between the ACX-SWCX and C14-SWCX models were even larger. Furthermore, for some observations one of the two ACX components was poorly constrained. Fully exploring multitemperature ACX-SWCX foreground models is beyond the scope of this paper.

In addition, we noted in Section 3 that the ACX model's simple analytical expressions cannot be expected to reproduce the true CX $n$ and $l$ distributions for all relevant reactions. This may result in inaccurate modeling of the higher-order lines relative to the $\mathrm{K} \alpha$ lines. While this may not be important in our spectra, it could be important when modeling higher-quality spectra in which the higher-order lines are clearly seen. Therefore, even in situations where a single ionization temperature is a good description for the ion populations, the ACX model may have to be used with caution.

In contrast to the ACX-SWCX models, the LB foreground models are generally similar in spectral shape and brightness to the C14-SWCX models, and thus generally yield similar halo results. However, a pure-LB foreground model is likely unphysical. ${ }^{17}$ For example, such a model typically implies an LB pressure several times larger than those of the cold interstellar clouds in the vicinity of the sun (e.g., Jenkins 2009). However, the similarity between the C14-SWCX and LB foreground spectra implies that, although unphysical, a $\sim 1 \times 10^{6} \mathrm{~K}$ thermal plasma model may adequately model the foreground emission in a CCD-resolution X-ray spectrum, unless the foreground is particularly bright (see Section 5.4.2). (Unfortunately, this similarity also means that one cannot separate spectroscopically the contributions of LB and SWCX emission to the foreground.)

In summary, because the ACX-SWCX model may be unreliable (due to our assumption of a single ionization temperature) and the LB model is likely unphysical, our preferred foreground model is therefore the C14-SWCX model. It should be noted that this model does not include emission from N VI and N VII-because of the relatively low resolution of the CCD spectrometers at low energies, it is difficult to separate the emission from carbon, nitrogen, and oxygen. However, the relevant nitrogen ions are less abundant in the solar wind than the corresponding carbon and oxygen ions (Schwadron \& Cravens 2000), and so omitting nitrogen should not seriously adversely affect our results. We will use the results from the C14-SWCX model in the following section, when we compare our foreground measurements with some SWCX model predictions.

\subsection{Comparing the Foreground Emission with Heliospheric SWCX Model Predictions}

As noted earlier, our measurements of the foreground emission may be used to test theoretical models of SWCX emission. Generating detailed SWCX predictions for each observation, taking into account contemporaneous solar wind conditions (Koutroumpa et al. 2007, 2011; Gupta et al. 2009), is beyond the scope of this paper. Instead, we will restrict our discussion to the general SWCX predictions of Koutroumpa et al. (2006), who describe how the heliospheric SWCX emission is expected to vary with solar cycle and with ecliptic latitude, $\beta$. In particular, the SWCX emission depends on the path lengths along a given sight line through the slow and fast solar winds - the slow solar wind is more highly ionized than the fast solar wind, and contains more of the ions responsible for SWCX emission in the XMM-Newton/Suzaku band (Schwadron \& Cravens 2000). At solar minimum, the slow solar wind is restricted to within $\pm 20^{\circ}$ of the solar equatorial plane, with the fast solar wind present at higher latitudes, whereas at solar maximum, the solar wind at all lati-

17 Our main reason for including such a model is that it was widely used in earlier shadowing studies. 
Table 4

Sunspot Numbers and Ecliptic Latitudes

\begin{tabular}{cccc}
\hline \hline Shadow & Observatory & $\begin{array}{c}\text { Sunspot } \\
\text { number }\end{array}$ & $\begin{array}{c}\beta^{\mathrm{b}} \\
(\mathrm{deg})\end{array}$ \\
\hline Filament & XMM-Newton & 125.0 & -74.39 \\
MBM 20 & XMM-Newton & 37.8 & -36.20 \\
MBM 12 & Suzaku & 6.5 & +2.60 \\
Filament & Suzaku & 3.6 & -74.41 \\
MBM 20 & Suzaku & 1.8 & -36.23 \\
G236+38 & Suzaku & 47.9 & -12.21 \\
G048+37 & XMM-Newton & 36.7 & +49.23 \\
G225-66 & XMM-Newton & 35.2 & -42.43 \\
MBM 16 & Suzaku & 65.5 & -6.49 \\
\hline \multicolumn{4}{c}{ Note. - In contrast to Table1 the observations } \\
are tabulated in chronological order. \\
a Sunspot data obtained from the National Geophys- \\
ical Data Center (http://www.ngdc.noaa.gov/stp/ \\
space-weather/solar-data/solar-indices/ \\
sunspot-numbers/international/listings/). The tab- \\
ulated values are averaged over 10 d either side of \\
the on-shadow pointing. \\
b Ecliptic latitude of the on-shadow pointing.
\end{tabular}

tudes as approximated as being in a slow solar wind state (Koutroumpa et al. 2006). Therefore, broadly speaking, we expect the SWCX emission to be fainter and softer on high- $\beta$ sight lines at solar minimum, compared with low- $\beta$ sight lines at solar minimum or with all sight lines at solar maximum, because high- $\beta$ sight lines at solar minimum mainly sample the fast solar wind.

To test these expectations, we use the results from the C14-SWCX model (with Aspl09 abundances). We also include results for G225-66 (Henlev et al. 2015a; note that these results were obtained using AG89 abundances). Since we are examining in particular how the SWCX emission depends on solar activity and on ecliptic latitude, we tabulate the contemporaneous sunspot number (a measure a solar activity) and $\beta$ for each of our observations in Table 4 .

Figure [13(a) shows the $0.4-1.0 \mathrm{keV}$ surface brightness of the C14-SWCX foreground model plotted against sunspot number. Note that we use different symbols to denote results from high and low ecliptic latitudes. Among the observations toward higher latitudes, the foreground surface brightness generally increases as the sunspot number increases. This is qualitatively as expected, as the high latitude solar wind changes from a fast state at solar minimum to a slow state at solar maximum.

Of the three observations taken near solar minimum (with sunspot number $<10$ ), the one toward low ecliptic latitude (MBM 12) has the highest foreground surface brightness. Again, this is (qualitatively) as expected - this observation samples only the slow solar wind, whereas the observations toward higher ecliptic latitudes mainly sample the fast solar wind, which produces less CX emission in the XMM-Newton/Suzaku band (Koutroumpa et al. 2006). Similarly, of the five observations with intermediate sunspot numbers $(\sim 35$ 65 ), those toward low ecliptic latitudes tend to have brighter foregrounds than those toward higher latitudes. Whether or not this is as expected depends in part on the large-scale configuration of the solar wind at these intermediate sunspot numbers. Surprisingly, one of these observations (G225-66) yielded

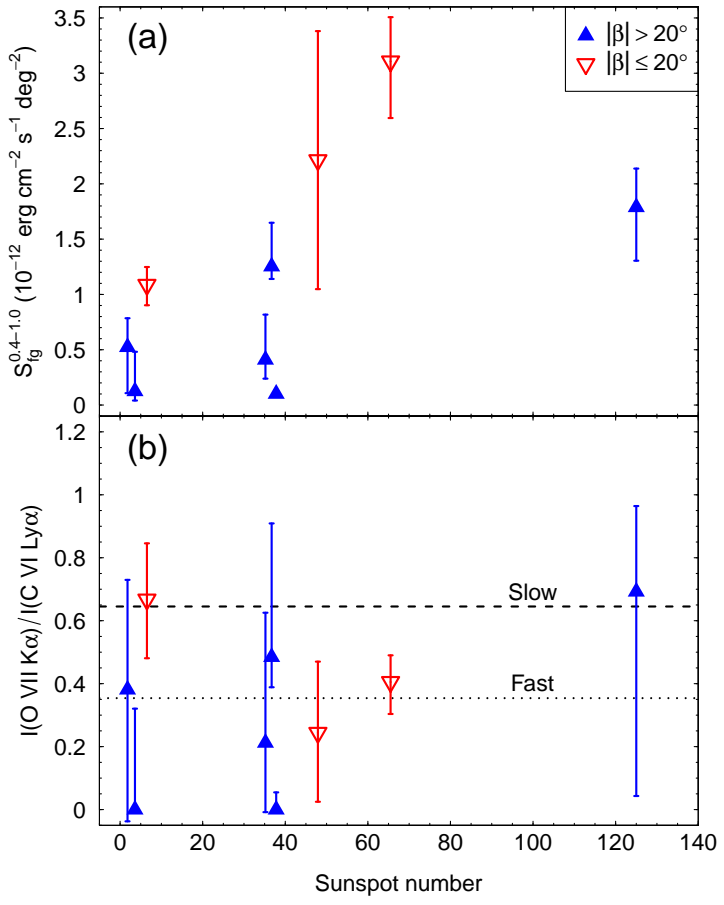

Figure 13. Foreground (a) $0.4-1.0 \mathrm{keV}$ surface brightness and (b) $\mathrm{O}$ VII $\mathrm{K} \alpha / \mathrm{C}$ VI Ly $\alpha$ intensity ratio plotted against sunspot number. Foreground results are for the C14-SWCX model with Aspl09 abundances. Results for high and low ecliptic latitudes are shown with solid blue and open red triangles, respectively. The dotted and dashed lines in panel (b) show the expected intensity ratios for the fast and slow solar wind, respectively (see text for details).

a foreground surface brightness of only $\left(0.41_{-0.17}^{+0.41}\right) \times$ $10^{-12} \mathrm{erg} \mathrm{cm}^{-2} \mathrm{~s}^{-1} \mathrm{deg}^{-2}$ (Henley et al. 2015a), similar to the values seen on high-ecliptic-latitude sight lines near solar minimum, despite being taken in 2013 February, only $\sim 9$ months before solar maximum. However, as noted by Henley et al. (2015a), the sun was less active during this maximum than during preceding maxima (based on the sunspot number and the solar 1-8 $\AA$ flux; Winter \& Balasubramaniam 2014), which may have affected the heliospheric SWCX emission.

Figure 13(b) shows the ratio of the foreground O VII $\mathrm{K} \alpha$ and $\mathrm{C}$ VI Ly $\alpha$ intensities, $I(\mathrm{O}$ VII K $\alpha) / I(\mathrm{C}$ VI Ly $\alpha)$, against sunspot number. We concentrate on this ratio because, in general, we do not detect foreground O VIII emission with this model, meaning that we can only place upper limits on the O VIII/O VII ratio. However, it should be noted that C VI Ly $\alpha$ lies near the lower limit of the XMM-Newton and Suzaku bandpasses (in fact, for some fits, C VI Ly $\alpha$ lies below the energy band used, and is constrained indirectly via the C14-SWCX line ratios). As the calibration is uncertain at these low energies, it is possible that the uncertainties in Figure 13(b) are underestimated.

Figure 13(b) also shows the intensity ratios expected for the fast and slow solar winds, given by

$$
\frac{I(\mathrm{O} \text { VII K } \alpha)}{I(\mathrm{C} \text { VI Ly } \alpha)}=\frac{n\left(\mathrm{O}^{7+}\right) \sigma\left(\mathrm{O}^{7+}+\mathrm{H}\right) y(\mathrm{O} \text { VII K } \alpha)}{n\left(\mathrm{C}^{6+}\right) \sigma\left(\mathrm{C}^{6+}+\mathrm{H}\right) y(\mathrm{C} \text { VI Ly } \alpha)},
$$

where $n(X)$ is the density of ion $X$ in the solar wind, $\sigma(X+\mathrm{H})$ is the CX cross-section for $X$ reacting with 
hydrogen, ${ }^{18}$ and $y(L)$ is the yield for line $L$. To calculate the expected intensity ratios, we use ion abundances from Schwadron \& Cravens (2000, Table $1 ; n\left(\mathrm{O}^{7+}\right) / n\left(\mathrm{C}^{6+}\right)=$ 0.353 and 0.629 for the fast and slow solar winds, respectively) and cross-sections from Koutroumpa et al. (2006, Table 1; $\sigma\left(\mathrm{O}^{7+}+\mathrm{H}\right) / \sigma\left(\mathrm{C}^{6+}+\mathrm{H}\right)=0.817$ and 0.799 for the fast and slow solar winds, respectively). For the line yields, we use the CX line ratio data from Cumbee et al. (2014), assuming that the emission from higher-order lines not included in the model is negligible: $y(\mathrm{O}$ VII $\mathrm{K} \alpha)=0.902$ and $y(\mathrm{C}$ VI Ly $\alpha)=0.719 .{ }^{19}$ Thus, we obtain $I(\mathrm{O}$ VII $\mathrm{K} \alpha) / I(\mathrm{C}$ VI Ly $\alpha)=0.354$ and 0.645 for the fast and slow solar winds, respectively.

Some of the observed ratios are as expected. For example, the observation with the highest sunspot number, which was taken near solar maximum, and the lowecliptic-latitude observation that was taken near solar minimum are both expected to probe the slow solar wind - both yield O VII/C VI emission ratios consistent with the slow solar wind value. On the other hand, some observed emission ratios are not as expected. For example, at intermediate sunspot numbers, two observations are toward low ecliptic latitudes, and are thus expected to probe the slow solar wind. However, the O VII/C VI emission ratios for these two observations are less than that expected for the slow solar wind. While these discrepancies may simply be due to the calibration uncertainty near the C VI lines, we note the possibility that these discrepancies are due to localized variations in the solar wind (such as coronal mass ejections; CMEs), crossing the line of sight, or to variations in the geocoronal SWCX emission.

In summary, only some aspects of our set of foreground emission measurements are as expected from a model of heliospheric SWCX emission from a smooth solar wind Koutroumpa et al. 2006). Deviations from these expectations may be due to localized solar wind variations, such as CMEs, crossing the line of sight (Smith et al. 2005; Koutroumpa et al. 2007; Henley \& Shelton 2008; Carter et al. 2010), or due to enhancements in the geocoronal emission (Wargelin et al. 2004; Snowden et al. 2004; Fuiimoto et al. 2007; Snowden et al. 2009; Ezoe et al. 2010; Wargelin et al. 2014). The details of the latitudinal variation of the solar wind may also be important in explaining the discrepancies between the Koutroumpa et al. (2006) model and the observations. Our measurements of the foreground SWCX emission will provide useful constraints on future SWCX models.

\subsection{Limits on the Local Bubble Emission}

Koutroumpa et al. (2007) developed the general Koutroumpa et al. (2006) heliospheric SWCX model (discussed above) to take into account contemporaneous

\footnotetext{
18 We are ignoring CX interactions with helium. However, as helium is an order of magnitude less abundant than hydrogen (except within one or two AU of the Sun) and as CX cross-sections involving helium are typically smaller than those involving hydrogen (e.g., Koutroumpa et al. 2006, Table 1), ignoring helium should not greatly affect our results. Note that the C14-SWCX model is based on line ratios for ions interacting with hydrogen only.

19 These line ratios were calculated for a collision speed of $\sim 400 \mathrm{~km} \mathrm{~s}^{-1}$, appropriate for the slow solar wind (Smith et al. 2003). Relevant data for a collision speed appropriate for the fast solar wind $\left(\sim 700-800 \mathrm{~km} \mathrm{~s}^{-1}\right)$ are not available.
}

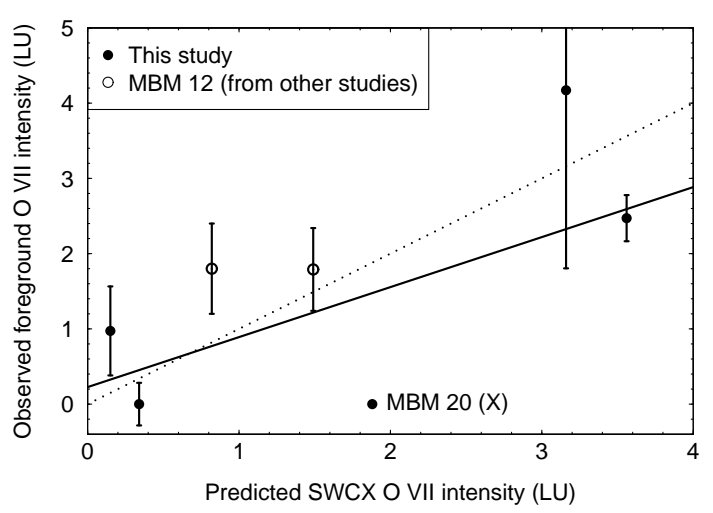

Figure 14. Comparison of observed foreground O VII intensity with heliospheric SWCX model predictions (Koutroumpa et al. 2011, Table 5). Solid symbols indicate our measurements. For the purposes of this plot we show $1 \sigma$ errors (see text for details). Open symbols represent measurements from Chandra and XMM-Newton observations of MBM 12, not included in our sample (Smith et al. 2005; (Koutroumpa et al.|2011). The dotted line indicates equality. The solid line is the best-fit linear regression line (excluding the $X M M$-Newton observation of MBM 20, which is labeled; see text for details).

solar wind conditions during a given observation, and to include (if necessary) the emission from localized solar wind enhancements crossing the line of sight. Here, we follow Koutroumpa et al. (2007, 2011) and use such predictions to place limits on the emission attributable to the LB.

Heliospheric SWCX predictions for a subset of our shadowing observations (specifically, the observations of MBM 12, MBM 20, and the filament) are summarized in Koutroumpa et al. (2011. Table 5). Figure 14 compares the predicted O VII intensities with our measurements. The figure also includes two additional measurements from Chandra and XMM-Newton observations of MBM 12, not included in our sample (Smith et al. 2005; Koutroumpa et al. 2011). In general, the predictions are in good agreement with the observations. The one exception is the XMM-Newton observation of MBM 20 (labeled in the plot), for which the model significantly overpredicts the foreground O VII intensity.

Following Koutroumpa et al. (2007, 2011), we will use the data in Figure 14 to place limits on the LB O VII intensity. In this analysis, we are assuming that the plasma filling the LB is uniform. Furthermore, we are assuming that the extent of the LB toward the three clouds whose results are included in Figure 14 is similar. From the maps of the Local Cavity in Lallement et al. (2003), we estimate that the extent of the Local Cavity (which we assume to be the same as that of the LB) is $\sim 100-150 \mathrm{pc}$ toward these three clouds. This analysis involves fitting a straight line to the data in Figure 14 (Koutroumpa et al. 2007, 2011). Since weighted-least-squares linear regression requires symmetrical errors, for each data point we used the larger of the upper and lower errors, rescaled from a $90 \%$ confidence interval to a $1 \sigma$ confidence interval (these are the errors shown in Figure 14). We excluded the XMM-Newton observation of MBM 20, as it is such an extreme outlier.

The best fit straight line (solid line in Figure 14) has a gradient of $0.66 \pm 0.18$ and an intercept of $0.23 \pm 0.38$ L.U. ( $1 \sigma$ errors). The gradient is consistent with that obtained by Koutroumpa et al. (2011), using the then- 
available measurements of the foreground O VII intensity $(0.65 \pm 0.16)$. However, our intercept is significantly less than theirs $(1.07 \pm 0.47$ L.U.). This is because some of the foreground O VII intensities used in our fit (obtained using the C14-SWCX model with Aspl09 abundances) are lower than those used by Koutroumpa et al. (2011). If we repeat our analysis using the foreground O VII intensities obtained using AG89 abundances, we obtain an intercept of $1.30 \pm 0.31$ L.U., consistent with Koutroumpa et al.'s value. However, as noted in Section 4.2, results obtained using AG89 abundances may be unreliable.

Koutroumpa et al. (2011) suggested that their nonzero intercept was evidence of O VII emission from the hot LB. However, our intercept is consistent with zero. Rescaling the $1 \sigma$ confidence interval on the intercept to a $90 \%$ confidence interval, we find that the LB contributes $\lesssim 0.8$ L.U. to the foreground O VII emission (for an LB path length of $\sim 100 \mathrm{pc}$ ).

\subsection{Halo Emission}

\subsubsection{Comparison with Previous Halo Studies}

We begin our discussion of the halo X-ray emission by comparing our measurements with those from other recent studies of the halo with CCD-resolution spectra - see Figure 7. The black crossed diamonds indicate the quartiles from an XMM-Newton survey of the halo emission (encompassing 110 high-Galactic-latitude $\left(|b|>30^{\circ}\right)$ sight lines; HS13). HS13 assumed an LBlike foreground with $T_{\mathrm{fg}}=1.2 \times 10^{6} \mathrm{~K}$. For each sight line the foreground emission measure (typically $\left.\sim(3-6) \times 10^{-3} \mathrm{~cm}^{-6} \mathrm{pc}\right)$ was fixed using nearby shadowing measurements of the foreground $1 / 4 \mathrm{keV}$ count rate (Snowden et al. 2000). Thus, HS13's foreground modeling method involved extrapolating results from the $1 / 4 \mathrm{keV}$ band to the higher-energy XMM-Newton band, which may have introduced a bias to their halo measurements. The black stars show results from a study of the halo emission using 12 high-latitude $\left(|b|>20^{\circ}\right)$ Suzaku observations (Yosh09). Yosh09 used the same LB-like foreground model for all their sight lines, with $T_{\text {fg }} \sim 1.3 \times 10^{6} \mathrm{~K}$ and $\mathcal{E}_{\mathrm{fg}} \sim 7 \times 10^{-3} \mathrm{~cm}^{-6} \mathrm{pc}^{20}$ Note that, because Yosh09 and HS13 assumed AG89 abundances, we have rescaled their halo emission measures in Figure 7 to account for the different oxygen abundances in AG89 and Aspl09 (see footnote 11).

The halo parameters that we obtain using our reference model are generally within the ranges of values reported by HS13. When we compare our values with their lower and upper quartiles, we find that some of our temperatures are on the low side compared to their values. This difference is not due to HS13 using the Raymond \& Smith (1977 and updates) code, whereas

\footnotetext{
20 Note that HS13's and Yosh09's foreground emission measures are somewhat smaller than those we measured using the LB model with Aspl09 abundances (Table 2 Section (c)). This is partly because they assumed AG89 abundances, and partly because their assumed foreground temperatures are higher than some of our best-fit LB temperatures (both of which result in more emission in the XMM-Newton/Suzaku band per unit emission measure). HS13 and Yosh09's foreground models have 0.4$1.0 \mathrm{keV}$ surface brightnesses of $\sim(0.6-1.1) \times 10^{-12}$ (typically) and $\sim 1.8 \times 10^{-12} \mathrm{erg} \mathrm{cm}^{-2} \mathrm{~s}^{-1} \mathrm{deg}^{-2}$, respectively, placing them well within the range of foreground surface brightnesses that we measured (see, e.g., Figure 6).
}

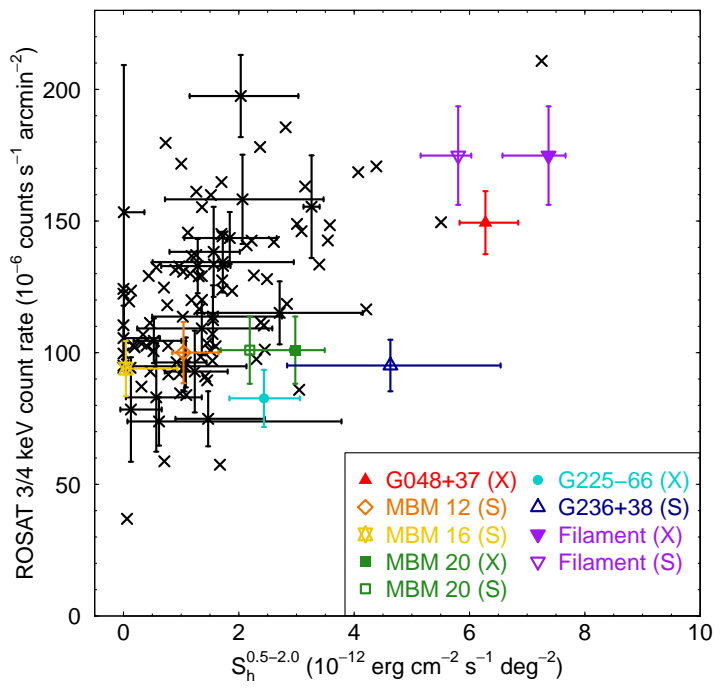

Figure 15. Comparison of halo surface brightnesses (abscissae) from our shadowing measurements (colored symbols matching those used in Figure 7 see key) and from HS13 (black diagonal crosses) with $3 / 4 \mathrm{keV}$ (R45) count rates for the same directions from the ROSAT All-Sky Survey (ordinates; Snowden et al. 1997). Note that, to avoid clutter, we only show error bars for a sample of the data points from HS13.

we used APEC-for temperatures around $2 \times 10^{6} \mathrm{~K}$, a given observed halo O VIII/O VII ratio implies a temperature $\sim 10^{5} \mathrm{~K}$ lower with the Raymond \& Smith code than with APEC. Nor is this difference due to HS13 using AG89 abundances, which, in our analysis, resulted in higher halo temperatures than the Aspl09 abundances (Figure 9(b)) - the foreground model was fixed during HS13's fitting, and so the discussion in Section 4.2 is not applicable to their results. However, it should be noted that the median halo temperature from the shadowing measurements, $2.06 \times 10^{6} \mathrm{~K}$, lies between HS13's lower quartile and median, suggesting that any difference between the two sets of results is not significant.

In contrast, the median halo surface brightness from the shadowing measurements, $2.98 \times$ $10^{-12} \mathrm{erg} \mathrm{cm}^{-2} \mathrm{~s}^{-1} \mathrm{deg}^{-2}$, lies above the upper quartile from HS13, suggesting that the shadowing measurements tend to yield systematically brighter halo emission than HS13's measurements. However, the halo surface brightness is known to vary across the sky (Yosh09; HS13), and so it is possible that the shadowing measurements happen to sample brighter-than-typical regions of the halo. To check this, we compared the halo surface brightnesses from our measurements and from HS13 with the $3 / 4 \mathrm{keV}$ count rates for the same directions from the ROSATAllSky Survey (Snowden et al. 1997). The ROSAT rates were extracted from circular regions of radius 0.5 , centered on each observation's pointing direction (for the shadowing observations, we used the off-shadow pointing). The comparison is shown in Figure 15. Note that, for most sight lines shown in the figure, the column density is in the range $\sim(1-3) \times 10^{20} \mathrm{~cm}^{-2}$, over which range the observed $3 / 4 \mathrm{keV}$ count rate of a $\sim 2 \times 10^{6} \mathrm{~K}$ halo plasma will decrease by $\lesssim 20 \%$. Hence, the total $3 / 4 \mathrm{keV}$ count rate (plotted in Figure 15) should be strongly correlated with the halo's intrinsic $3 / 4 \mathrm{keV}$ count rate.

For lower $3 / 4 \mathrm{keV}$ count rates $(\lesssim 110 \times$ 
$10^{-6}$ counts $\mathrm{s}^{-1} \operatorname{arcmin}^{-2}$ ), the surface brightness measurements from our shadowing measurements are similar to the values obtained by HS13. (The best-fit surface brightness for G236+38 (blue triangle in Figure 15) is somewhat outlying, but the uncertainty on this value is relatively large.) Specifically, for sight lines with $3 / 4 \mathrm{keV}$ count rates of $(80-105) \times 10^{-6}$ counts $\mathrm{s}^{-1} \operatorname{arcmin}^{-2}$, the median \pm MADN $^{21}$ surface brightnesses are $(2.31 \pm 1.44) \times 10^{12}$ and $(0.95 \pm 0.75) \times 10^{-12} \mathrm{erg} \mathrm{cm}^{-2} \mathrm{~s}^{-1} \mathrm{deg}^{-2}$ from our shadowing measurements and from HS13, respectively. A Mann-Whitney $U$ test (e.g., Barlow 1989; Wall \& Jenkins 2003) indicates that the difference in the median surface brightnesses is not statistically significant at the $1 \%$ level $(U=49$, two-sided $p$ value $=0.063)$.

At higher $3 / 4 \mathrm{keV}$ count rates $(\geq 145 \times$ $10^{-6}$ counts $\mathrm{s}^{-1} \operatorname{arcmin}^{-2}$ ), the surface brightnesses from our shadowing measurements are larger than nearly all of the corresponding values from HS13. The median \pm MADN surface brightnesses are $(6.28 \pm 0.70) \times 10^{12}$ and $(2.22 \pm 1.40) \times 10^{-12} \mathrm{erg} \mathrm{cm}^{-2} \mathrm{~s}^{-1} \mathrm{deg}^{-2}$, respectively. However, a Mann-Whitney $U$ test indicates that this difference is also not statistically significant at the $1 \%$ level $(U=2$, two-sided $p$ value $=0.011)$. Furthermore, two of the three shadowing measurements in this regime are of the filament (purple triangles in Figure 151 - this direction may not be representative of the halo, as an extraplanar supernova remnant may lie in this direction (Lei et al. 2009). Therefore, with the possible exception of the very brightest directions, there appears to be no significant systematic difference between the halo surface brightnesses obtained from our shadowing observations and those obtained by HS13.

When we compare our results with those from Yosh09, we find that the emission measures (after accounting for the different assumed oxygen abundances) and surface brightnesses are in good agreement, whereas our halo temperatures tend to be lower than Yosh09's (see Figure [7). The median \pm MADN temperatures from our and Yosh09's measurements are $(2.06 \pm 0.22) \times 10^{6}$ and $(2.47 \pm 0.41) \times 10^{6} \mathrm{~K}$, respectively. ${ }^{22}$ A Mann-Whitney $U$ test indicates that this difference is statistically significant at the $1 \%$ level $(U=7$, two-sided $p$ value $=0.0026)$.

The discrepancy between the two set of results may be due to Yosh09 assuming the same foreground model for all their sight lines, whereas our results indicate that the foreground surface brightness can vary significantly from observation to observation (see Figure 6). If Yosh09's foreground model is too bright for a given observation, too much $\mathrm{O}$ VII will be attributed to the foreground; as a result, the halo $\mathrm{O}$ VII/O VIII ratio will be underestimated, and the halo temperature overestimated. Note also that, if the foreground model is too bright, the halo surface brightness will correspondingly be underestimated. Thus, if Yosh09's foreground model is too bright

21 Normalized median absolute deviation - for a set of numbers $\boldsymbol{x} \equiv\left\{x_{i}\right\}, \operatorname{MADN}(\boldsymbol{x})=1.4826 \times \operatorname{median}\left(\left|x_{i}-\operatorname{median}(\boldsymbol{x})\right|\right)$. The numerical factor ensures that the MADN of a normal distribution is equal to its standard deviation (e.g. Feigelson \& Babu 2012).

22 Each of our data sets includes a sight line on which the halo temperature was fixed (MBM 16 in our data set, LH-2 in Yosh09's). These sight lines were excluded from the calculation of the median temperatures.
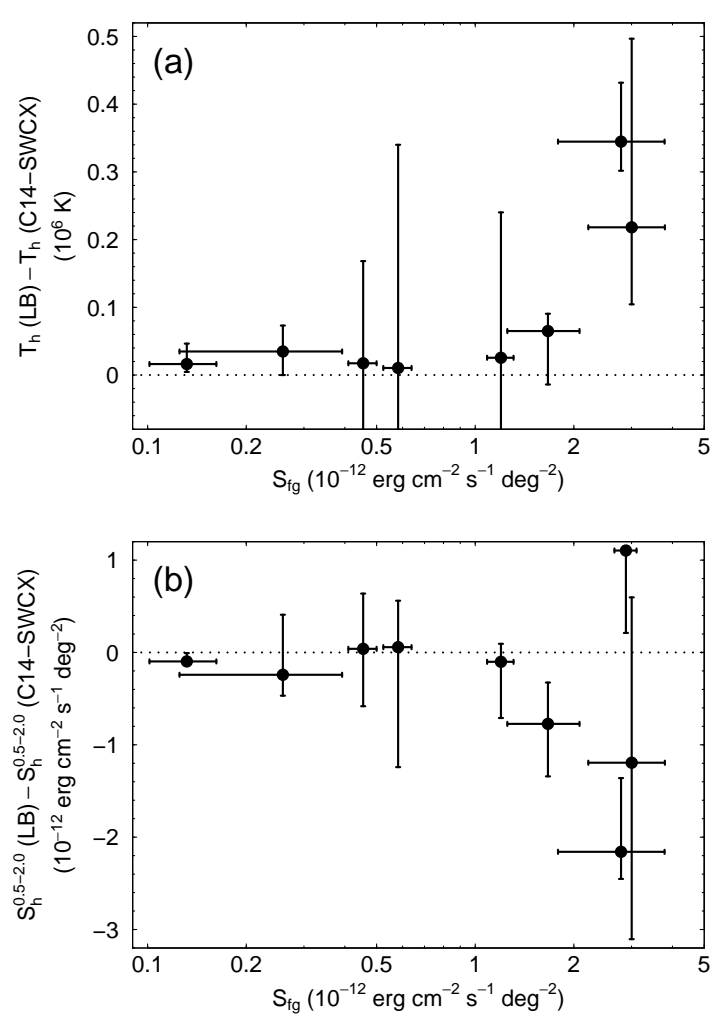

Figure 16. Differences between in the halo (a) temperatures and (b) $0.5-2.0 \mathrm{keV}$ surface brightnesses obtained with the LB and C14-SWCX foreground models, as functions of the $0.4-1.0 \mathrm{keV}$ foreground surface brightness. For each shadowing observation, the abscissa indicates the mean foreground surface brightness from the two foreground models, while the error bar indicates the lower and higher values. The ordinate shows the difference between the halo measurements obtained with the two models, while the error bar indicates the uncertainty on this difference due to the uncertainty on the measurement using the C14-SWCX model. Note that the plots include the results for G225-66 from Henley et al. (2015a). The temperature plot does not include MBM 16, as the temperature was held fixed for this observation (see Section [3).

for some of their observations, we would expect to see an anticorrelation between their halo temperatures and surface brightnesses. In the lower panel of Figure 7, we can see that the Yosh09 sight lines with higher halo temperatures tend to have lower surface brightnesses. Although this anticorrelation is not statistically significant at the $1 \%$ level (Kendall's $\tau$ (e.g., Press et al. 1992) is -0.56 , with a $p$ value of 0.017 ), it does suggest that Yosh09 may have overestimated the halo temperature on a few of their sight lines.

\subsubsection{Uncertainties or Biases in the Halo Measurements}

The uncertainties quoted in our tables of results (Tables 2 and 3) are purely statistical uncertainties. In this section, we discuss possible uncertainties or biases in our halo measurements that may arise from the spectral modeling in addition to the statistical uncertainties.

We described the results obtained with the different foreground models in Section 4.1 and found that, in general, the C14-SWCX and LB foreground models yielded consistent halo results. However, we noted some exceptions to this general result. These occur when the foreground is relatively bright, as shown in Figure 16, which shows the difference between the halo (a) temperatures 
and (b) $0.5-2.0 \mathrm{keV}$ surface brightnesses obtained using the LB and C14-SWCX foreground models, as functions of the foreground surface brightness. These differences may be interpreted in two ways. They may be interpreted as the uncertainty in the halo parameters due to the fact that we do not know the relative contributions of SWCX and LB emission to the XMM-Newton/Suzaku foreground. Alternatively, if the LB emission is negligible in the XMM-Newton/Suzaku band, then these differences represent the bias in the halo parameters due to assuming an LB-like foreground model.

When the foreground $0.4-1.0 \mathrm{keV}$ surface brightness is $\lesssim 1.5 \times 10^{-12} \mathrm{erg} \mathrm{cm}^{-2} \mathrm{~s}^{-1} \mathrm{deg}^{-2}$, the differences in the halo results obtained with the two foreground models are negligible: $\lesssim 0.05 \times 10^{6} \mathrm{~K}$ and $\lesssim 0.2 \times$ $10^{-12} \mathrm{erg} \mathrm{cm}^{-2} \mathrm{~s}^{-1} \mathrm{deg}^{-2}$ on the halo temperature and surface brightness, respectively. For foreground surface brightnesses of $\sim 3 \times 10^{-12} \mathrm{erg} \mathrm{cm}^{-2} \mathrm{~s}^{-1} \mathrm{deg}^{-2}$, however, these differences are $\sim(0.2-0.3) \times 10^{6} \mathrm{~K}$ and $\sim(1-2) \times 10^{-12} \mathrm{erg} \mathrm{cm}^{-2} \mathrm{~s}^{-1} \mathrm{deg}^{-2}$, respectively (the observations in question are G236+38, MBM 16, and the XMM-Newton observation of the filament). For G236+38, these differences are similar in size to the statistical uncertainties. For MBM 16, the difference in the halo surface brightnesses from the two foreground models is of the opposite sign to the other observations' differences (note, however, that halo emission was not detected using the C14-SWCX for this observation, so a negative surface brightness difference is not possible).

These results mean that the uncertainty in the true nature of the foreground X-ray emission has a negligible effect on the halo measurements if the 0.4 $1.0 \mathrm{keV}$ foreground surface brightness is $\lesssim 1.5 \times$ $10^{-12} \mathrm{erg} \mathrm{cm}^{-2} \mathrm{~s}^{-1} \mathrm{deg}^{-2}$. If the foreground is brighter than this, then assuming an LB-like foreground model when the true XMM-Newton/Suzaku foreground is dominated by SWCX may bias the halo temperature upward by $\sim(0.2-0.3) \times 10^{6} \mathrm{~K}$ and the halo surface brightness downward by $\sim(1-2) \times 10^{-12} \mathrm{erg} \mathrm{cm}^{-2} \mathrm{~s}^{-1} \mathrm{deg}^{-2}$, if the halo temperature is a free parameter. Alternatively, if the true foreground in the XMM-Newton/Suzaku band is a mixture of LB and SWCX emission, then the uncertainty in this mixture results in an uncertainty of up to $\sim(0.2-0.3) \times 10^{6} \mathrm{~K}$ and $\sim(1-2) \times$ $10^{-12} \mathrm{erg} \mathrm{cm}^{-2} \mathrm{~s}^{-1} \mathrm{deg}^{-2}$ in the temperature and surface brightness of the halo, respectively. Uncertainties in the CX line ratios, which we are unable to quantify, could also contribute to the uncertainty in the halo emission, if the foreground is relatively bright.

Our sample includes two shadows, MBM 20 and the filament, that have been observed twice, once each with XMM-Newton and Suzaku. For each shadow, the two observatories yield halo temperatures that differ by $\sim 0.2 \times$ $10^{6} \mathrm{~K}$ (using our reference model). However, there is not a systematic difference between the two observatories$X M M$-Newton yields a lower temperature than Suzaku for MBM 20, but a higher temperature for the filament. XMM-Newton yields higher halo surface brightnesses than Suzaku for both shadows, by $0.8 \times 10^{-12}$ and $1.6 \times 10^{-12} \mathrm{erg} \mathrm{cm}^{-2} \mathrm{~s}^{-1} \mathrm{deg}^{-2}$ for MBM 20 and the filament, respectively. Note that these differences are not statistically significant for MBM 20, given the rela- tively large uncertainties on the Suzaku measurements, but are statistically significant for the filament. The differences between the results from the two satellites are unlikely to be due to our assumed column densities, since, for each cloud, we used similar on- and off-shadow column densities for the XMM-Newton and Suzaku observations. Instead, the differences may be due to our assuming that, for a given shadowing observation, the foreground is identical in the on- and off-shadow spectra. If the SWCX emission changes between the two pointings, the resulting halo measurement would be inaccurate. However, if this is the sole explanation for the differences between the XMM-Newton and Suzaku measurements, we might expect the MBM 20 measurements to exhibit greater differences than the filament measurements, as the on- and off-shadow MBM 20 pointings were further apart in time (for both the XMM-Newton and the Suzaku observations). Other possible explanations include halo structure on scales smaller than the angular separation between the on- and off-shadow pointings (our spectral analysis also assumes that the halo emission is identical in the two spectra), or inaccurate modeling of the soft proton contamination in the XMM-Newton spectra. Regardless of the source of the differences between the XMM-Newton and Suzaku measurements of the halo emission in the directions of MBM 20 and the filament, the existence of these differences implies that there may be uncertainties of up to $\sim 0.2 \times 10^{6} \mathrm{~K}$ and $\sim 25 \%$ in the temperature and surface brightness of the halo, respectively, in addition to the statistical uncertainties.

\subsubsection{Testing Models of the Galactic Halo Emission}

We modeled the Galactic halo emission in the XMMNewton/Suzaku band using a $1 T$ thermal plasma model, assuming CIE. It should be noted that such a model is a characterization of the emission, and does not necessarily reflect the true thermodynamic state of the halo gas. Our median halo temperature and emission measure (obtained using our reference model) predict a halo $1 / 4 \mathrm{keV}$ ROSAT count rate of $\sim 400 \times 10^{-6}$ counts s ${ }^{-1}$ arcmin $^{-2}$, $\sim 1 / 3$ of the median halo count rate detected by Snowden et al. (2000) in their study of X-ray shadows in the ROSAT All-Sky Survey. This discrepancy indicates the need for additional halo emission components. Kuntz \& Snowden (2000) also showed that a $1 T$ model cannot adequately model the halo emission down to $\sim 0.1 \mathrm{keV}$, and used a two-temperature halo model in their analysis of the ROSAT All-Sky Survey. Other studies have used X-ray and far-ultraviolet emission data to consider the distribution of hot halo gas down to $\sim 1 \times 10^{5} \mathrm{~K}$, using differential emission measure models (Shelton et al. 2007; Lei et al. 2009). Recombination emission from overionized halo plasma may be important (e.g., Breitschwerdt \& Schmutzler 1994; de Avillez \& Breitschwerdt 2012; although see discussion in Henley et al. 2015b). In addition, Henley et al. (2015b) recently discussed the possibility that CX emission, rather than thermal emission from hot gas, may contribute significantly to the observed halo emission.

Despite the uncertainties in the true nature of the Galactic halo emission, and of the hot halo gas, measurements of the Galactic halo emission that use a $1 T$ halo model can still be used to test physical models of the halo emission, even if the emission is from multitem- 
perature gas, or is from gas out of ionization equilibrium, or is due to CX. As we have noted previously, the key is to ensure that the predicted spectra are characterized in the same way as the observed spectra-the X-ray temperatures, emission measures, and surface brightnesses that result from this characterization can be compared with the observed values (Henley et al. 2010, 2015b).

In Section 5.4.1, we showed that, with the possible exception of the very brightest directions, there appears to be no significant systematic difference between the halo temperatures and surface brightnesses obtained from our shadowing observations and those obtained by HS13. This further strengthens the conclusion of Henlev et al. (2015a) that the latter results can be used to test models of the halo X-ray emission (Henley et al. 2015b). However, in Section 5.4.2, we discussed possible uncertainties or biases in the halo emission in addition to the statistical uncertainties. When testing halo models, one should take these potential uncertainties in the halo emission into account, which may limit the ability of measurements of the halo X-ray emission to discriminate between models.

\section{SUMMARY AND CONCLUSIONS}

We have analyzed eight XMM-Newton and Suzaku observations of six shadowing clouds, including the first such observations from the northern Galactic hemisphere (Section 2). To our results we added recently published results from a seventh cloud (Henley et al. 2015a). Ours is the first uniform analysis of a sample of X-ray shadowing clouds with CCD-resolution spectra.

We tested a variety of foreground models - two that assume that the foreground emission is dominated by SWCX (C14-SWCX and ACX-SWCX) and one that assumes that it is from the hot LB (Section 3). We also tested three abundance tables-AG89 (commonly used in previous SXRB studies), Wilm00, and Aspl09. Our reference model was the C14-SWCX foreground model with Aspl09 abundances. We modeled the Galactic halo emission using a $1 T$ model - while this is a simplification of the true halo emission, such a model can still usefully characterize the halo emission (Section 5.4.3).

Our main results, and resulting conclusions, are as follows:

1. The measured foreground surface brightnesses span more than an order of magnitude, from $\sim 10^{-13}$ to $\sim 10^{-12} \mathrm{erg} \mathrm{cm}^{-2} \mathrm{~s}^{-1} \mathrm{deg}^{-2}(0.4-1.0 \mathrm{keV}$; Figure 6). The brighter foregrounds tend also to be harder. The halo temperatures are all around $2 \times$ $10^{6} \mathrm{~K}\left((1.6-2.3) \times 10^{6} \mathrm{~K}\right.$ for our reference model $)$, with detected emission measures and $0.5-2.0 \mathrm{keV}$ surface brightnesses of $(5-17) \times 10^{-3} \mathrm{~cm}^{-6} \mathrm{pc}$ and $(1-7) \times 10^{-12} \mathrm{erg} \mathrm{cm}^{-2} \mathrm{~s}^{-1} \mathrm{deg}^{-2}$, respectively (Figure 7). On one sight line (toward MBM 16) we do not detect halo emission with our reference model (a result previously reported by Ursino et al. 2014).

2. In general, the Wilm00 and Aspl09 abundances yield similar results, due to the similarity of the carbon, nitrogen, and oxygen abundances. However, the AG89 abundances tend to yield higher foreground surface brightnesses and higher halo temperatures. These differences are due to AG89's higher carbon, nitrogen, and oxygen abundances
(Section 4.2). Since these abundances are almost certainly too high for the interstellar medium, using AG89 abundances in shadowing analyses may yield inaccurate results.

3. The ACX-SWCX model tends to attribute less O VII emission to the foreground than the other models, which can affect the halo measurements (Section 4.1). This is due to the fact that this model tends to produce relatively more foreground emission above $\sim 0.6 \mathrm{keV}$ than the other foreground models; this in turn is likely an artifact of the assumption that the solar wind ion distribution can be described by a single temperature. Since this is not a good description of the true solar wind ion distribution, the results obtained with a singletemperature ACX-SWCX model may be unreliable (Section 5.1).

4. We compared our measurements of the foreground surface brightness and the foreground O VII/C VI intensity ratio with general predictions of heliospheric SWCX emission from a smooth solar wind (Koutroumpa et al. 2006). Only some aspects of our observations were as expected, indicating the importance of other processes (such as emission from localized solar wind enhancements, or enhancements in the geocoronal SWCX emission; Section [5.2).

5. By comparing our measurements of the foreground O VII intensity with observation-specific SWCX predictions (and excluding the XMMNewton MBM 20 measurement), we placed an upper limit of $\sim 0.8 \mathrm{~L} . \mathrm{U}$. (90\% confidence) on the LB O VII emission (for an LB path length of $~ 100 \mathrm{pc}$; Section [5.3).

6. Our halo measurements are generally in good agreement with those from recent non-shadowing studies of the halo (HS13; Yosh09). However, by assuming a uniform foreground model, Yosh09 may have overestimated the halo temperature on some of their sight lines (Section 5.4.1).

7. When the foreground is relatively faint $(\lesssim 1.5 \times$ $10^{-12} \mathrm{erg} \mathrm{cm}^{-2} \mathrm{~s}^{-1} \mathrm{deg}^{-2}$ in the $0.4-1.0 \mathrm{keV}$ band), the C14-SWCX and LB foreground models yield similar results for the halo. If the foreground is brighter than this, and is truly dominated by SWCX emission, then assuming an LBlike foreground model may bias the halo temperature upward and the halo surface brightness downward. Alternatively, if the foreground is in fact a mixture of SWCX and LB emission, the uncertainty in this mixture results in an uncertainty in the halo temperature and surface brightness (of up to $\sim(0.2-0.3) \times 10^{6} \mathrm{~K}$ and $\sim(1-2) \times$ $10^{-12}$ erg $\mathrm{cm}^{-2} \mathrm{~s}^{-1} \mathrm{deg}^{-2}$, respectively; Section 5.4.2). Differences in the halo results obtained for the same shadow with XMM-Newton and Suzaku suggest that there may be uncertainties in the halo temperature and surface brightness (of up to $\sim 0.2 \times 10^{6} \mathrm{~K}$ and $\sim 25 \%$, respectively), in addition to the statistical uncertainties (Section 5.4.2). Both types of uncertainty may limit 
the ability of X-ray measurements to discriminate between models of the Galactic halo emission.

We thank Phillip Stancil for detailed comments on the $l$ distributions resulting from CX. We also thank the anonymous referee, whose comments have helped improve this paper. This research is based on observations obtained with XMM-Newton, an ESA science mission with instruments and contributions directly funded by ESA Member States and NASA, and with Suzaku, a collaborative mission between JAXA and NASA. We acknowledge use of the $\mathrm{R}$ software package (R Development Core Team 2008). This research was funded by NASA grant NNX13AF69G, awarded through the Astrophysics Data Analysis Program.

\section{REFERENCES}

Anders, E., \& Grevesse, N. 1989, Geochim. Cosmochim. Acta, 53, 197 (AG89)

Arnaud, K. A. 1996, in ASP Conf. Ser. 101, Astronomical Data Analysis Software and Systems V, ed. G. H. Jacoby \& J. Barnes (San Francisco: ASP), 17

Asplund, M., Grevesse, N., Sauval, A. J., \& Scott, P. 2009, ARA\&A, 47, 481 (Aspl09)

Bałucińska-Church, M., \& McCammon, D. 1992, ApJ, 400, 699

Barlow, R. 1989, Statistics: A Guide to the Use of Statistical Methods in the Physical Sciences (Chichester: Wiley)

Brandt, W. N., \& Hasinger, G. 2005, ARA\&A, 43, 827

Breitschwerdt, D., \& Schmutzler, T. 1994, Nature, 371, 774

Burrows, D. N., \& Mendenhall, J. A. 1991, Nature, 351, 629

Carter, J. A., \& Sembay, S. 2008, A\&A, 489, 837

Carter, J. A., Sembay, S., \& Read, A. M. 2010, MNRAS, 402, 867

Chen, L.-W., Fabian, A. C., \& Gendreau, K. C. 1997, MNRAS, 285,449

Cravens, T. E. 2000, ApJL, 532, L153

Cumbee, R. S., Henley, D. B., Stancil, P. C., et al. 2014, ApJL, 787, L31

de Avillez, M. A., \& Breitschwerdt, D. 2012, ApJL, 756, L3

Egger, R. J., \& Aschenbach, B. 1995, A\&A, 294, L25

Ezoe, Y., Ebisawa, K., Yamasaki, N. Y., et al. 2010, PASJ, 62, 981

Feigelson, E. D., \& Babu, G. J. 2012, Modern Statistical Methods for Astronomy with R Applications (Cambridge: Cambridge University Press)

Foster, A. R., Ji, L., Smith, R. K., \& Brickhouse, N. S. 2012 ApJ, 756, 128

Fujimoto, R., Mitsuda, K., McCammon, D., et al. 2007, PASJ, 59, S133

Galeazzi, M., Gupta, A., Covey, K., \& Ursino, E. 2007, ApJ, 658, 1081

Galeazzi, M., Chiao, M., Collier, M. R., et al. 2014, Nature, 512, 171

Gupta, A., Galeazzi, M., Koutroumpa, D., Smith, R., \& Lallement, R. 2009, ApJ, 707, 644

Gupta, A., Mathur, S., Krongold, Y., Nicastro, F., \& Galeazzi, M. 2012, ApJL, 756, L8

Henley, D. B., \& Shelton, R. L. 2008, ApJ, 676, 335

Henley, D. B., \& Shelton, R. L. 2012, ApJS, 202, 14

Henley, D. B., \& Shelton, R. L. 2013, ApJ, 773, 92 (HS13)

Henley, D. B., Shelton, R. L., Cumbee, R. S., \& Stancil, P. C. 2015a, ApJ, 799, 117

Henley, D. B., Shelton, R. L., \& Kuntz, K. D. 2007, ApJ, 661, 304

Henley, D. B., Shelton, R. L., \& Kwak, K. 2014, ApJ, 791, 41

Henley, D. B., Shelton, R. L., Kwak, K., Hill, A. S., \& Mac Low, M.-M. 2015b, ApJ, 800, 102

Henley, D. B., Shelton, R. L., Kwak, K., Joung, M. R., \& Mac Low, M.-M. 2010, ApJ, 723, 935

Ishisaki, Y., Maeda, Y., Fujimoto, R., et al. 2007, PASJ, 59, S113

Jenkins, E. B. 2009, SSRv, 143, 205

Kalberla, P. M. W., Burton, W. B., Hartmann, D., et al. 2005, A\&A, 440, 775
Kortright, J. B., \& Thompson, A. C. 2009, in X-ray Data Booklet, ed. A. C. Thompson (Berkeley: Lawrence Berkeley National Laboratory), 1-8

Koutroumpa, D., Acero, F., Lallement, R., Ballet, J., \& Kharchenko, V. 2007, A\&A, 475, 901

Koutroumpa, D., Lallement, R., Kharchenko, V., \& Dalgarno, A. 2009, SSRv, 143, 217

Koutroumpa, D., Lallement, R., Kharchenko, V., et al. 2006, A\&A, 460, 289

Koutroumpa, D., Smith, R. K., Edgar, R. J., et al. 2011, ApJ, 726,91

Koyama, K., Tsunemi, H., Dotani, T., et al. 2007, PASJ, 59, S23

Kuntz, K. D., \& Snowden, S. L. 2000, ApJ, 543, 195

Kuntz, K. D., \& Snowden, S. L. 2008, A\&A, 478, 575

Kuntz, K. D., Snowden, S. L., \& Verter, F. 1997, ApJ, 484, 245

Lallement, R., Welsh, B. Y., Vergely, J. L., Crifo, F., \& Sfeir, D. 2003, A\&A, 411, 447

Lei, S., Shelton, R. L., \& Henley, D. B. 2009, ApJ, 699, 1891

McCammon, D., \& Sanders, W. T. 1990, ARA\&A, 28, 657

Miller, M. J., \& Bregman, J. N. 2013, ApJ, 770, 118

Nicastro, F., Zezas, A., Drake, J., et al. 2002, ApJ, 573, 157

Press, W. H., Teukolsky, S. A., Vetterling, W. T., \& Flannery, B. P. 1992, Numerical Recipes in C - The Art of Scientific Computing, 2nd edn. (Cambridge: Cambridge University Press)

R Development Core Team. 2008, R: A Language and Environment for Statistical Computing (Vienna, Austria: R Foundation for Statistical Computing)

Rasmussen, A., Kahn, S. M., \& Paerels, F. 2003, in The IGM/Galaxy Connection. The Distribution of Baryons at $z=0$, ed. J. L. Rosenberg \& M. E. Putman (Dordrecht: Kluwer), 109

Raymond, J. C., \& Smith, B. W. 1977, ApJS, 35, 419

Roberts, T. P., \& Warwick, R. S. 2001, in ASP Conf. Ser. 234, X-ray Astronomy 2000, ed. R. Giacconi, S. Serio, \& L. Stella (San Francisco: ASP), 569

Robertson, I. P., \& Cravens, T. E. 2003a, JGR, 108 (A10), 8031

Robertson, I. P., \& Cravens, T. E. 2003b, GeoRL, 30(8), 1439

Sanders, W. T., Kraushaar, W. L., Nousek, J. A., \& Fried, P. M. 1977, ApJL, 217, L87

Schlegel, D. J., Finkbeiner, D. P., \& Davis, M. 1998, ApJ, 500, 525

Schwadron, N. A., \& Cravens, T. E. 2000, ApJ, 544, 558

Sekiya, N., Yamasaki, N. Y., Mitsuda, K., \& Takei, Y. 2014, PASJ, 66, L3

Shelton, R. L., Sallmen, S. M., \& Jenkins, E. B. 2007, ApJ, 659, 365

Smith, E. J., Marsden, R. G., Balogh, A., et al. 2003, Science, 302,1165

Smith, R., \& Foster, A. 2014, The AtomDB Charge Exchange Model (http://www.atomdb.org/CX/acx_manual.pdf)

Smith, R. K., Brickhouse, N. S., Liedahl, D. A., \& Raymond, J. C. 2001, ApJL, 556, L91

Smith, R. K., Edgar, R. J., Plucinsky, P. P., et al. 2005, ApJ, 623, 225

Smith, R. K., Foster, A. R., Edgar, R. J., \& Brickhouse, N. S. 2014, ApJ, 787, 77

Smith, R. K., Bautz, M. W., Edgar, R. J., et al. 2007, PASJ, 59, S141

Snowden, S. L., Collier, M. R., Cravens, T., et al. 2009, ApJ, 691, 372

Snowden, S. L., Collier, M. R., \& Kuntz, K. D. 2004, ApJ, 610, 1182

Snowden, S. L., Cox, D. P., McCammon, D., \& Sanders, W. T. 1990, ApJ, 354, 211

Snowden, S. L., Freyberg, M. J., Kuntz, K. D., \& Sanders, W. T. 2000, ApJS, 128, 171

Snowden, S. L., \& Kuntz, K. D. 2013, Cookbook for Analysis Procedures for XMM-Newton EPIC MOS Observations of Extended Objects and the Diffuse Background, version 5.8

Snowden, S. L., McCammon, D., Burrows, D. N., \& Mendenhall, J. A. 1994, ApJ, 424, 714

Snowden, S. L., McCammon, D., \& Verter, F. 1993, ApJL, 409, L21

Snowden, S. L., Mebold, U., Hirth, W., Herbstmeier, U., \& Schmitt, J. H. M. M. 1991, Science, 252, 1529

Snowden, S. L., Egger, R., Freyberg, M. J., et al. 1997, ApJ, 485, 125 
Strüder, L., Briel, U., Dennerl, K., et al. 2001, A\&A, 365, L18

Tawa, N., Hayashida, K., Nagai, M., et al. 2008, PASJ, 60, S11

Turner, M. J. L., Abbey, A., Arnaud, M., et al. 2001, A\&A, 365, L27

Ursino, E., Galeazzi, M., Liu, W., et al. 2014, Poster at X-ray View of Galaxy Ecosystems (Chandra Science Workshop), Boston, MA

von Steiger, R., Schwadron, N. A., Fisk, L. A., et al. 2000, JGR, 105,27217

Wall, J. V., \& Jenkins, C. R. 2003, Practical Statistics for Astronomers (Cambridge: Cambridge University Press)

Wang, Q. D., \& Yu, K. C. 1995, AJ, 109, 698

Wargelin, B. J., Kornbleuth, M., Martin, P. L., \& Juda, M. 2014, ApJ, 796, 28
Wargelin, B. J., Markevitch, M., Juda, M., et al. 2004, ApJ, 607, 596

Watson, M. G., Schröder, A. C., Fyfe, D., et al. 2009, A\&A, 493, 339

Welsh, B. Y., \& Shelton, R. L. 2009, Ap\&SS, 323, 1

Wilms, J., Allen, A., \& McCray, R. 2000, ApJ, 542, 914 (Wilm00)

Winter, L. M., \& Balasubramaniam, K. S. 2014, ApJL, 793, L45

Yan, M., Sadeghpour, H. R., \& Dalgarno, A. 1998, ApJ, 496, 1044 Yao, Y., \& Wang, Q. D. 2007, ApJ, 658, 1088

Yoshino, T., Mitsuda, K., Yamasaki, N. Y., et al. 2009, PASJ, 61, 805 (Yosh09) 
Table 2

Spectral Fit Results

\begin{tabular}{|c|c|c|c|c|c|c|c|c|c|c|c|c|c|c|c|c|c|}
\hline \multirow[b]{2}{*}{$\begin{array}{c}\text { Shadow }^{\mathrm{a}} \\
\text { (1) }\end{array}$} & \multicolumn{12}{|c|}{ Foreground } & \multicolumn{4}{|c|}{ Halo } & \multirow[b]{2}{*}{$\begin{array}{l}\chi^{2} / \text { dof } \\
(10)\end{array}$} \\
\hline & \multicolumn{2}{|r|}{$\begin{array}{c}T_{\mathrm{fg}} \\
\left(10^{6} \mathrm{~K}\right) \\
(2)\end{array}$} & \multicolumn{2}{|c|}{$\begin{array}{c}\text { Normalization }^{\text {D }} \\
\text { (3) }\end{array}$} & \multicolumn{2}{|r|}{$\begin{array}{l}I(\mathrm{C} v)^{\mathrm{C}, \mathrm{e}} \\
(\text { L.U. }) \\
(4)\end{array}$} & \multicolumn{2}{|c|}{$\begin{array}{l}I(\mathrm{C} \mathrm{VI})^{\mathrm{d}, \mathrm{e}} \\
(\mathrm{L} . \mathrm{U} .) \\
(5)\end{array}$} & \multicolumn{2}{|c|}{$\begin{array}{l}I(\mathrm{O} \text { VII })^{\mathrm{c}} \\
(\mathrm{L} . \mathrm{U} .) \\
(6)\end{array}$} & \multicolumn{2}{|c|}{$\begin{array}{l}I(\mathrm{O} \text { VIII })^{\mathrm{d}} \\
(\mathrm{L} . \mathrm{U} .) \\
(7)\end{array}$} & \multicolumn{2}{|r|}{$\begin{array}{c}T_{\mathrm{h}} \\
\left(10^{6} \mathrm{~K}\right) \\
(8)\end{array}$} & \multicolumn{2}{|c|}{$\begin{array}{c}\mathcal{E}_{\mathrm{h}} \\
\left(10^{-3} \mathrm{~cm}^{-6} \mathrm{pc}\right) \\
(9)\end{array}$} & \\
\hline \multicolumn{18}{|c|}{ (a) C14-SWCX foreground model, Asplund et al. (2009) abundances } \\
\hline $\begin{array}{l}\text { G048+37 (X) } \\
\text { MBM } 12(\mathrm{~S})\end{array}$ & & $\cdots$ & & $\cdots$ & 0.00 & $(0.00,4.97)$ & $\begin{array}{l}5.19 \\
3.70\end{array}$ & $(4.45,6.01)$ & 2.52 & $(2.21,4.69)$ & 0.00 & $(0.00,0.25)$ & 2.10 & $(2.08,2.18)$ & 15.30 & $(14.21,16.69)$ & $2010.05 / 1822$ \\
\hline $\begin{array}{l}\text { MBM } 12(\mathrm{~S}) \\
\text { MBM } 16(\mathrm{~S})\end{array}$ & & $\begin{array}{l}\cdots \\
\cdots\end{array}$ & & $\begin{array}{l}\cdots \\
\cdots\end{array}$ & 12.59 & $(3.97,21.43)$ & $\begin{array}{r}3.70 \\
13.05\end{array}$ & $\begin{array}{c}(2.90,4.41) \\
(10.99 .15 .05)\end{array}$ & $\begin{array}{l}2.47 \\
5.29\end{array}$ & $\begin{array}{l}(1.97,2.86) \\
(4.24,6.01)\end{array}$ & $\begin{array}{l}0.00 \\
0.75\end{array}$ & $\begin{array}{l}(0.00,0.07) \\
(0.31 .1 .06)\end{array}$ & 1.61 & $\begin{array}{l}(1.40,1.83) \\
2.10^{\mathrm{f}}\end{array}$ & $\begin{array}{l}4.57 \\
0.10\end{array}$ & $\begin{array}{l}(3.71,7.24) \\
(0.00,2.28)\end{array}$ & $\begin{array}{l}410.71 / 358 \\
263.54 / 267\end{array}$ \\
\hline MBM $20(\mathrm{X})$ & & $\ldots$ & & $\ldots$ & 3.83 & $(1.31,5.37)$ & 1.10 & $(0.73,1.30)$ & 0.00 & $(0.00,0.06)$ & 0.00 & $(0.00,0.04)$ & 1.84 & $(1.81,1.86)$ & 9.36 & $(9.07,9.49)$ & $3973.74 / 3601$ \\
\hline MBM 20 (S) & & $\ldots$ & & $\ldots$ & 37.82 & $(21.56,53.94)$ & 2.55 & $(1.39,3.71)$ & 0.97 & $(0.00,1.74)$ & 0.00 & $(0.00,0.11)$ & 2.06 & $(1.73,2.20)$ & 5.51 & $(4.24,8.78)$ & $402.75 / 361$ \\
\hline $\mathrm{G} 236+38(\mathrm{~S})$ & & $\ldots$ & & $\ldots$ & 78.65 & $(0.00,156.63)$ & 13.64 & $(9.46,17.88)$ & 3.33 & $(0.52,6.24)$ & 0.00 & $(0.00,0.85)$ & 1.72 & $(1.44,1.83)$ & 17.11 & $(10.49,24.17)$ & $263.35 / 230$ \\
\hline Filament (X) & & $\ldots$ & & $\ldots$ & 0.00 & $(0.00,20.51)$ & 6.03 & $(4.43,6.58)$ & 4.17 & $(0.28,5.38)$ & 0.00 & $(0.00,0.20)$ & 2.32 & $(2.23,2.36)$ & 15.51 & (13.83,16.13) & $736.50 / 673$ \\
\hline Filament (S) & & $\cdots$ & & $\cdots$ & 9.21 & $(1.21,18.13)$ & 1.45 & $(0.59,2.18)$ & 0.00 & $(0.00,0.47)$ & 0.00 & $(0.00,0.05)$ & 2.14 & $(2.10,2.18)$ & 13.76 & $(12.22,14.30)$ & $550.18 / 398$ \\
\hline \multicolumn{18}{|c|}{ (b) ACX-SWCX foreground model, Asplund et al. (2009) abundances } \\
\hline $\mathrm{G} 048+37(\mathrm{X})$ & 2.22 & $(2.13,2.43)$ & 1.53 & $(1.28,2.03)$ & & $\ldots$ & & $\ldots$ & & $\ldots$ & & $\ldots$ & 1.88 & $(1.67,1.95)$ & 15.06 & $(13.91,16.73)$ & $1997.19 / 1824$ \\
\hline MBM $12(\mathrm{~S})$ & 1.71 & $(1.61,1.80)$ & 1.69 & $(1.44,1.97)$ & & $\ldots$ & & $\ldots$ & & $\ldots$ & & $\ldots$ & 1.58 & $(1.39,1.65)$ & 5.82 & $(4.45,8.46)$ & $428.35 / 360$ \\
\hline MBM 16 (S) & 1.89 & $(1.78,1.96)$ & 3.42 & $(2.92,3.76)$ & & $\ldots$ & & $\ldots$ & & $\ldots$ & & $\ldots$ & & $2.10^{\mathrm{f}}$ & 0.00 & $(0.00,1.51)$ & $265.04 / 268$ \\
\hline MBM $20(\mathrm{X})$ & 0.98 & $(0.94,0.99)$ & 3.86 & $(3.18,4.29)$ & & $\ldots$ & & $\ldots$ & & $\ldots$ & & $\ldots$ & 1.90 & $(1.87,1.91)$ & 8.87 & $(8.62,9.36)$ & $3975.93 / 3603$ \\
\hline MBM 20 (S) & 0.81 & $(0.72,0.89)$ & 30.88 & $(16.22,57.93)$ & & $\ldots$ & & $\ldots$ & & $\ldots$ & & $\ldots$ & 1.89 & $(1.76,2.06)$ & 7.47 & $(6.09,8.92)$ & $400.11 / 363$ \\
\hline $\mathrm{G} 236+38(\mathrm{~S})$ & 0.83 & $(0.76,0.91)$ & 102.70 & $(53.62,176.50)$ & & $\ldots$ & & $\ldots$ & & $\ldots$ & & $\ldots$ & 1.68 & $(1.60,1.77)$ & 22.53 & $(19.12,25.94)$ & $261.95 / 232$ \\
\hline Filament (X) & 1.93 & $(1.14,2.46)$ & 1.73 & $(1.21,2.00)$ & & $\cdots$ & & $\ldots$ & & $\cdots$ & & $\ldots$ & 2.19 & $(2.09,2.24)$ & 17.39 & $(15.52,19.77)$ & $733.06 / 675$ \\
\hline Filament (S) & 0.88 & $(0.74,1.05)$ & 8.92 & $(2.83,21.42)$ & & $\cdots$ & & $\cdots$ & & $\cdots$ & & $\cdots$ & 2.15 & $(2.11,2.19)$ & 13.60 & $(12.93,14.27)$ & $547.94 / 400$ \\
\hline \multicolumn{18}{|c|}{ (c) LB foreground model, Asplund et al. (2009) abundances } \\
\hline G048+37 (X) & 1.34 & $(1.26,1.40)$ & 12.01 & $(10.39,14.18)$ & & $\ldots$ & & $\ldots$ & & $\ldots$ & & $\ldots$ & 2.17 & $(2.14,2.26)$ & 12.80 & $(11.41,13.44)$ & $1998.69 / 1824$ \\
\hline MBM 12 (S) & 1.23 & $(1.12,1.30)$ & 10.15 & $(8.23,13.42)$ & & $\ldots$ & & $\ldots$ & & $\cdots$ & & $\ldots$ & 1.64 & $(1.41,1.96)$ & 3.95 & $(2.22,6.09)$ & $395.81 / 360$ \\
\hline MBM 16 (S) & 1.03 & $(0.86,1.13)$ & 42.29 & $(28.07,96.21)$ & & $\ldots$ & & $\ldots$ & & $\ldots$ & & $\ldots$ & & $2.10^{\mathrm{f}}$ & 2.80 & $(1.18,4.42)$ & $275.42 / 268$ \\
\hline MBM $20(\mathrm{X})$ & 0.77 & $(0.73,0.80)$ & 13.08 & $(10.50,15.57)$ & & $\ldots$ & & $\ldots$ & & $\ldots$ & & $\ldots$ & 1.86 & $(1.83,1.88)$ & 8.87 & $(8.67,9.05)$ & 4011.09/3603 \\
\hline MBM 20 (S) & 0.66 & $(0.56,0.83)$ & 133.17 & $(38.30,362.44)$ & & $\ldots$ & & $\ldots$ & & $\ldots$ & & $\ldots$ & 2.07 & $(1.88,2.19)$ & 5.61 & $(4.75,6.47)$ & $388.11 / 363$ \\
\hline $\mathrm{G} 236+38(\mathrm{~S})$ & 1.01 & $(0.64,1.10)$ & 66.87 & $(51.92,758.26)$ & & $\ldots$ & & $\ldots$ & & $\cdots$ & & $\ldots$ & 1.94 & $(1.69,2.16)$ & 9.64 & $(5.51,18.56)$ & $257.71 / 232$ \\
\hline Filament (X) & 1.59 & $(1.48,1.70)$ & 13.06 & $(11.60,14.50)$ & & $\ldots$ & & $\ldots$ & & $\ldots$ & & $\ldots$ & 2.66 & $(2.40,2.73)$ & 9.24 & $(8.51,12.29)$ & $727.95 / 675$ \\
\hline Filament (S) & 0.88 & $(0.65,1.29)$ & 12.87 & $(4.30,61.33)$ & & $\ldots$ & & $\ldots$ & & $\cdots$ & & $\ldots$ & 2.18 & $(2.12,2.27)$ & 12.87 & $(10.91,13.85)$ & $548.85 / 400$ \\
\hline \multicolumn{18}{|c|}{ (d) C14-SWCX foreground model, Anders \& Grevesse (1989) abundances } \\
\hline G048+37(X) & & $\ldots$ & & $\cdots$ & 0.00 & $(0.00,5.57)$ & 7.28 & $(6.64,8.22)$ & 5.08 & $(4.79,5.62)$ & 0.07 & $(0.00,0.34)$ & 2.23 & $(2.20,2.27)$ & 7.72 & $(7.06,8.46)$ & $2058.12 / 1822$ \\
\hline MBM 12 (S) & & $\ldots$ & & $\ldots$ & 12.73 & $(4.11,21.37)$ & 3.93 & $(3.19,4.31)$ & 2.66 & $(2.27,3.05)$ & 0.00 & $(0.00,0.10)$ & 1.71 & $(1.47,2.01)$ & 2.79 & $(1.73,4.11)$ & $401.16 / 358$ \\
\hline MBM 16 (S) & & $\ldots$ & & $\ldots$ & & ... & 13.46 & $(11.44,15.48)$ & 5.37 & $(4.43,6.16)$ & 1.01 & $(0.60,1.35)$ & & $2.10^{\mathrm{f}}$ & 0.37 & $(0.00,1.69)$ & $260.91 / 267$ \\
\hline MBM 20 (X) & & $\ldots$ & & $\ldots$ & 2.01 & $(0.37,4.46)$ & 2.60 & $(2.25,2.81)$ & 0.85 & $(0.73,1.02)$ & 0.00 & $(0.00,0.02)$ & 2.06 & $(2.04,2.09)$ & 4.45 & $(4.38,4.61)$ & $4056.98 / 3601$ \\
\hline MBM 20 (S) & & $\ldots$ & & $\ldots$ & 38.36 & $(17.25,50.01)$ & 3.34 & $(2.25,4.47)$ & 1.83 & $(1.15,2.47)$ & 0.00 & $(0.00,0.16)$ & 2.24 & $(2.10,2.35)$ & 2.94 & $(2.58,3.30)$ & $411.30 / 361$ \\
\hline $\mathrm{G} 236+38(\mathrm{~S})$ & & $\ldots$ & & $\ldots$ & 67.21 & $(0.00,145.31)$ & 17.28 & $(13.32,21.18)$ & 6.16 & $(3.59,8.92)$ & 0.00 & $(0.00,1.03)$ & 1.93 & $(1.59,2.15)$ & 6.65 & $(3.88,10.98)$ & $273.72 / 230$ \\
\hline Filament (X) & & $\ldots$ & & $\ldots$ & 1.51 & $(0.00,18.12)$ & 5.93 & $(5.16,7.31)$ & 3.75 & $(2.66,4.62)$ & 0.00 & $(0.00,0.15)$ & 2.28 & $(2.24,2.35)$ & 11.35 & $(10.67,12.32)$ & $753.62 / 673$ \\
\hline Filament (S) & & $\cdots$ & & $\cdots$ & 12.30 & $(1.29,20.80)$ & 2.85 & $(2.03,3.57)$ & 1.07 & $(0.69,1.62)$ & 0.00 & $(0.00,0.06)$ & 2.20 & $(2.17,2.26)$ & 8.23 & $(7.82,8.55)$ & $584.47 / 398$ \\
\hline \multicolumn{18}{|c|}{ (e) ACX-SWCX foreground model, Anders \& Grevesse (1989) abundances } \\
\hline G048+37 (X) & 2.17 & $(2.11,2.22)$ & 1.60 & $(1.47,1.74)$ & & $\ldots$ & & $\ldots$ & & $\ldots$ & & $\ldots$ & 1.84 & $(1.78,1.93)$ & 7.82 & $(7.27,8.78)$ & $2031.89 / 1824$ \\
\hline MBM $12(\mathrm{~S})$ & 1.69 & $(1.61,1.77)$ & 1.29 & $(1.10,1.48)$ & & $\ldots$ & & $\ldots$ & & $\ldots$ & & $\ldots$ & 1.58 & $(1.34,1.72)$ & 3.43 & $(2.54,5.71)$ & $412.79 / 360$ \\
\hline MBM 16 (S) & 1.91 & $(1.80,2.00)$ & 2.22 & $(1.89,2.64)$ & & $\ldots$ & & $\ldots$ & & $\ldots$ & & $\ldots$ & & $2.10^{\mathrm{f}}$ & 0.00 & $(0.00,0.87)$ & $257.27 / 268$ \\
\hline MBM 20 (X) & 1.18 & $(1.14,1.21)$ & 0.88 & $(0.75,1.01)$ & & $\ldots$ & & $\ldots$ & & $\ldots$ & & $\ldots$ & 1.84 & $(1.82,1.86)$ & 6.03 & $(5.84,6.12)$ & $4062.64 / 3603$ \\
\hline MBM 20 (S) & 0.83 & $(0.76,0.91)$ & 22.83 & $(12.99,37.52)$ & & $\cdots$ & & $\ldots$ & & $\ldots$ & & $\ldots$ & 2.08 & $(2.00,2.17)$ & 4.26 & $(3.82,4.70)$ & $419.86 / 363$ \\
\hline G236+38 (S) & 0.84 & $(0.78,0.91)$ & 79.57 & $(43.73,130.00)$ & & $\cdots$ & & $\ldots$ & & $\cdots$ & & $\ldots$ & 1.73 & $(1.65,1.85)$ & 13.09 & $(10.93,15.24)$ & $280.79 / 232$ \\
\hline Filament (X) & 2.10 & $(1.85,2.21)$ & 1.58 & $(1.28,1.85)$ & & $\ldots$ & & $\ldots$ & & $\ldots$ & & $\ldots$ & 2.19 & $(2.13,2.29)$ & 9.27 & $(8.26,10.54)$ & $750.33 / 675$ \\
\hline Filament (S) & 0.89 & $(0.81,1.00)$ & 9.38 & $(4.66,16.29)$ & & $\ldots$ & & $\ldots$ & & $\ldots$ & & $\ldots$ & 2.16 & $(2.11,2.20)$ & 8.99 & $\begin{array}{l}(8.55,9.42) \\
(8)\end{array}$ & $580.75 / 400$ \\
\hline
\end{tabular}


Table 2 - Continued

\begin{tabular}{|c|c|c|c|c|c|c|c|c|c|c|c|c|c|c|c|c|c|}
\hline \multirow[b]{2}{*}{$\begin{array}{c}\text { Shadow }{ }^{\mathrm{a}} \\
\text { (1) }\end{array}$} & \multicolumn{12}{|c|}{ Foreground } & \multicolumn{4}{|c|}{ Halo } & \multirow[b]{2}{*}{$\begin{array}{l}\chi^{2} / \mathrm{dof} \\
(10)\end{array}$} \\
\hline & \multicolumn{2}{|c|}{$\begin{array}{c}T_{\mathrm{fg}} \\
\left(10^{6} \mathrm{~K}\right) \\
(2)\end{array}$} & \multicolumn{2}{|c|}{$\begin{array}{c}\text { Normalization }^{\text {D }} \\
\text { (3) }\end{array}$} & \multicolumn{2}{|r|}{$\begin{array}{l}I(\mathrm{C} \mathrm{v})^{\mathrm{c}, \mathrm{e}} \\
\text { (L.U.) } \\
(4)\end{array}$} & \multicolumn{2}{|r|}{$\begin{array}{l}I(\mathrm{C} \mathrm{VI})^{\mathrm{d}, \mathrm{e}} \\
(\mathrm{L} . \mathrm{U} .) \\
(5)\end{array}$} & \multicolumn{2}{|c|}{$\begin{array}{c}I(\mathrm{O} \text { VII })^{\mathrm{C}} \\
(\mathrm{L} . \mathrm{U} .) \\
(6)\end{array}$} & \multicolumn{2}{|c|}{$\begin{array}{l}I(\mathrm{O} \text { VIII })^{\mathrm{d}} \\
(\mathrm{L} . \mathrm{U} .) \\
(7)\end{array}$} & \multicolumn{2}{|r|}{$\begin{array}{c}T_{\mathrm{h}} \\
\left(10^{6} \mathrm{~K}\right) \\
(8)\end{array}$} & \multicolumn{2}{|c|}{$\begin{array}{c}\mathcal{E}_{\mathrm{h}} \\
\left(10^{-3} \mathrm{~cm}^{-6} \mathrm{pc}\right) \\
(9)\end{array}$} & \\
\hline \multicolumn{18}{|c|}{ (f) LB foreground model, Anders \& Grevesse (1989) abundances } \\
\hline G048+37 (X) & 1.32 & $(1.28,1.37)$ & 12.31 & $(10.98,13.72)$ & & $\cdots$ & & $\cdots$ & & $\cdots$ & & $\cdots$ & 2.43 & $(2.32,2.56)$ & 5.71 & $(5.26,6.70)$ & 2029.94/1824 \\
\hline MBM $12(\mathrm{~S})$ & 1.12 & $(1.06,1.22)$ & 9.23 & $(6.98,11.50)$ & & .. & & $\ldots$ & & $\ldots$ & & $\ldots$ & 1.82 & $(1.59,2.09)$ & 2.23 & $(1.24,3.31)$ & $383.39 / 360$ \\
\hline MBM 16 (S) & 1.02 & $(0.82,1.09)$ & 29.61 & $(20.28,85.70)$ & & $\ldots$ & & $\ldots$ & & $\ldots$ & & $\ldots$ & & $2.10^{\mathrm{f}}$ & 2.55 & $(1.50,3.67)$ & $285.31 / 268$ \\
\hline MBM 20 (X) & 1.07 & $(1.05,1.09)$ & 5.03 & $(4.68,5.43)$ & & $\ldots$ & & $\ldots$ & & $\ldots$ & & $\ldots$ & 2.07 & $(2.05,2.10)$ & 4.25 & $(4.15,4.36)$ & $4052.79 / 3603$ \\
\hline MBM 20 (S) & 0.70 & $(0.64,0.95)$ & 79.18 & $(17.41,157.54)$ & & $\ldots$ & & $\ldots$ & & $\ldots$ & & $\ldots$ & 2.22 & $(2.10,2.42)$ & 3.28 & $(2.73,3.84)$ & $395.17 / 363$ \\
\hline G236+38 (S) & 1.01 & $(0.81,1.07)$ & 51.74 & $(41.60,147.78)$ & & $\ldots$ & & $\ldots$ & & $\ldots$ & & $\ldots$ & 2.15 & $(1.90,2.54)$ & 4.42 & $(3.36,7.26)$ & $265.02 / 232$ \\
\hline Filament (X) & 1.40 & $(1.34,1.54)$ & 11.43 & $(9.74,13.24)$ & & $\ldots$ & & $\ldots$ & & $\ldots$ & & $\ldots$ & 2.71 & $(2.62,2.90)$ & 6.67 & $(5.48,7.53)$ & $730.96 / 675$ \\
\hline Filament (S) & 1.11 & $(0.84,1.28)$ & 8.16 & $(5.40,19.69)$ & & $\cdots$ & & $\cdots$ & & $\cdots$ & & $\cdots$ & 2.29 & $(2.19,2.43)$ & 7.04 & $(5.82,8.32)$ & $575.47 / 400$ \\
\hline \multicolumn{18}{|c|}{ (g) C14-SWCX foreground model, Wilms et al. (2000) abundances } \\
\hline G048+37 (X) & & $\cdots$ & & $\cdots$ & 0.00 & $(5.02,5.98)$ & 5.92 & $(5.23,6.79)$ & 3.06 & $(2.74,3.95)$ & 0.00 & $(0.00,0.19)$ & 2.14 & $(2.10,2.16)$ & 14.72 & $(13.13,15.60)$ & $2012.41 / 1822$ \\
\hline MBM $12(\mathrm{~S})$ & & $\ldots$ & & $\ldots$ & 13.08 & $(4.44,21.72)$ & 3.70 & $(2.95,4.45)$ & 2.43 & $(2.00,2.85)$ & 0.00 & $(0.00,0.07)$ & 1.59 & $(1.39,1.79)$ & 4.99 & $(3.31,7.53)$ & $410.32 / 358$ \\
\hline MBM 16 (S) & & $\ldots$ & & $\ldots$ & & $\ldots$ & 13.24 & $(11.18,15.22)$ & 5.35 & $(4.31,6.05)$ & 0.77 & $(0.33,1.08)$ & & $2.10^{\mathrm{f}}$ & 0.05 & $(0.00,2.22)$ & $264.22 / 267$ \\
\hline MBM 20 (X) & & $\ldots$ & & $\ldots$ & 4.49 & $(1.93,7.04)$ & 1.29 & $(1.07,1.63)$ & 0.00 & $(0.00,0.05)$ & 0.00 & $(0.00,0.05)$ & 1.84 & $(1.81,1.86)$ & 9.67 & $(9.39,9.89)$ & $3959.80 / 3601$ \\
\hline MBM 20 (S) & & $\ldots$ & & $\ldots$ & 39.41 & $(23.15,55.67)$ & 2.68 & $(1.52,3.85)$ & 1.01 & $(0.00,1.78)$ & 0.00 & $(0.00,0.13)$ & 2.06 & $(1.76,2.21)$ & 5.53 & $(4.26,8.88)$ & $403.47 / 361$ \\
\hline G236+38 (S) & & $\ldots$ & & $\ldots$ & 84.28 & $(10.66,151.69)$ & 14.02 & $(9.80,18.14)$ & 3.50 & $(0.60,6.35)$ & 0.00 & $(0.00,0.86)$ & 1.73 & $(1.44,1.85)$ & 16.78 & $(10.22,23.83)$ & $263.32 / 230$ \\
\hline Filament (X) & & $\cdots$ & & $\ldots$ & 1.90 & $(0.00,20.33)$ & 4.78 & $(4.07,6.24)$ & 2.87 & $(0.82,3.68)$ & 0.00 & $(0.00,0.17)$ & 2.24 & $(2.21,2.35)$ & 18.02 & $(16.12,19.36)$ & $738.18 / 673$ \\
\hline Filament (S) & & $\cdots$ & & $\cdots$ & 12.13 & $(4.28,20.02)$ & 1.78 & $(1.05,2.52)$ & 0.00 & $(0.00,0.48)$ & 0.00 & $(0.00,0.06)$ & 2.15 & $(2.11,2.19)$ & 13.87 & $(12.95,14.40)$ & $553.17 / 398$ \\
\hline \multicolumn{18}{|c|}{ (h) ACX-SWCX foreground model, Wilms et al. (2000) abundances } \\
\hline G048+37 (X) & 2.24 & $(2.16,2.37)$ & 1.73 & $(1.59,2.14)$ & & $\cdots$ & & $\cdots$ & & $\cdots$ & & $\cdots$ & 1.83 & $(1.74,1.90)$ & 15.30 & $(14.04,16.76)$ & $1997.18 / 1824$ \\
\hline MBM 12 (S) & 1.68 & $(1.58,1.77)$ & 1.87 & $(1.60,2.17)$ & & $\ldots$ & & $\ldots$ & & $\cdots$ & & $\ldots$ & 1.57 & $(1.36,1.65)$ & 5.81 & $(4.36,9.00)$ & $428.47 / 360$ \\
\hline MBM 16 (S) & 1.85 & $(1.74,1.92)$ & 3.69 & $(3.13,4.04)$ & & $\ldots$ & & $\ldots$ & & $\ldots$ & & $\ldots$ & & $2.10^{\mathrm{f}}$ & 0.00 & $(0.00,1.49)$ & $265.73 / 268$ \\
\hline MBM 20 (X) & 0.92 & $(0.90,0.95)$ & 5.10 & $(4.40,6.12)$ & & $\ldots$ & & $\ldots$ & & $\ldots$ & & $\ldots$ & 1.87 & $(1.84,1.88)$ & 8.92 & $(8.75,9.12)$ & $4008.11 / 3603$ \\
\hline MBM 20 (S) & 0.80 & $(0.71,0.87)$ & 38.71 & $(21.13,71.01)$ & & $\ldots$ & & $\ldots$ & & $\ldots$ & & $\ldots$ & 1.89 & $(1.76,2.06)$ & 7.52 & $(6.14,8.97)$ & $399.95 / 363$ \\
\hline G236+38 (S) & 0.82 & $(0.75,0.89)$ & 129.10 & $(69.67,219.80)$ & & $\ldots$ & & $\ldots$ & & $\cdots$ & & $\ldots$ & 1.68 & $(1.61,1.78)$ & 22.36 & $(18.92,25.80)$ & $261.45 / 232$ \\
\hline Filament (X) & 2.08 & $(1.83,2.28)$ & 2.02 & $(1.50,2.43)$ & & $\ldots$ & & $\ldots$ & & $\ldots$ & & $\ldots$ & 2.19 & $(2.10,2.26)$ & 15.76 & $(13.96,16.98)$ & $736.11 / 675$ \\
\hline Filament (S) & 0.86 & $(0.75,0.98)$ & 13.76 & $(6.00,27.73)$ & & $\ldots$ & & $\ldots$ & & $\ldots$ & & $\ldots$ & 2.15 & $(2.11,2.20)$ & 13.65 & $(12.98,14.33)$ & $549.84 / 400$ \\
\hline \multicolumn{18}{|c|}{ (i) LB foreground model, Wilms et al. (2000) abundances } \\
\hline $\mathrm{G} 048+37(\mathrm{X})$ & 1.30 & $(1.24,1.36)$ & 15.21 & $(13.21,17.49)$ & & $\ldots$ & & $\ldots$ & & $\ldots$ & & $\ldots$ & 2.22 & $(2.20,2.33)$ & 11.97 & $(11.07,13.46)$ & $1996.52 / 1824$ \\
\hline MBM 12 (S) & 1.17 & $(1.09,1.28)$ & 12.16 & $(9.11,15.42)$ & & $\ldots$ & & $\cdots$ & & $\ldots$ & & $\ldots$ & 1.65 & $(1.41,1.94)$ & 4.02 & $(2.34,6.19)$ & $394.00 / 360$ \\
\hline MBM 16 (S) & 1.03 & $(0.86,1.13)$ & 42.95 & $(28.82,95.98)$ & & $\ldots$ & & $\ldots$ & & $\ldots$ & & $\ldots$ & & $2.10^{\mathrm{f}}$ & 2.80 & $(1.16,4.43)$ & $277.00 / 268$ \\
\hline MBM 20 (X) & 0.81 & $(0.77,0.82)$ & 16.46 & $(13.63,18.05)$ & & $\ldots$ & & $\ldots$ & & $\ldots$ & & $\ldots$ & 1.86 & $(1.83,1.88)$ & 9.07 & $(8.77,9.19)$ & $3989.33 / 3603$ \\
\hline MBM 20 (S) & 0.66 & $(0.55,0.81)$ & 157.08 & $(48.14,442.71)$ & & $\ldots$ & & $\ldots$ & & $\ldots$ & & $\ldots$ & 2.07 & $(1.88,2.20)$ & 5.61 & $(4.72,6.49)$ & $387.02 / 363$ \\
\hline G236+38 (S) & 0.91 & $(0.64,1.08)$ & 104.82 & $(58.21,691.27)$ & & $\ldots$ & & $\ldots$ & & $\cdots$ & & $\cdots$ & 1.93 & $(1.70,2.20)$ & 10.46 & $(5.42,18.03)$ & $257.36 / 232$ \\
\hline Filament (X) & 1.57 & $(1.52,1.62)$ & 13.42 & $(11.12,16.23)$ & & $\ldots$ & & $\ldots$ & & $\ldots$ & & $\ldots$ & 2.70 & $(2.59,2.82)$ & 9.97 & $(8.26,10.78)$ & $725.84 / 675$ \\
\hline Filament (S) & 0.87 & $(0.66,1.24)$ & 18.48 & $(7.11,70.11)$ & & $\ldots$ & & $\ldots$ & & $\ldots$ & & $\ldots$ & 2.20 & $(2.14,2.30)$ & 12.66 & $(10.65,13.70)$ & $550.06 / 400$ \\
\hline
\end{tabular}

Note. - Values in parentheses are the $90 \%$ confidence intervals.

a The $\mathrm{X}$ or $\mathrm{S}$ in parentheses indicates whether the shadow was observed with $X M M$-Newton or Suzaku, respectively.

$\mathrm{b}$ For the LB foreground model, this is the foreground emission measure, $\mathcal{E}_{\mathrm{fg}}$, in units of $10^{-3} \mathrm{~cm}^{-6}$ pc. For the ACX-SWCX foreground model, this is the normalization of the foreground component, in units of $10^{-8} \operatorname{arcmin}^{-2}$.

${ }^{\mathrm{c}}$ Foreground $\mathrm{K} \alpha$ intensity. We have summed the intensities of the resonance, intercombination, and forbidden lines.

d Foreground Ly $\alpha$ intensity.

e If this line lies below the energy range used for a given fit, its intensity is constrained by emission from that line spreading into the included energy range due to the finite spectral resolution, and/or by the higher-energy K lines, via the Cumbee et al. (2014) CX line ratios.

$\mathrm{f}$ Value held fixed during fitting. 